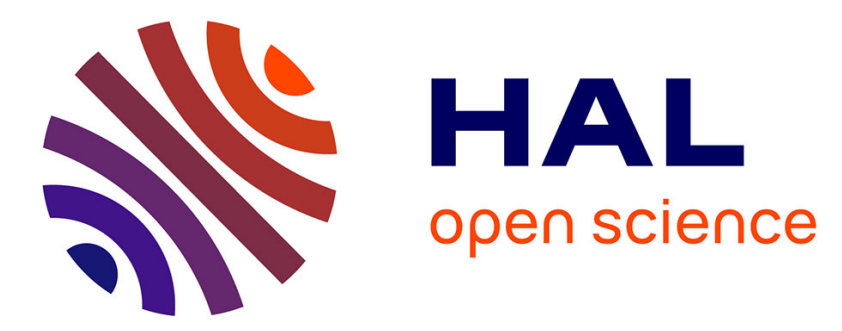

\title{
Dynamic Epistemic Logic in Update Logic
}

Guillaume Aucher

\section{To cite this version:}

Guillaume Aucher. Dynamic Epistemic Logic in Update Logic. Journal of Logic and Computation, 2016, 26 (6), pp.1913-1960. 10.1093/logcom/exw002 . hal-01476249

\section{HAL Id: hal-01476249 \\ https://inria.hal.science/hal-01476249}

Submitted on 8 Apr 2017

HAL is a multi-disciplinary open access archive for the deposit and dissemination of scientific research documents, whether they are published or not. The documents may come from teaching and research institutions in France or abroad, or from public or private research centers.
L'archive ouverte pluridisciplinaire HAL, est destinée au dépôt et à la diffusion de documents scientifiques de niveau recherche, publiés ou non, émanant des établissements d'enseignement et de recherche français ou étrangers, des laboratoires publics ou privés. 


\title{
Dynamic Epistemic Logic in Update Logic
}

\author{
Guillaume Aucher \\ University of Rennes 1 \\ INRIA \\ 263, Avenue du Général Leclerc \\ 35042 Rennes Cedex, France \\ email: guillaume.aucher@irisa.fr
}

August 12, 2016

\begin{abstract}
We show that Dynamic Epistemic Logic (DEL) is a substructural logic and that it is an extension of the update logic introduced in the companion article [12]. We identify axioms and inference rules that completely characterize the DEL product update and we provide a sequent calculus for DEL. Finally, we show that DEL with a finite number of atomic events is as expressive as epistemic logic. In parallel, we provide a sequent calculus for update logic which turns out to be a generalization of the non-associative Lambek calculus.
\end{abstract}

\section{Introduction}

Dynamic Epistemic Logic (DEL) is an influential logical framework for reasoning about the dynamics of beliefs and knowledge, which has drawn the attention of a number of researchers ever since the seminal publication of Baltag \& Al. [21]. A number of contributions have linked DEL to older and more established logical frameworks: it has been embedded into (automata) PDL [113, 107], it has been given an algebraic semantics [18, 19], and it has been related to epistemic temporal logic [105, 14] and the situation calculus [104, 112]. Despite these connections, DEL remains, arguably, a rather isolated logic in the vast realm of non-classical logics and modal logics. This is problematic if logic is to be viewed ultimately as a unified and unifying field and if we want to avoid that DEL goes on "riding off madly in all directions" (a metaphor used by van Benthem [101, 103] about logic in general). In this article we will show that DEL can be redefined naturally and meaningfully as a two-sorted substructural logic.

The well-known semantics for substructural logics is based on a ternary relation introduced by Routley and Meyer for relevance logic in the 1970's [88, 89, 90, 91]. However, the introduction of this ternary relation was originally motivated by technical reasons, and it turns out that providing a non-circular and conceptually grounded interpretation of this relation remains problematic [31]. As we shall see, the ternary semantics of DEL provides a conceptual foundation for Routley and Meyer's semantics. In fact, the dynamic interpretation induced by the DEL 
framework turns out to be not only meaningful, but also consistent with the interpretations of this ternary relation proposed in the substructural literature.

More specifically, we will show that DEL can be embedded within the substructural framework. The update logic obtained in the companion article [12] for the substructural framework will be made more specific by the addition of inference rules. This will yield a sequent calculus for DEL. Doing so, we will develop a basic correspondence theory for DEL and we will elicit a number of axioms and inference rules that play an important role in the DEL setting.

The article is structured as follows. To make it self-contained, we reintroduce a number of results and definitions of the companion article [12]. In Section 2 we recall the core of DEL viewed from a semantic perspective. In Section 3 we briefly recall elementary notions of relevance and substructural logics and we observe that the ternary relation of relevance logic can be interpreted as an update, like the DEL product update. In Section 4 we proceed further to define a generalized substructural language based on this idea. In Section 5 we recall the cut-free display calculus for update logic defined in the companion article [12] and we introduce a sequent calculus for update logic. This sequent calculus turns out to be a generalization of the non-associative Lambek calculus. In Section 6 we show that DEL can be embedded within our update logic by providing inference rules that completely characterize the DEL product update. This leads us to define a sequent calculus for DEL. Finally, in Section 7, we formally relate our DEL substructural operators to the dynamic inferences introduced in [97] and the DEL-sequents introduced in [8, 9]. We also show in this section that DEL with a finite number of atomic events is as expressive as epistemic logic. We conclude in Section 8 .

Note. Parts of Sections 2, 3.2 and 7 appear in [10] with some minor changes.

\section{Dynamic Epistemic Logic}

Dynamic epistemic logic (DEL) is a relatively recent non-classical logic [21] which extends ordinary modal epistemic logic [52] by the inclusion of event models (called $\mathcal{L}^{\mathbb{A}}$-models in this article) to describe actions, and a product update operator that defines how epistemic models are updated as the consequence of executing actions described through event models (see [20, 110, 103] for more details). Thus, the methodology of DEL is such that it splits the task of representing the agents' beliefs and knowledge into three parts: first, one represents their beliefs about an initial situation; second, one represents their beliefs about an event taking place in this situation; third, one represents the way the agents update their beliefs about the situation after (or during) the occurrence of the event. Following this methodology, we also split the exposition of the DEL framework into three sections.

Notation 1. In the rest of this article, $\mathbb{P}$ is a countable set of propositional letters called atomic facts which describe static situations, and $\mathbb{G}:=\{1, \ldots, m\}$ is a finite set of indices called agents.

\subsection{Representation of the Initial Situation: $\mathcal{L}^{\mathbb{P}}$-model}

First, we define the epistemic language $\mathcal{L}^{\mathbb{P}}$. 
Definition 1 (Language $\mathcal{L}^{\mathbb{P}}$ ). We define the language $\mathcal{L}^{\mathbb{P}}$ inductively by the following grammar in BNF, where $p$ ranges over $\mathbb{P}$ and $j$ over $\mathbb{G}$ :

$$
\mathcal{L}^{\mathbb{P}}: \varphi::=p|\neg \varphi|(\varphi \wedge \varphi)|(\varphi \vee \varphi)| \square_{j} \varphi
$$

We will use the following abbreviation: $\diamond_{j} \varphi:=\neg \square_{j} \neg \varphi$ and $\varphi \rightarrow \psi:=\neg \varphi \vee \psi$. To save parenthesis, we use the following ranking of binding strength: $\wedge, \vee, \rightarrow$ (i.e., $\wedge$ binds stronger than $\vee$ which binds stronger than $\rightarrow)$. For example, $\square_{j} \neg p \wedge q \vee r \rightarrow p$ means $\left(\left(\left(\square_{j} \neg p\right) \wedge q\right) \vee\right.$ $r) \rightarrow p$.

We denote by $\mathcal{L}_{0}^{\mathbb{P}}$ the propositional language, that is, the sublanguage of $\mathcal{L}^{\mathbb{P}}$ defined on the basis of the propositional connectives $\neg, \wedge$ and $\vee$.

A (pointed) $\mathcal{L}^{\mathbb{P}}$-model $(\mathcal{M}, w)$ represents how the actual world represented by $w$ is perceived by the agents. Atomic facts are used to state properties of this actual world.

Definition $2\left(\mathcal{L}^{\mathbb{P}}\right.$-model). A $\mathcal{L}^{\mathbb{P}}$-model is a tuple $\mathcal{M}=\left(W, R_{1}, \ldots, R_{m}, I\right)$ where:

- $W$ is a non-empty set of possible worlds,

- $R_{j} \subseteq W \times W$ is an accessibility relation on $W$, for each $j \in \mathbb{G}$,

- $I: W \rightarrow 2^{\mathbb{P}}$ is an interpretation assigning to each possible world a subset of $\mathbb{P}$.

A $\mathcal{L}^{\mathbb{P}}$-frame is a $\mathcal{L}^{\mathbb{P}}$-model without interpretation. We write $w \in \mathcal{M}$ for $w \in W$, and $(\mathcal{M}, w)$ is called a pointed $\mathcal{L}^{\mathbb{P}}$-model ( $w$ often represents the actual world). If $w, v \in W$, we write $w R_{j} v$ or $(\mathcal{M}, w) R_{j}(\mathcal{M}, v)$ for $(w, v) \in R_{j}$, and $R_{j}(w)$ denotes $\left\{v \in W \mid w R_{j} v\right\}$.

We denote by $\mathcal{E}^{\mathbb{P}}$ the set of pointed $\mathcal{L}^{\mathbb{P}}$-models and by $\mathcal{E}^{F}$ the class of pointed $\mathcal{L}^{\mathbb{P}}$-frames.

Intuitively, $w R_{j} v$ means that in world $w$ agent $j$ considers that world $v$ might correspond to the actual world. The epistemic language can be used to describe and state properties of $\mathcal{L}^{\mathbb{P}}$ models. The formula $\square_{j} \varphi$ reads as "agent $j$ believes/knows $\varphi$ " 1 Its truth conditions are defined in such a way that agent $j$ believes $\varphi$ holds in a possible world when $\varphi$ holds in all the worlds agent $j$ considers possible.

Definition 3 (Satisfaction relation $\models$ for $\mathcal{E}^{\mathbb{P}} \times \mathcal{L}^{\mathbb{P}}$ ). We define the satisfaction relation $\models \subseteq$ $\mathcal{E}^{\mathbb{P}} \times \mathcal{L}^{\mathbb{P}}$ as follows. Let $\mathcal{M}$ be a $\mathcal{L}^{\mathbb{P}}$-model, $w \in \mathcal{M}$ and $\varphi, \psi \in \mathcal{L}^{\mathbb{P}}$. The truth conditions for the atomic facts and the connectives $\neg, \wedge, \vee$ and $\square_{j}$ are defined as follows:

$$
\begin{array}{lll}
\mathcal{M}, w=p & \text { iff } & p \in I(w) \\
\mathcal{M}, w=\neg \psi & \text { iff } & \text { it is not the case that } \mathcal{M}, w \models \psi \\
\mathcal{M}, w=\varphi \wedge \psi & \text { iff } & \mathcal{M}, w \models \varphi \text { and } \mathcal{M}, w \models \psi \\
\mathcal{M}, w=\varphi \vee \psi & \text { iff } & \mathcal{M}, w \models \varphi \text { or } \mathcal{M}, w \models \psi \\
\mathcal{M}, w \models \square_{j} \varphi & \text { iff } & \text { for all } v \in R_{j}(w), \text { we have that } \mathcal{M}, v \models \varphi
\end{array}
$$

A $\mathcal{L}$-formula $\varphi$ is satisfiable if there is $(\mathcal{M}, w) \in \mathcal{E}^{\mathbb{P}}$ such that $\mathcal{M}, w \models \varphi$.

\footnotetext{
${ }^{1}$ The reading of $\square_{j} \varphi$ depends on the properties of the accessibility relation: if $R_{j}$ is reflexive (and transitive), then $\square_{j} \varphi$ typically reads "agent $j$ knows $\varphi$ ", but if $R_{j}$ is only serial (and transitive) then $\square_{j} \varphi$ reads "agent $j$ believes $\varphi$ ".
} 


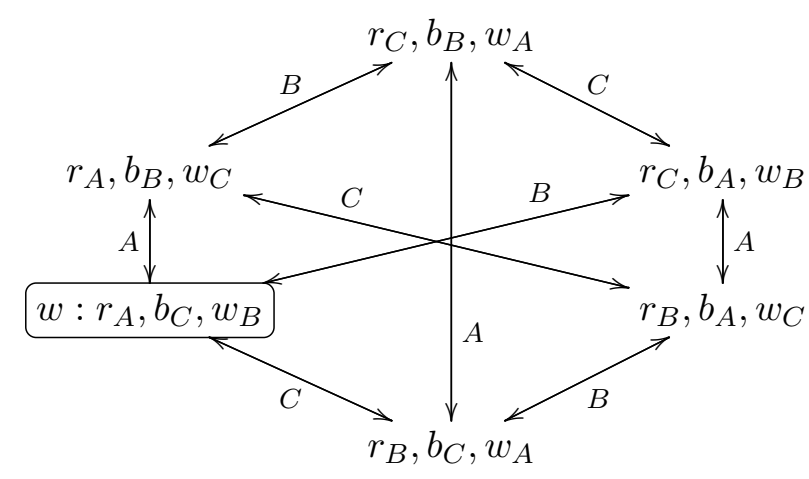

Figure 1: Cards Example

Example 1. Assume that agents A, B and C play a card game with three cards: a white one, a red one and a blue one. Each of them has a single card but they do not know the cards of the other players. At each step of the game, some of the players show their/her/his card to another player or to both other players, either privately or publicly. We want to study and represent the dynamics of the agents' beliefs in this game. The initial situation is represented by the pointed $\mathcal{L}^{\mathbb{P}}$-model $(\mathcal{M}, w)$ of Figure 1 . In this example, $\mathbb{G}:=\{A, B, C\}$ and $\mathbb{P}:=\left\{r_{j}, b_{j}, w_{j} \mid j \in \mathbb{G}\right\}$ where $r_{j}$ stands for 'agent $j$ has the red card', $b_{j}$ stands for 'agent $\mathrm{j}$ has the blue card' and $w_{j}$ stands for 'agent $j$ has the $w$ hite card'. The boxed possible world corresponds to the actual world. The propositional letters not mentioned in the possible worlds do not hold in these possible worlds. The accessibility relations are represented by arrows indexed by agents between possible worlds. Reflexive arrows are omitted in the figure, which means that for all worlds $v \in \mathcal{M}$ and all agents $j \in \mathbb{G}, v \in R_{j}(v)$. In this model, we have for example the following statement: $\mathcal{M}, w \models\left(w_{B} \wedge \neg \square_{A} w_{B}\right) \wedge \square_{C} \neg \square_{A} w_{B}$. It states that player A does not 'know' that player B has the white card and player $\mathrm{C}$ 'knows' it.

\subsection{Representation of the Event: $\mathcal{L}^{\mathbb{A}}$-model}

The language $\mathcal{L}^{\mathbb{A}}$ below was introduced by Baltag \& Al. [22]. We call the expressions $p_{\pi}$ atomic events.

Definition 4 (Language $\mathcal{L}^{\mathbb{A}}$ ). We define the language $\mathcal{L}^{\mathbb{A}}$ inductively by the following grammar in BNF, where $\pi$ ranges over $\mathcal{L}^{\mathbb{P}}$ and $j$ over $\mathbb{G}$ :

$$
\mathcal{L}^{\mathbb{A}}: \chi::=p_{\pi}|\neg \chi|(\chi \wedge \chi)|(\chi \vee \chi)| \square_{j} \chi
$$

We use the same abbreviations as in Definition 1 Like for $\mathcal{L}^{\mathbb{P}}$, we use the following ranking of binding strength: $\wedge, \vee, \rightarrow$.

A pointed $\mathcal{L}^{\mathbb{A}}$-model $(\mathcal{A}, e)$ represents how the actual event represented by $e$ is perceived by the agents. Intuitively, $f \in R_{j}^{\mathbb{A}}(e)$ means that while the possible event represented by $e$ is occurring, agent $j$ considers possible that the possible event represented by $f$ is actually occurring. 
Definition $5\left(\mathcal{L}^{\mathbb{A}}\right.$-model, [21]). A $\mathcal{L}^{\mathbb{A}}$-model is a tuple $\mathcal{A}=\left(W^{\mathbb{A}}, R_{1}^{\mathbb{A}}, \ldots, R_{m}^{\mathbb{A}}, I^{\mathbb{A}}\right)$ where:

- $W^{\mathbb{A}}$ is a non-empty set of possible events,

- $R_{j}^{\mathbb{A}} \subseteq W^{\mathbb{A}} \times W^{\mathbb{A}}$ is an accessibility relation on $W^{\mathbb{A}}$, for each $j \in \mathbb{G}$,

- $I^{\mathbb{A}}: W^{\mathbb{A}} \rightarrow \mathcal{L}^{\mathbb{P}}$ is a precondition function assigning to each possible event a formula of $\mathcal{L}^{\mathbb{P}}$.

Let $P$ be a subset of $\mathcal{L}^{\mathbb{P}}$. A $P$-complete $\mathcal{L}^{\mathbb{A}}$-model is a $\mathcal{L}^{\mathbb{A}}$-model which satisfies moreover the following condition:

- $I^{\mathbb{A}}(e) \in P$, for each $e \in W^{\mathbb{A}}$

(P-complete)

A $\mathcal{L}^{\mathbb{A}}$-frame is a $\mathcal{L}^{\mathbb{A}}$-model without precondition function $I^{\mathbb{A}}$. We abusively write $e \in \mathcal{A}$ for $e \in W^{\mathbb{A}}$, and $(\mathcal{A}, e)$ is called a pointed $\mathcal{L}^{\mathbb{A}}$-model ( $e$ often represents the actual event). We abusively write $I^{\mathbb{A}}(\mathcal{A}, e)$ for $I^{\mathbb{A}}(e)$. We denote by $\mathcal{E}^{\mathbb{A}}$ the set of pointed $\mathcal{L}^{\mathbb{A}}$-models, by $\mathcal{E}_{P}^{\mathbb{A}}$ the set of pointed $P$-complete event models and by $\mathcal{E}_{\mathbb{A}}^{F}$ the class of pointed $\mathcal{L}^{\mathbb{A}}$-frames. If $e, f \in W^{\mathbb{A}}$, we write $e R_{j}^{\mathbb{A}} f$ or $(\mathcal{A}, e) R_{j}^{\mathbb{A}}(\mathcal{A}, f)$ for $(e, f) \in R_{j}^{\mathbb{A}}$, and $R_{j}^{\mathbb{A}}(e)$ denotes $\left\{f \in W^{\mathbb{A}} \mid e R_{j}^{\mathbb{A}} f\right\}$.

The truth conditions of the language $\mathcal{L}^{\mathbb{A}}$ are almost identical to the truth conditions of the language $\mathcal{L}^{\mathbb{P}}$ :

Definition 6 (Satisfaction relation $\models$ for $\mathcal{A} \times \mathcal{L}^{\mathbb{A}}$ ). We define the satisfaction relation $\models \subseteq$ $\mathcal{A} \times \mathcal{L}^{\mathbb{P}}$ as follows. Let $\mathcal{A}$ be a $\mathcal{L}^{\mathbb{A}}$-model, $e \in \mathcal{A}$ and $\chi, \rho \in \mathcal{L}^{\mathbb{A}}$. The truth conditions for the atomic events and the connectives $\neg, \wedge, \vee$ and $\square_{j}$ are defined as follows:

$$
\begin{array}{lll}
\mathcal{A}, e \models p_{\pi} & \text { iff } & I^{\mathbb{A}}(e)=\pi \\
\mathcal{A}, e \models \neg \chi & \text { iff } & \text { it is not the case that } \mathcal{A}, e \models \chi \\
\mathcal{A}, e \models \chi \wedge \rho & \text { iff } & \mathcal{A}, e \models \chi \text { and } \mathcal{A}, e \models \rho \\
\mathcal{A}, e \models \chi \vee \rho & \text { iff } & \mathcal{A}, e \models \chi \text { or } \mathcal{A}, e \models \rho \\
\mathcal{A}, e \models \square_{j} \chi & \text { iff } & \text { for all } f \in R_{j}^{\mathbb{A}}(e), \text { we have that } \mathcal{A}, f \models \chi
\end{array}
$$

Remark 1. The current reading of $p_{\pi}$ is "an event of precondition $\pi$ is occurring". However, other truth conditions could be provided for the atomic events $p_{\pi}$. We list some of them below:

$$
\begin{array}{lll}
\mathcal{A}, e \models p_{\pi} & \text { iff } \quad \models I^{\mathbb{A}}(e) \rightarrow \pi \\
\mathcal{A}, e \models p_{\pi} & \text { iff } \quad I^{\mathbb{A}}(e) \wedge \pi \text { is satisfiable }
\end{array}
$$

The truth condition of Expression (1) states that the situation where the event $e$ occurs must necessarily satisfy the property $\pi$. The truth condition of Expression (2) turns out to be expressible in terms of Expression (1) $\left(\neg p_{\neg \pi}\right)$.

Example 2. Let us resume Example 1 and assume that players A and B show their card to each other. As it turns out, $\mathrm{C}$ noticed that A showed her card to B but did not notice that B did so to A. Players $\mathrm{A}$ and $\mathrm{B}$ know this. This event is represented in the event model $(\mathcal{A}, e)$ of Figure 2 . The boxed possible event $e$ corresponds to the actual event 'players A and B show their red and white 


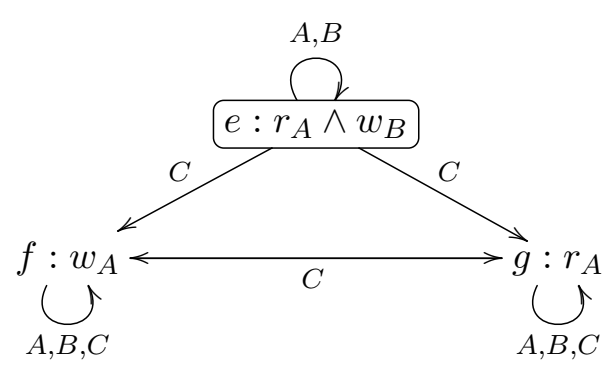

Figure 2: Players A and B show their cards to each other in front of player C

\begin{tabular}{|c|c|}
\hline$, B, C$ \\
$e: r_{A}$ \\
\hline
\end{tabular}

Figure 3: Public announcement of $r_{A}$

cards respectively to each other' (with precondition $r_{A} \wedge w_{B}$ ), $f$ stands for the event 'player A shows her white card' (with precondition $w_{A}$ ) and $g$ stands for the atomic event 'player A shows her red card' (with precondition $r_{A}$ ). The following statement holds in the example of Figure 2

$$
\begin{aligned}
\mathcal{A}, e \models p_{r_{A} \wedge w_{B}} & \wedge\left(\diamond_{A} p_{r_{A} \wedge w_{B}} \wedge \square_{A} p_{r_{A} \wedge w_{B}}\right) \wedge\left(\diamond_{B} p_{r_{A} \wedge w_{B}} \wedge \square_{B} p_{r_{A} \wedge w_{B}}\right) \\
& \wedge\left(\diamond_{C} p_{w_{A}} \wedge \diamond_{C} p_{r_{A}} \wedge \square_{C}\left(p_{w_{A}} \vee p_{r_{A}}\right)\right)
\end{aligned}
$$

It states that players A and B show their cards to each other, players A and B 'know' this and consider it possible, while player $\mathrm{C}$ considers possible that player A shows her white card and also considers possible that player A shows her red card, since he does not know her card. In fact, that is all that player $\mathrm{C}$ considers possible since he believes that either player A shows her red card or her white card ${ }^{2}$

The $\mathcal{L}^{\mathbb{A}}$-model of Figure 3 corresponds to a 'public announcement' or 'public display' of the fact that agent $\mathrm{A}$ has the red card. In particular, the following statement holds in the example of Figure 3 .

$$
\begin{aligned}
\mathcal{A}, e=p_{r_{A}} & \wedge \square_{A} p_{r_{A}} \wedge \square_{B} p_{r_{A}} \wedge \square_{C} p_{r_{A}} \\
& \wedge \square_{A} \square_{A} p_{r_{A}} \wedge \square_{A} \square_{B} p_{r_{A}} \wedge \square_{A} \square_{C} p_{r_{A}} \\
& \wedge \square_{B} \square_{A} p_{r_{A}} \wedge \square_{B} \square_{B} p_{r_{A}} \wedge \square_{B} \square_{C} p_{r_{A}} \\
& \wedge \square_{C} \square_{A} p_{r_{A}} \wedge \square_{C} \square_{B} p_{r_{A}} \wedge \square_{C} \square_{C} p_{r_{A}} \\
& \wedge \ldots
\end{aligned}
$$

It states that player A shows her red card and that players A, B and C 'know' it, that players A, B and C 'know' that each of them 'know' it, etc. in other words, there is common knowledge

\footnotetext{
${ }^{2}$ Note that accessibility relations for $C$ are no longer reflexive in $(\mathcal{A}, e)$, so we use the term belief instead of knowledge for player $C$.
} 


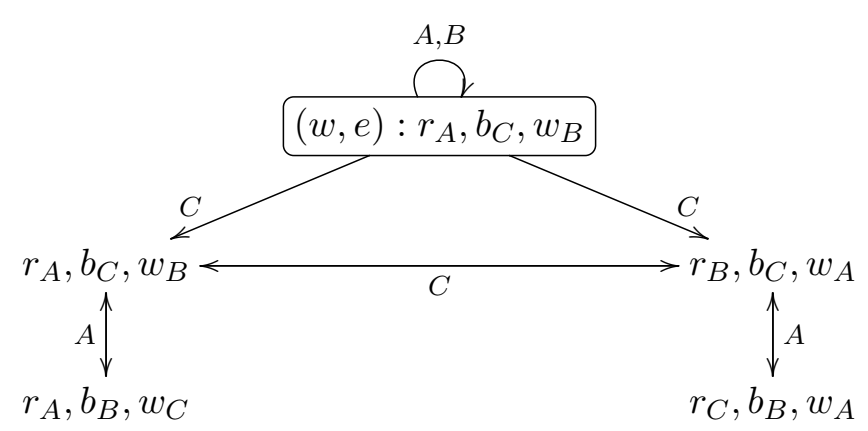

Figure 4: Situation after the Update of the Situation Represented in Figure 1 by the Event Represented in Figure 2

among players $\mathrm{A}, \mathrm{B}$ and $\mathrm{C}$ that player $\mathrm{A}$ shows her red card $\mathrm{H}^{3}$

$$
\mathcal{A}, e \models p_{r_{A}} \wedge \square_{\mathbb{G}}^{*} p_{r_{A}} .
$$

\subsection{Update of the Initial Situation by the Event: Product Update}

The DEL product update of Baltag \& Al. [21] is defined as follows. This update yields a new $\mathcal{L}^{\mathbb{P}}$ model $(\mathcal{M}, w) \otimes(\mathcal{A}, e)$ representing how the new situation which was previously represented by $(\mathcal{M}, w)$ is perceived by the agents after the occurrence of the event represented by $(\mathcal{A}, e)$.

Definition 7 (Product update). Let $(\mathcal{M}, w)=\left(W, R_{1}, \ldots, R_{m}, I, w\right)$ be a pointed $\mathcal{L}^{\mathbb{P}}$-model and let $(\mathcal{A}, e)=\left(W^{\mathbb{A}}, R_{1}^{\mathbb{A}}, \ldots, R_{m}^{\mathbb{A}}, I, e\right)$ be a pointed $\mathcal{L}^{\mathbb{A}}$-model such that $\mathcal{M}, w \models I^{\mathbb{A}}(e)$. The product update of $(\mathcal{M}, w)$ and $(\mathcal{A}, e)$ is the pointed $\mathcal{L}^{\mathbb{P}}$-model $(\mathcal{M} \otimes \mathcal{A},(w, e))=\left(W^{\otimes}, R_{1}^{\otimes}, \ldots, R_{m}^{\otimes}, I^{\otimes},(w, e)\right)$ defined as follows: for all $v \in W$ and all $f \in W^{\mathbb{A}}$,

- $W^{\otimes}=\left\{(v, f) \in W \times W^{\mathbb{A}} \mid \mathcal{M}, v \models I^{\mathbb{A}}(f)\right\}$,

- $R_{j}^{\otimes}(v, f)=\left\{(u, g) \in W^{\otimes} \mid u \in R_{j}(v)\right.$ and $\left.g \in R_{j}^{\mathbb{A}}(f)\right\}$,

- $I^{\otimes}(v, f)=I(v)$.

Example 3. As a result of the event described in Example 2, the agents update their beliefs. We get the situation represented in the $\mathcal{L}^{\mathbb{P}}$-model $(\mathcal{M}, w) \otimes(\mathcal{A}, e)$ of Figure 4 . In this model, we have for example the following statement:

$$
(\mathcal{M}, w) \otimes(\mathcal{A}, e) \models\left(w_{B} \wedge \square_{A} w_{B}\right) \wedge \square_{C} \neg \square_{A} w_{B} .
$$

It states that player A 'knows' that player B has the white card but player $\mathrm{C}$ believes that it is not the case.

\footnotetext{
${ }^{3}$ We write $\mathcal{A}, e \models \square_{\mathbb{G}}^{*} \chi$ when for all $f \in\left(\bigcup_{j \in \mathbb{G}} R_{j}^{\mathbb{A}}\right)^{*}(e)$, we have $\mathcal{A}, f \models \chi$. See for example Fagin \& Al. [38]
} for a detailed study of the operator $\square_{\mathbb{G}}^{*}$ of common knowledge. 


$\begin{array}{lll}\text { Weakening: } & \text { Contraction: } & \text { Permutation: } \\ \frac{U \vdash V}{X, U \vdash V} W_{A} & \frac{X, X, U \vdash V}{X, U \vdash V} C_{A} & \frac{U, Y, X, V \vdash W}{U, X, Y, V \vdash W} P_{A} \\ \frac{U \vdash V}{U \vdash V, X} W_{K} & \frac{U \vdash V, X, X}{U \vdash V, X} C_{K} & \frac{U \vdash V, Y, X, W}{U \vdash V, X, Y, W} P_{K}\end{array}$

Figure 5: Gentzen's Structural Rules for Classical Logic

\section{Updates as a Conceptual Foundation for Substructural Logics}

Substructural logics are a family of logics lacking some of the structural rules of classical logic. A structural rule is a rule of inference which is closed under substitution of formulas [86, Definition 2.23]. In a certain sense, a structural rule allows to manipulate the structure(s) of the sequent/consecution without altering its logical content. The structural rules for classical logic introduced by Gentzen [47] are given in Figure 5. The comma in these sequents has to be interpreted as a conjunction in an antecedent and as a disjunction in a consequent. While Weakening $\left(W_{A}, W_{K}\right)$ and Contraction $\left(C_{A}, C_{K}\right)$ are often dropped as in relevance logic and linear logic, the rule of Permutation $\left(P_{A}, P_{K}\right)$ is often preserved. When some of these rules are dropped, the comma ceases to behave as a conjunction (in the antecedent) or a disjunction (in the consequent). In that case the comma corresponds to other substructural connectives and we often introduce new punctuation marks which do not fulfill all these structural rules to deal with these new substructural connectives.

\subsection{Substructural Logics}

Our exposition of substructural logics is based on [86, 87, 37] (see also [77] for a general introduction) ${ }^{4}$ The logical framework presented in [86] is more general and studies a wide range of substructural logics: relevant logic, linear logic, Lambek calculus, arrow logic, etc. We will only introduce a fragment of this general framework in order to highlight the main new ideas. In particular, we will not consider truth sets and we will assume that our logics do not reject distribution. These other features can be added and our framework can be adapted, following the exposition of Restall [86]. We will moreover assume that we have multiple modalities (one for each agent $j \in \mathbb{G}$ ).

The semantics of substructural logics is based on the ternary relation of the frame semantics for relevant logic originally introduced by Routley and Meyer [88, 89, 90, 91]. Another semantics proposed independently by Urquhart [93, 94, 95] at about the same time will be discussed at the end of this section.

\footnotetext{
${ }^{4}$ We very slightly change the definitions of frames and models as they are defined in [86] (we give the details of these differences in the sequel). The definitions remain equivalent nevertheless.
} 
In the sequel we consider the following set of logical connectives:

$$
\text { Sub : }=\left\{\square_{j}, \diamond_{j}^{-}, \neg, \vee, \wedge, \top, \perp, \otimes, \supset, \subset, \Rightarrow \mid j \in \mathbb{G}\right\}
$$

We also define the set of connectives Sub $:=\operatorname{Sub}-\{\Rightarrow\}$ (the connective $\Rightarrow$ corresponds to the intuitionistic implication).

Definition 8 (Languages $\mathcal{L}\left(\mathbb{P}\right.$, Sub) and $\mathcal{L}\left(\mathbb{P}\right.$, Sub$\left._{-}\right)$). The language $\mathcal{L}(\mathbb{P}$, Sub $)$ is the language associated to Sub, that is, the language built compositionally from the connectives of Sub and the set of propositional letters $\mathbb{P}$. More formally, it is the set of formulas defined inductively by the following grammar in BNF, where $p$ ranges over $\mathbb{P}$ and $j$ ranges over $\mathbb{G}$ :

$$
\mathcal{L}(\mathbb{P}, \text { Sub }): \varphi::=\begin{array}{c|c|c|c|c|c|}
\top & \perp & p & (\varphi \wedge \varphi) & (\varphi \vee \varphi) & |(\varphi \Rightarrow \varphi)| \\
\square_{j} \varphi & \mid \diamond_{j}^{-} \varphi & (\varphi \otimes \varphi) & \mid(\varphi \subset \varphi) & (\varphi \supset \varphi) & \left(\varphi>{ }^{\prime}\right.
\end{array}
$$

The language $\mathcal{L}\left(\mathbb{P}\right.$, Sub $\left._{-}\right)$is the language $\mathcal{L}(\mathbb{P}$, Sub) without the (intuitionistic) connective $\Rightarrow$.

Definition 9 (Point set, accessibility relation). A point set $\mathcal{P}=(P, \sqsubseteq)$ is a non-empty set $P$ together with a partial order $\sqsubseteq$ on $P$. The set $\operatorname{Prop}(\mathcal{P})$ of propositions on $\mathcal{P}$ is the set of all subsets $X$ of $P$ which are closed upwards: that is, if $x \in X$ and $x \sqsubseteq x^{\prime}$ then $x^{\prime} \in X$. When $\sqsubseteq$ is the identity relation $=$, we say that $\mathcal{P}$ is flat. We abusively write $x \in \mathcal{P}$ for $x \in P$.

- A binary relation $R$ is a positive two-place accessibility relation on the point set $\mathcal{P}$ if, and only if, for any $x, y \in \mathcal{P}$ where $x R y$, if $x^{\prime} \sqsubseteq x$ then there is a $y^{\prime} \sqsupseteq y$ such that $x^{\prime} R y^{\prime}$. Similarly, if $x R y$ and $y \sqsubseteq y^{\prime}$ then there is some $x^{\prime} \sqsubseteq x$ such that $x^{\prime} R y^{\prime}$.

- A binary relation $R$ is a plump positive two-place accessibility relation on the point set $\mathcal{P}$ if, and only if, for any $x, y, x^{\prime}, y^{\prime} \in \mathcal{P}$, where $x R y, x^{\prime} \sqsubseteq x$ and $y \sqsubseteq y^{\prime}$ it follows that $x^{\prime} R y^{\prime}$.

- A ternary relation $\mathcal{R}$ is a three-place accessibility relation on the point set $\mathcal{P}$ if, and only if, whenever $\mathcal{R} x y z$ and $z \sqsubseteq z^{\prime}$ then there are $y^{\prime} \sqsupseteq y$ and $x^{\prime} \sqsupseteq x$ such that $\mathcal{R} x^{\prime} y^{\prime} z^{\prime}$. Similarly, if $x^{\prime} \sqsubseteq x$ then there are $y^{\prime} \sqsubseteq y$ and $z^{\prime} \sqsupseteq z$ such that $\mathcal{R} x^{\prime} y^{\prime} z^{\prime}$, and if $y^{\prime} \sqsubseteq y$ then there are $x^{\prime} \sqsubseteq x$ and $z^{\prime} \sqsupseteq z$, such that $\mathcal{R} x^{\prime} y^{\prime} z^{\prime}$.

- A ternary relation $\mathcal{R}$ is a plump three-place accessibility relation on the point set $\mathcal{P}$ if, and only if, for any $x, y, z, x^{\prime}, y^{\prime}, z^{\prime} \in \mathcal{P}$ such that $\mathcal{R} x y z$, if $x^{\prime} \sqsubseteq x, y^{\prime} \sqsubseteq y$ and $z \sqsubseteq z^{\prime}$, then $\mathcal{R} x^{\prime} y^{\prime} z^{\prime}$.

We say that $Q$ is an accessibility relation if, and only if, it is either a positive two-place or a three-place accessibility relation.

Note that plump accessibility relations are accessibility relations. The definitions of accessibility relations relate $R, \mathcal{R}$ with $\sqsubseteq$. They are set in such a way that condition (Persistence) can be lifted to arbitrary formulas of $\mathcal{L}(\mathbb{P}$, Sub $)$ and holds not only for the propositional letters of $\mathbb{P}$. 
Definition 10 (substructural model). A (multi-modal) substructural model is a tuple $\mathcal{M}=$ $\left(\mathcal{P}, R_{1}, \ldots R_{m}, \mathcal{R}, \mathcal{I}\right)$ where:

- $\mathcal{P}=(P, \sqsubseteq)$ is a point set;

- $R_{j} \subseteq \mathcal{P} \times \mathcal{P}$ is a (binary) accessibility relation on $\mathcal{P}$, for each $j \in \mathbb{G}$;

- $\mathcal{R} \subseteq \mathcal{P} \times \mathcal{P} \times \mathcal{P}$ is a (ternary) accessibility relation on $\mathcal{P}$;

- $\mathcal{I}: P \rightarrow 2^{\mathbb{P}}$ is a function called the interpretation function satisfying moreover the condition $\{x \in \mathcal{M} \mid x \in \mathcal{I}(p)\} \in \operatorname{Prop}(\mathcal{P})$, which can be reformulated as follows: for all $x, y \in P$ and all $p \in \mathbb{P}$,

$$
\text { if } p \in \mathcal{I}(x) \text { and } x \sqsubseteq y \text { then } p \in \mathcal{I}(y) .
$$

(Persistence)

We abusively write $x \in \mathcal{M}$ for $x \in \mathcal{P}$ and $(\mathcal{M}, x)$ is called a pointed substructural model. The class of all pointed substructural models is denoted $\mathcal{E}$. A (pointed) substructural frame is a (pointed) substructural model without interpretation function. The class of all pointed substructural frames is denoted $\mathcal{F}$.

Definition 11 (Evaluation relation). We define the evaluation relation $\Vdash \subseteq \mathcal{E} \times \mathcal{L}(\mathbb{P}$, Sub) as follows. Let $\mathcal{M}$ be a substructural model, $x \in \mathcal{M}$ and $\varphi, \psi \in \mathcal{L}(\mathbb{P}, \mathrm{Sub})$. The truth conditions for the atomic facts and the connectives of Sub are defined as follows:

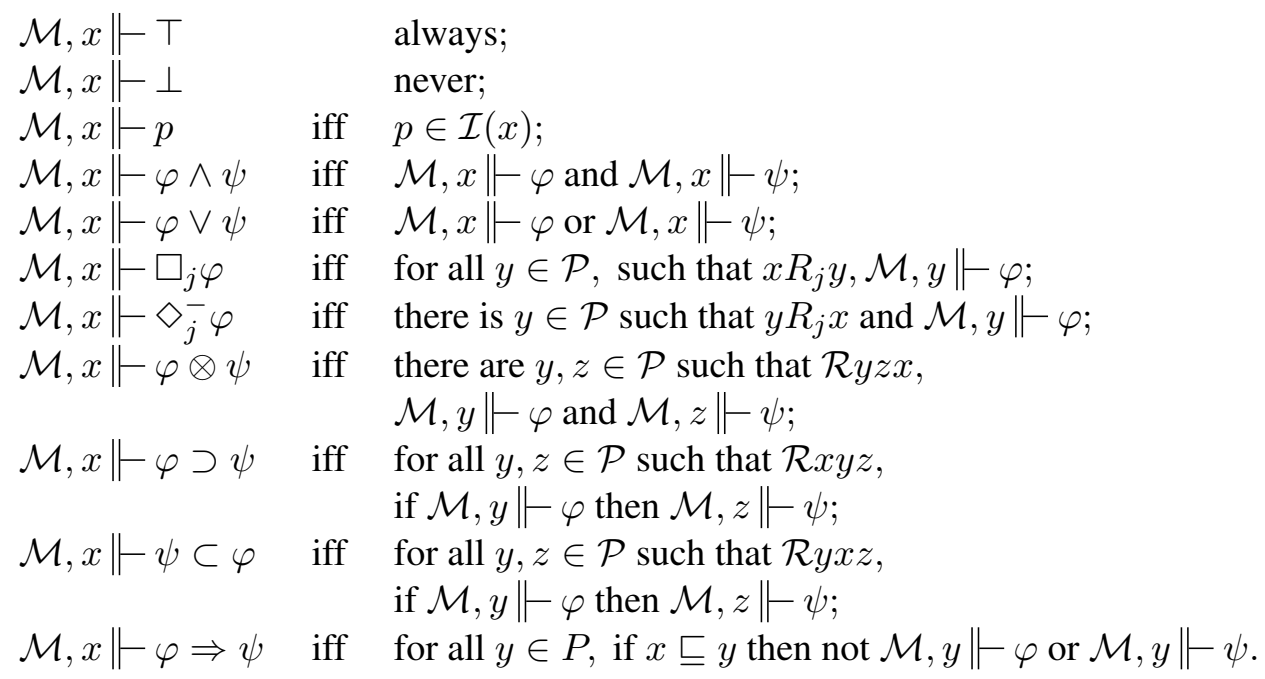

We extend these definitions to the class of pointed substructural frames. We define the evaluation relation $\Vdash \subseteq \mathcal{F} \times \mathcal{L}(\mathbb{P}$, Sub $)$ as follows. Let $(F, x)$ be a pointed frame and let $\varphi \in \mathcal{L}(\mathbb{P}$, Sub $)$. Then, we have that

$F, x \Vdash \varphi \quad$ iff $\quad$ for all interpretation functions $\mathcal{I}$ such that $(F, \mathcal{I})$ satisfies Persistence, $(F, \mathcal{I}), x \Vdash \varphi$

A substructural model stripped out from its interpretation function corresponds to a frame as defined in [86, Definition 11.8] and without truth sets. In [86], a model is a frame together with an evaluation relation. 
Urquhart's semantics. The Urquhart's semantics for relevance logic was developed independently from the Routley-Meyer's semantics in the early 1970's. We present it because its configuration is even more directly related to the DEL framework than the Routley-Meyer's semantics. An operational frame is a set of points $\mathcal{P}$ together with a function which gives us a new point from a pair of points:

$$
\sqcup: \mathcal{P} \times \mathcal{P} \rightarrow \mathcal{P}
$$

An operational model is then an operational frame together with a relation $\Vdash$ which indicates what formulas are true at what points. The truth conditions for the implication $\supset$ are defined as follows:

$$
x \Vdash \varphi \supset \psi \text { iff for each } y \text {, if } y \Vdash \varphi \text { then } x \sqcup y \Vdash \psi
$$

As one can easily notice, an operational frame is a Routley-Meyer frame where $\mathcal{R} x y z$ holds if and only if $x \sqcup y=z$. Hence, the ternary relation $\mathcal{R}$ of the Routley-Meyer semantics is a generalization of the function $\sqcup$ of the Urquhart's semantics. Because it is a relation, it allows moreover to apply $x$ to $y$ and yield either a set of outcomes or no outcome at all.

\subsection{Updates as Ternary Relations}

The ternary relation of the Routley and Meyer semantics was introduced originally for technical reasons: any 2-ary ( $n$-ary) connective of a logical language can be given a semantics by resorting to a 3-ary (resp. $n+1$-ary) relation on worlds. Subsequently, a number of philosophical interpretations of this ternary relation have been proposed and we will briefly recall some of them at the end of this section (see [31, 87, 65] for more details). However, one has to admit that providing a non-circular and conceptually grounded interpretation of this relation remains problematic [31]. In this article, we propose a new dynamic interpretation of this relation, inspired by the ternary semantics of DEL.

First, one should observe that the DEL product update $\otimes$ of Definition 7 can be seen as a partial function $\mathcal{F}_{\otimes}$ from a pair of pointed $\mathcal{L}^{\mathbb{P}}$-model and pointed $\mathcal{L}^{\mathbb{A}}$-model to another pointed $\mathcal{L}^{\mathbb{P}}$-model:

$$
\mathcal{F}_{\otimes}: \mathcal{E}^{\mathbb{P}} \times \mathcal{E}^{\mathbb{A}} \rightarrow \mathcal{E}^{\mathbb{P}}
$$

There is a formal similarity between this abstract definition of the DEL product update and the function $\sqcup$ of Expression (4) introduced by Urquhart in the early 1970s for providing a semantics to the implication of relevance logic. This similarity is not only formal but also intuitively meaningful. Indeed, the intuitive interpretation of the DEL product update operator is very similar to the intuitive interpretation of the function $\sqcup$ of Urquhart. Points are sometimes also called worlds, states, situations, set-ups, and as explained by Restall:

"We have a class of points (over which $x$ and $y$ vary), and a function $\sqcup$ which gives us new points from old. The point $x \sqcup y$ is supposed, on Urquhart's interpretation, to be the body of information given by combining $x$ with $y . "$

[87, p. 363] 
and also, keeping in mind the truth conditions for the connective $\supset$ of Expression (5):

"To be committed to $A \supset B$ is to be committed to $B$ whenever we gain the information that $A$. To put it another way, a body of information warrants $A \supset B$ if and only if whenever you update that information with new information which warrants $A$, the resulting (perhaps new) body of information warrants $B . "$ (my emphasis) [87, p. 362]

From these two quotes it is natural to interpret the DEL product update $\otimes$ of Definition 7 as a specific kind of Urquhart's function $\sqcup$ (Expression (4)). Moreover, as explained by Restall, this substructural "update" can be nonmonotonic and may correspond to some sort of revision:

"[C]ombination is sometimes nonmonotonic in a natural sense. Sometimes when a body of information is combined with another body of information, some of the original body of information might be lost. This is simplest to see in the case motivating the failure of $A \vdash B \supset A$. A body of information might tell us that $A$. However, when we combine it with something which tells us $B$, the resulting body of information might no longer warrant $A$ (as $A$ might with $B$ ). Combination might not simply result in the addition of information. It may well warrant its revision." (my emphasis) [87, p. 363]

Our dynamic interpretation of the ternary relation is consistent with the above considerations: sometimes, updating beliefs amounts to revise beliefs. As it turns out, belief revision has also been extensively studied within the DEL framework and DEL has been extended to deal with this phenomenon [5, 109, 99, 25, 26, 61, 7].

More generally, an update can be seen as a partial function $\mathcal{F}_{\otimes}$ from a pair of pointed $\mathcal{L}^{\mathbb{P}}$ model and pointed $\mathcal{L}^{\mathbb{A}}$-model to a set of pointed $\mathcal{L}^{\mathbb{P}}$-model:

$$
\mathcal{F}_{\otimes}: \mathcal{E}^{\mathbb{P}} \times \mathcal{E}^{\mathbb{A}} \rightarrow \mathcal{P}\left(\mathcal{E}^{\mathbb{P}}\right)
$$

Equivalently, an update can be seen as a ternary relation $\mathrm{UL}^{*}\left(\mathrm{C}_{\varphi}\right)$ defined on $\mathcal{E}^{\mathbb{P}} \cup \mathcal{E}^{\mathbb{A}}$ between three pointed models $\left((\mathcal{M}, w),(\mathcal{A}, e),\left(\mathcal{M}_{f}, w_{f}\right)\right)$ where $(\mathcal{M}, w)$ is a pointed $\mathcal{L}^{\mathbb{P}}$-model, $(\mathcal{A}, e)$ is a pointed $\mathcal{L}^{\mathbb{A}}$-model and $\left(\mathcal{M}_{f}, w_{f}\right)$ is another pointed $\mathcal{L}^{\mathbb{P}}$-model:

$$
\mathcal{R}_{\otimes} \subseteq \mathcal{E}^{\mathbb{P}} \times \mathcal{E}^{\mathbb{A}} \times \mathcal{E}^{\mathbb{P}}
$$

The ternary relation of Expression (8) then resembles the ternary relation of the Routley and Meyer semantics. This is not surprising since the Routley and Meyer semantics generalizes the Urquhart semantics (they are essentially the same, since as we explained it in the previous section, an operational frame is a Routley and Meyer frame where $\mathcal{R} x y z$ holds if and only if $x \sqcup y=z)$. Viewed from the perspective of DEL, the ternary relation then represents a particular sort of update. With this interpretation in mind, $\mathcal{R}_{\otimes} x y z$ reads as 'the occurrence of event $y$ in world $x$ results in the world $z$ ' and the corresponding conditional $\chi \supset \varphi$ reads as 'the occurrence in the current world of an event satisfying property $\chi$ results in a world satisfying $\varphi$ '.

The dynamic reading of the ternary relation and its corresponding conditional is very much in line with the so-called "Ramsey Test" of conditional logic. The Ramsey test can be viewed as the 
very first modern contribution to the logical study of conditionals and much of the contemporary work on conditional logic can be traced back to the famous footnote of Ramsey [83]. Roughly, it consists in defining a counterfactual conditional in terms of belief revision: an agent currently believes that $\varphi$ would be true if $\psi$ were true $($ i.e. $\psi \supset \varphi)$ if and only if he should believe $\varphi$ after learning $\psi$. A first attempt to provide truth conditions for conditionals, based on Ramsey's ideas, was proposed by Stalnaker. He defined his semantics by means of selection functions over possible worlds $f: W \times 2^{W} \rightarrow W$. As one can easily notice, Stalnaker's selection functions could also be considered from a formal point of view as a special kind of ternary relation, since a relation $\mathcal{R}_{f} \subseteq W \times 2^{W} \times W$ can be canonically associated to each selection function $f$. Moreover, like the ternary relation corresponding to a product update (Expression (8)), this ternary relation is 'two-sorted': the antecedent of a conditional takes value in a set of worlds (instead of a single world) ${ }^{5}$ So, the dynamic reading of the ternary semantics is consistent with the dynamic reading of conditionals proposed by Ramsey.

This dynamic reading was not really considered and investigated by substructural logicians when they connected the substructural ternary semantics with conditional logic [31]. On the other hand, the dynamic reading of inferences has been stressed to a large extent by van Benthem [100, 103] (we will come back to this point in Section 7), and also by Baltag \& Smets [23, 25, 26]. In particular, they distinguished dynamic belief revision from static (standard) belief revision: the latter is a revision of the agent's beliefs about the state of the world as it was before an event, and the former is a revision of the state of the world as it is after the event. Our dynamic interpretation of the ternary semantics of substructural logics is consistent with the interpretations proposed by substructural logicians. In fact, our point of view is also very much in line with the claim of Gärdenfors and Makinson [46, 63] that non-monotonic reasoning and belief revision are "two sides of the same coin": as a matter of fact, non-monotonic reasoning is a reasoning style and belief revision is a sort of update. The formal connection in this case also relies on a similar idea based on the Ramsey test.

To summarize our discussion, the DEL product update provides substructural logics with an intuitive and consistent interpretation of its ternary relation. This interpretation is consistent, in the sense that the intuitions underlying the definitions of the DEL framework are coherent with those underlying the ternary semantics of substructural logics, as witnessed by our quotes and citations from the substructural literature.

Other interpretations of the ternary relation One interpretation, due to Barwise [28] and developed by Restall [85], takes worlds to be 'sites' or 'channels', a site being possibly a channel and a channel being possibly a site. If $x, y$ and $z$ are sites, $\mathcal{R} x y z$ reads as ' $x$ is a channel between $y$ and $z$ '. Hence, if $\varphi \supset \psi$ is true at channel $x$, it means that all sites $y$ and $z$ connected by channel $x$ are such that if $\varphi$ is information available in $y$, then $\psi$ is information available in $z$ (see the paragraph "Related Proof Systems" at the end of Section 5.3 for more details). Another similar interpretation due to Mares [64] adapts Israel and Perry's theory of information [78] to the relational semantics. In this interpretation, worlds are situations in the sense of Barwise and

\footnotetext{
${ }^{5}$ Note that Burgess [34] already proposed a ternary semantics for conditionals, but his truth conditions and his interpretation of the ternary relation were quite different from ours.
} 
Perry's situation semantics [27] and pieces of information - called infons - can carry information about other infons: an infon might carry the information that a red light on a mobile phone carries the information that the battery of the mobile phone is low. In this interpretation, the ternary relation $\mathcal{R}$ represents the informational links in situations: if there is an informational link in situation $x$ that says that an infon $\sigma$ carries the information that the infon $\pi$ also holds, then if $\mathcal{R} x y z$ holds and $y$ contains the infon $\sigma$, then $z$ contains the infon $\pi$. Other interpretations of the ternary relation have been proposed by Beall \& Al. [31], with a particular focus on their relation to conditionality. For more information on this topic the reader is invited to consult [66] which covers the material briefly reviewed in this paragraph.

\section{Update Logic}

In this section we define our update logic. After introducing some mathematical definitions in Section 4.1, we motivate in Section 4.2 the introduction of three triples of logical connectives. These connectives generalize the triple $(\otimes, \supset, \subset)$ of substructural logics and will be given a cyclical semantics in Section 4.3 .

\subsection{Preliminary Definitions}

The general definitions of this section will be used in the rest of the article.

Definition 12 (Logic). A logic is a triple $L:=\left(\mathcal{L}\left(\mathbb{P}, \mathrm{C}_{\varphi}\right), E, \models\right)$ where

- $\mathcal{L}\left(\mathbb{P}, \mathrm{C}_{\varphi}\right)$ is a logical language defined as a set of well-formed expressions built from a set of logical (and structural) connectives $\mathrm{C}_{\varphi}$ and a set of propositional letters $\mathbb{P}$;

- $E$ is a class of pointed models or frames;

- $\models$ is a satisfaction relation which relates in a compositional manner elements of $\mathcal{L}\left(\mathbb{P}, \mathrm{C}_{\varphi}\right)$ to models of $E$ by means of so-called truth conditions.

Note that the above semantically-based definition of a logic is also used by French et Al. [41].

Example 4. The triples $(\mathcal{L}(\mathbb{P}$, Sub $), \mathcal{E}, \Vdash)$ and $\left(\mathcal{L}\left(\mathbb{P}\right.\right.$, Sub $\left.\left._{-}\right), \mathcal{E}, \Vdash\right)$ are logics. We list in Figure 6 logics that we deem to be 'classical'. The triple $\left(\mathcal{L}^{\mathbb{P}}, \mathcal{E}^{\mathbb{P}}, \models\right)$ is also a logic, called epistemic logic.

Definition 13 (Expressiveness). Let two logics $L=(\mathcal{L}, E, \models)$ and $\mathrm{L}^{\prime}=\left(\mathcal{L}^{\prime}, E, \models^{\prime}\right)$ be given (interpreted over the same class of models $E$ ). Let $\varphi \in \mathcal{L}$ and $\varphi^{\prime} \in \mathcal{L}^{\prime}$. We say that $\varphi$ is as expressive as $\varphi^{\prime}$ when $\{\mathcal{M} \in E \mid \mathcal{M} \models \varphi\}=\left\{\mathcal{M} \in E \mid \mathcal{M} \models \varphi^{\prime}\right\}$. We say that $L$ has at least the same expressive power as $L^{\prime}$, denoted $L \geq L^{\prime}$, when for all $\varphi^{\prime} \in \mathcal{L}^{\prime}$, there is $\varphi \in \mathcal{L}$ such that $\varphi$ is as expressive as $\varphi^{\prime}$. When $L$ has at least the same expressive power as $L^{\prime}$ and vice versa, we say that $L$ and $L^{\prime}$ have the same expressive power and we write it $L \equiv L^{\prime}$. Otherwise, $L$ is strictly more expressive than $\mathrm{L}^{\prime}$ and we write it $\mathrm{L}>\mathrm{L}^{\prime}$.

Example 5. It holds that $(\mathcal{L}(\mathbb{P}$, Sub $), \mathcal{E}, \Vdash)>\left(\mathcal{L}\left(\mathbb{P}\right.\right.$, Sub $\left.\left._{-}\right), \mathcal{E}, \Vdash\right)$. 


\begin{tabular}{|c|c|c|c|c|}
\hline \multicolumn{2}{|c|}{ Models $E$} & \multirow{2}{*}{ Connectives $\mathrm{C}_{\varphi}$} & $\operatorname{Logic}\left(\mathcal{L}\left(\mathbb{P}, \mathrm{C}_{\varphi}\right), E, \Vdash\right)$ \\
\hline$\sqsubseteq$ & $R$ & $\mathcal{R}$ & & \\
\hline$=$ & & & $\neg \wedge$ & Propositional Logic \\
$=$ & $\bullet$ & & $\neg \wedge \square_{j}$ & Modal Logic \\
$=$ & & $\bullet$ & $\otimes \supset \subset$ & Lambek Calculus \\
$=$ & $\bullet$ & $\bullet$ & $\neg \wedge \square_{j} \otimes \supset \subset$ & Modal Lambek Calculus \\
\hline
\end{tabular}

Figure 6: ‘Classical’ Logics

\subsection{Talking about Ternary Relations}

If we want to reason about updates, we must be able to express properties of updates. In other words, we need a language for talking about updates. Since we represent them by ternary relations, it seems natural to require that our language be able to express properties that relate what is true at each point of the ternary relations, that is, what is true at: 1. the initial situation (expressed by a formula $\varphi$ ), 2. the event occurring in this situation (expressed by a second formula $\chi$ ), 3. the resulting situation after the event has occurred (expressed by a third formula $\psi$ ):

$$
1, \varphi \longrightarrow 2, \chi \longrightarrow 3, \psi
$$

This leads us to the following general question: assume that we stand in one of these three time points $x$ (be it 1,2 or 3), what kind of property can we express and infer about the other time points $y$ and $z$ ? Here is a non-exhaustive list of the possible and most natural expressions that we would want to state:

(a) For all $y$, if $y$ satisfies $\varphi$ then for all $z, z$ satisfies $\psi$ : " $x \models \forall y \forall z(\varphi(y) \rightarrow \psi(z))$ ".

For example, in the initial state 1 , is it the case that any event satisfying $\chi$ will always lead to a state 3 satisfying $\psi$ ? Or, in state 3 , is it the case that before the occurrence of any event satisfying $\chi, \varphi$ held in all initial states 1 ?

(b) There exist $y$ and $z$ such that $y$ satisfies $\varphi$ and $z$ satisfies $\psi$ : " $x \models \exists y \exists z(\varphi(y) \wedge \psi(z))$ ".

For example, in state 1 , is it the case that there exists an event satisfying $\chi$ that may lead to a state where $\psi$ holds ? Or, in state 3 , is it possible that our current state might have been the result of an event satisfying $\chi$ in an initial state where $\varphi$ held?

(c) For all $y$ satisfying $\varphi$, there exists $z$ satisfying $\psi$ : " $x \models \forall y \exists z(\varphi(y) \rightarrow \psi(z))$ ".

For example, in state 1 , is it the case that any events satisfying $\chi$ may lead possibly to a state where $\psi$ holds ? Or, in state 3, is it the case that an event satisfying $\chi$ might have occurred so that any former situation before this event satisfied $\varphi$ ?

This list of expressions is obviously non-exhaustive. Providing formal tools that answer these kinds of questions leads to applications in artificial intelligence and theoretical computer 
science, and as it turns out, some of these questions have already been addressed in DEL and other logical formalisms (see Section 7 for more details and examples). Typically, most of the works about conditionals and belief dynamics deal with the first kind of statements (a) or (b). In fact, the conditionals $\supset$ and $\subset$ of substructural and relevance logics of the previous section are of the form (a), whereas the substructural connective $\otimes$ is of the form (b). The language that we will define will only deal with the first two kinds of expressions (a) and (b) (Section 4.3). This language is intended to capture the various conditionals and belief change operators which have been introduced in the philosophical and artificial intelligence literature.

\subsection{Syntax and Semantics of Update Logic}

We define formally formulas, structures and then consecutions (sometimes called sequents in the literature). This is an incremental definition and each of these objects is defined on the basis of the previous one. Moreover, in the sequel, we will view sets of formulas, sets of structures and sets of consecutions as logical languages.

Notation 2. In the rest of this article, we will use the following logical connectives $\operatorname{Con}_{\varphi}$ and structural connectives $\operatorname{Con}_{X}$ ( $\varphi$ will denote formulas and $X$ will denote structures):

$$
\begin{aligned}
& \operatorname{Con}_{\varphi}:=\left\{\square_{j}, \diamond_{j}^{-}, \neg, \vee, \wedge, \top, \perp, \otimes_{i}, \supset_{i}, \subset_{i} \mid i \in\{1,2,3\}, j \in \mathbb{G}\right\} \\
& \operatorname{Con}_{X}:=\left\{*, \bullet_{j},,_{i} \mid i \in\{0,1,2,3\}, j \in \mathbb{G}\right\}
\end{aligned}
$$

The connectives $\vee, \wedge, \otimes_{i}, \supset_{i}, \subset_{i},{ }_{o},,_{i}$ (where $i$ ranges over $\{1,2,3\}$ ) are binary connectives, $\square_{j}, \diamond_{j}^{-}, \neg, *, \bullet_{j}$ are unary connectives (where $j$ ranges over $\mathbb{G}$ ) and $T, \perp$ are nullary connectives. The structural connective, o will often simply be denoted, .

Definition 14 (Formula, structure and consecution).

- Let $\mathrm{C}_{\varphi} \subseteq \mathrm{Con}_{\varphi}$ be a non-empty set of logical connectives. The language associated to $C_{\varphi}$, denoted $\mathcal{L}\left(\mathbb{P}, \mathrm{C}_{\varphi}\right)$, is the language built compositionally from the connectives of $\mathrm{C}_{\varphi}$ and the set of propositional letters $\mathbb{P}$. Elements of the language $\mathcal{L}\left(\mathbb{P}, \mathrm{C}_{\varphi}\right)$ are called $\mathcal{L}\left(\mathbb{P}, C_{\varphi}\right)$-formulas and are generally denoted $\varphi, \chi, \psi, \ldots$

- Let $\mathrm{C}_{X} \subseteq \mathrm{Con}_{X}$ and $\mathrm{C}_{\varphi} \subseteq \operatorname{Con}_{\varphi}$ be non-empty sets of structural connectives and logical connectives. The set of structures associated to $C_{\varphi}$ and $C_{X}$, denoted $\mathcal{S}\left(\mathbb{P}, \mathrm{C}_{\varphi}, \mathrm{C}_{X}\right)$, is the language built compositionally from the structural connectives of $\mathrm{C}_{X}$ and the set $\mathcal{L}\left(\mathbb{P}, \mathrm{C}_{\varphi}\right)$. Elements of the language $\mathcal{S}\left(\mathbb{P}, \mathrm{C}_{\varphi}, \mathrm{C}_{X}\right)$ are called $\mathcal{S}\left(\mathbb{P}, C_{\varphi}, C_{X}\right)$-structures and are generally denoted $X, Y, Z, \ldots$

The structural connectives associated to $C_{\varphi}$, denoted $\operatorname{Struc}\left(\mathrm{C}_{\varphi}\right)$, is the set of structural connectives $\left\{*,{ }_{0}\right\}$ together with $\left\{{ }_{\mathrm{i}} \mid i \in\{1,2,3\}\right\}$ if $\mathrm{C}_{\varphi} \cap\left\{\otimes_{\mathrm{i}}, \supset_{\mathrm{i}}, \subset_{\mathrm{i}} \mid i \in\{1,2,3\}\right\} \neq$ $\emptyset$ and with $\left\{\bullet_{j}\right\}$ if $\mathrm{C}_{\varphi} \cap\left\{\square_{j}, \diamond_{j}^{-} \mid j \in \mathbb{G}\right\} \neq \emptyset$. We denote by $\mathcal{S}\left(\mathbb{P}, \mathrm{C}_{\varphi}\right)$ the set of all $\mathcal{S}\left(\mathbb{P}, \mathrm{C}_{\varphi}, \operatorname{Struc}\left(\mathrm{C}_{\varphi}\right)\right)$-structures.

- Let $\mathrm{C}_{X} \subseteq \operatorname{Con}_{X}$ and $\mathrm{C}_{\varphi} \subseteq \operatorname{Con}_{\varphi}$ be non-empty sets of structural connectives and logical connectives. A $\mathcal{S}\left(\mathbb{P}, \boldsymbol{C}_{\varphi}, C_{X}\right)$-consecution is an expression of the form $X \vdash Y$, 
$X \vdash$ or $\vdash Y$, where $X, Y \in \mathcal{S}\left(\mathbb{P}, \mathrm{C}_{\varphi}\right)$. The $\mathcal{S}\left(\mathbb{P}, \mathrm{C}_{\varphi}, \mathrm{C}_{X}\right)$-structure $X$ is called the antecedent and the $\mathcal{S}\left(\mathbb{P}, \mathrm{C}_{\varphi}, \mathrm{C}_{X}\right)$-structure $Y$ is called the consequent. Moreover, we write $X_{1}, \ldots X_{n} \vdash Y$ for $\left(\left(\ldots\left(X_{1}, \ldots, X_{n-2}\right), X_{n-1}\right), X_{n}\right) \vdash Y$. We denote by $\mathcal{C}\left(\mathrm{C}_{\varphi}\right)$ the set of all $\mathcal{S}\left(\mathbb{P}, \mathrm{C}_{\varphi}, \operatorname{Struc}\left(\mathrm{C}_{\varphi}\right)\right)$-consecutions.

The connective $\rightarrow$ (standing for the material implication) is defined by the following abbreviation: $(\varphi \rightarrow \psi):=(\neg \varphi \vee \psi)$. To avoid any ambiguity, every occurence of any binary connective is surrounded by brackets.

Example 6. If $\mathrm{C}_{\varphi}=\left\{\neg, \wedge, \otimes_{3}, \supset_{1}, \subset_{2}\right\}$, then the language $\mathcal{L}\left(\mathbb{P}, \mathrm{C}_{\varphi}\right)$ is defined by the following grammar in BNF, where $p$ ranges over $\mathbb{P}$ :

$$
\mathcal{L}\left(\mathbb{P}, \mathrm{C}_{\varphi}\right): \quad \varphi::=p|\neg \varphi|(\varphi \wedge \varphi)\left|\left(\varphi \otimes_{3} \varphi\right)\right|\left(\varphi \supset_{1} \varphi\right) \mid\left(\varphi \subset_{2} \varphi\right)
$$

Then, we have that $\operatorname{Struc}\left(\mathrm{C}_{\varphi}\right)=\left\{*,,_{0},{ }_{\mathrm{i}} \mid i \in\{1,2,3\}\right\}$. So, the language $\mathcal{S}\left(\mathbb{P}, \mathrm{C}_{\varphi}\right):=$ $\mathcal{S}\left(\mathbb{P}, \mathrm{C}_{\varphi}, \operatorname{Struc}\left(\mathrm{C}_{\varphi}\right)\right)$ is defined by the following grammar in $\mathrm{BNF}$, where $\varphi$ ranges over $\mathcal{L}\left(\mathbb{P}, \mathrm{C}_{\varphi}\right)$ and $i$ ranges over $\{1,2,3\}$ :

$$
\mathcal{S}\left(\mathbb{P}, \mathrm{C}_{\varphi}\right): X \quad:=\varphi|* X|\left(X,{ }_{0} X\right) \mid\left(X,{ }_{\mathrm{i}} X\right)
$$

Notation 3. To save parenthesis, we use the following ranking of binding strength: $\otimes_{i}, \supset_{i}$ $, \subset_{i}, \wedge, \vee, \rightarrow$ (where $i$ ranges over $\{1,2,3\}$ ). For example, $\square_{1} \neg p \wedge q \rightarrow \neg r \otimes_{3} s$ stands for $\left(\left(\square_{1}(\neg p)\right) \wedge q\right) \rightarrow\left((\neg r) \otimes_{3} s\right)$ (additional brackets have been added for the unary connectives $\square_{1}$ and $\neg$, even if they are not needed and will not appear in any formula anyway). For every binary connective $\star$, we use the following notation: $X_{1} \star \ldots \star X_{n}:=\left(\left(\ldots\left(X_{1} \star \ldots \star X_{n-2}\right) \star\right.\right.$ $\left.\left.X_{n-1}\right) \star X_{n}\right)$. For example, $\varphi_{1} \vee \ldots \vee \varphi_{n}:=\left(\left(\ldots\left(\varphi_{1} \vee \ldots \vee \varphi_{n-2}\right) \vee \varphi_{n-1}\right) \vee \varphi_{n}\right)$ and $X_{1}, \ldots, X_{n}:=\left(\left(\ldots\left(X_{1}, \ldots, X_{n-2}\right), X_{n-1}\right), X_{n}\right)$.

Definition 15 (Update logic). Let $E$ be an arbitrary set of three elements. For each $i \in\{1,2,3\}$, we define the cyclical permutations $\sigma_{i}: E^{3} \mapsto E^{3}$ as follows: for all $x, y, z \in E$,

$$
\sigma_{1}(x, y, z)=(x, y, z) \quad \sigma_{2}(x, y, z)=(z, x, y) \quad \sigma_{3}(x, y, z)=(y, z, x) .
$$

- We define the evaluation relation $\Vdash \subseteq \mathcal{E} \times \mathcal{L}\left(\mathbb{P}, \operatorname{Con}_{\varphi}\right)$ inductively as follows. Let $(\mathcal{M}, x) \in \mathcal{E}$ be a pointed substructural model and let $\varphi \in \mathcal{L}\left(\mathbb{P}, \operatorname{Con}_{\varphi}\right)$. The truth conditions for the connectives $\square_{j}, \diamond_{j}^{-}, \wedge, \vee, \perp, \top$ are defined like in Definition 11. The truth condition for the Boolean negation is defined as follows:

$$
\mathcal{M}, x \Vdash \neg \varphi \quad \text { iff } \quad \text { it is not the case that } \mathcal{M}, x \Vdash \varphi .
$$

The truth conditions for the connectives $\otimes_{\mathrm{i}}, \supset_{\mathrm{i}}, \subset_{\mathrm{i}}$ are defined as follows: for all $i \in$ $\{1,2,3\}$, we have that

$$
\begin{array}{lll}
\mathcal{M}, x \| \varphi \otimes_{\mathrm{i}} \psi \quad \text { iff } \quad & \text { there are } y, z \in \mathcal{P} \text { such that } \sigma_{i}(x, y, z) \in \mathcal{R}, \\
& \mathcal{M}, y \| \varphi \text { and } \mathcal{M}, z \| \psi ; \\
\mathcal{M}, x \| \varphi \supset_{\mathrm{i}} \psi \quad \text { iff } \quad & \text { for all } y, z \in \mathcal{P} \text { such that } \sigma_{i}(x, y, z) \in \mathcal{R}, \\
& \text { if } \mathcal{M}, y \| \varphi \text { then } \mathcal{M}, z \Vdash \psi ; \\
\mathcal{M}, x \| \varphi \subset_{\mathrm{i}} \psi \quad \text { iff } \quad & \text { for all } y, z \in \mathcal{P} \text { such that } \sigma_{i}(x, y, z) \in \mathcal{R}, \\
& \text { if } \mathcal{M}, z \| \psi \text { then } \mathcal{M}, y \Vdash \varphi .
\end{array}
$$


- We extend the scope of the evaluation relation $\|$ simultaneously in two different ways in order to also relate points to $\mathcal{S}\left(\mathbb{P}, \operatorname{Con}_{\varphi}\right)$-structures. The antecedent evaluation relation $\|^{A} \subseteq \mathcal{E} \times \mathcal{S}\left(\mathbb{P}\right.$, Con $\left._{\varphi}\right)$ is defined inductively as follows: for all $i \in\{1,2,3\}$,

$$
\begin{aligned}
& \mathcal{M}, x \| \underline{A} \varphi \quad \text { iff } \quad \mathcal{M}, x \Vdash \varphi ; \\
& \mathcal{M}, x \| \frac{A}{A} * X \quad \text { iff } \quad \text { it is not the case that } \mathcal{M}, x \|^{K} X \text {; } \\
& \mathcal{M}, x \| \underline{A} \bullet_{j} X \quad \text { iff } \quad \text { there is } y \in \mathcal{M} \text { such that } y R_{j} x \\
& \mathcal{M}, x \|^{A} X, Y \quad \text { iff } \quad \begin{array}{l}
\text { and it holds that } \mathcal{M}, y\left\|^{A}\right\|^{A} X \text { and } \mathcal{M}, x \|^{A} Y ;
\end{array} \\
& \mathcal{M}, x \|^{A} X,{ }_{\mathrm{i}} Y \quad \text { iff } \quad \text { there are } y, z \in \mathcal{M} \text { such that } \sigma_{i}(x, y, z) \in R \text {, } \\
& \mathcal{M}, y \|^{A} X \text { and } \mathcal{M}, z \|^{A} Y \text {. }
\end{aligned}
$$

The consequent evaluation relation $\|^{K} \subseteq \mathcal{E} \times \mathcal{S}\left(\mathbb{P}, \operatorname{Con}_{\varphi}\right)$ is defined inductively as follows: for all $i \in\{1,2,3\}$,

$$
\begin{array}{lll}
\mathcal{M}, x \| \frac{K}{K} \varphi & \text { iff } & \mathcal{M}, x \| \varphi ; \\
\mathcal{M}, x \| \frac{K}{K} * X & \text { iff } & \text { it is not the case that } \mathcal{M}, x \|^{A} X ; \\
\mathcal{M}, x \| \frac{K}{} \bullet_{j} X & \text { iff } & \text { for all } y \in \mathcal{M} \text { such that } x R_{j} y, \\
& & \text { it holds that } \mathcal{M}, y \|^{K} X ; \\
\mathcal{M}, x \| \frac{K}{K} X, Y & \text { iff } & \mathcal{M}, x \|^{K} X \text { or } \mathcal{M}, x \|^{K} Y ; \\
\mathcal{M}, x \|^{K} X{ }_{i} Y & \text { iff } & \text { for all } y, z \in \mathcal{M} \text { such that } \sigma_{i}(x, y, z) \in \mathcal{R}, \\
& & \mathcal{M}, y \|^{K} X \text { or } \mathcal{M}, z \|^{K} Y .
\end{array}
$$

- We extend the scope of the relation $\|$ to also relate points to $\mathcal{S}\left(\mathbb{P}, \operatorname{Con}_{\varphi}\right)$-consecutions. Depending on the form of the $\mathcal{S}\left(\mathbb{P}, \operatorname{Con}_{\varphi}\right)$-consecution, that is, whether it is of the form $X \vdash Y, \vdash Y$ or $X \vdash$, we have:

$$
\begin{array}{lll}
\mathcal{M}, x \| X \vdash Y & \text { iff } & \text { if } \mathcal{M}, x \|^{A} X, \text { then } \mathcal{M}, x \|^{K} Y ; \\
\mathcal{M}, x \| \vdash Y & \text { iff } & \mathcal{M}, x \|^{K} Y ; \\
\mathcal{M}, x \| X \vdash & \text { iff } & \text { it is not the case that } \mathcal{M}, x \|^{A} X .
\end{array}
$$

So, for all $\mathrm{C}_{\varphi} \subseteq \operatorname{Con}_{\varphi}$, the triples $\left(\mathcal{L}\left(\mathbb{P}, \mathrm{C}_{\varphi}\right), \mathcal{E}, \Vdash\right),\left(\mathcal{S}\left(\mathbb{P}, \mathrm{C}_{\varphi}\right), \mathcal{E}, \|^{A}\right),\left(\mathcal{S}\left(\mathbb{P}, \mathrm{C}_{\varphi}\right), \mathcal{E}, \|^{K}\right)$ and $\left(\mathcal{C}\left(\mathrm{C}_{\varphi}\right), \mathcal{E}, \|\right)$ are logics (as defined in Definition 12 . The triple $\left(\mathcal{L}\left(\mathbb{P}, \operatorname{Con}_{\varphi}\right), \mathcal{E}, \Vdash\right)$ is also a logic, called update logic.

Spelling out the truth conditions for the connectives $\otimes_{\mathrm{i}}, \supset_{\mathrm{i}}$ and $\subset_{\mathrm{i}}$ for $i \in\{1,2,3\}$, we obtain the expressions of Figure 7. The indices 1, 2 and 3 of our connectives indicate when formulas are evaluated. The connectives $\supset_{1}, \subset_{1}$ and $\otimes_{1}$ express properties of updates before the event, the connectives $C_{2}, \supset_{2}$ and $\otimes_{2}$ properties during the event and the connectives $\supset_{3}, \subset_{3}$ and $\otimes_{3}$ properties after the event. Typically, the formula $\varphi$ deals with the initial situation, the formula $\chi$ deals with the event and the formula $\psi$ deals with the final situation. The direction of the arrow $(\subset$ or $\supset$ ) indicates the conditional direction in which the formula should be read. For example, the formula $\psi \supset_{2} \varphi$ tells us that it should be evaluated during an event (2) and reads as "if the final situation will satisfy $\psi$ then the initial situation must necessarily satisfy $\varphi$ ", whereas $\psi \subset_{2} \varphi$ reads as "if the initial situation satisfies $\varphi$ then the final situation will 


\begin{tabular}{|c|c|}
\hline $\begin{array}{l}\mathcal{M}, x \Vdash \chi \otimes_{1} \psi \\
\mathcal{M}, x \Vdash \chi \supset_{1} \psi \\
\mathcal{M}, x \Vdash \chi \subset_{1} \psi \\
\mathcal{M}, x \Vdash \psi \otimes_{2} \varphi \\
\mathcal{M}, x \Vdash \psi \supset_{2} \varphi \\
\mathcal{M}, x \Vdash \psi \subset_{2} \varphi \\
\mathcal{M}, x \Vdash \varphi \otimes_{3} \chi \\
\mathcal{M}, x \Vdash \varphi \supset_{3} \chi \\
\mathcal{M}, x \Vdash \varphi \subset_{3} \chi\end{array}$ & 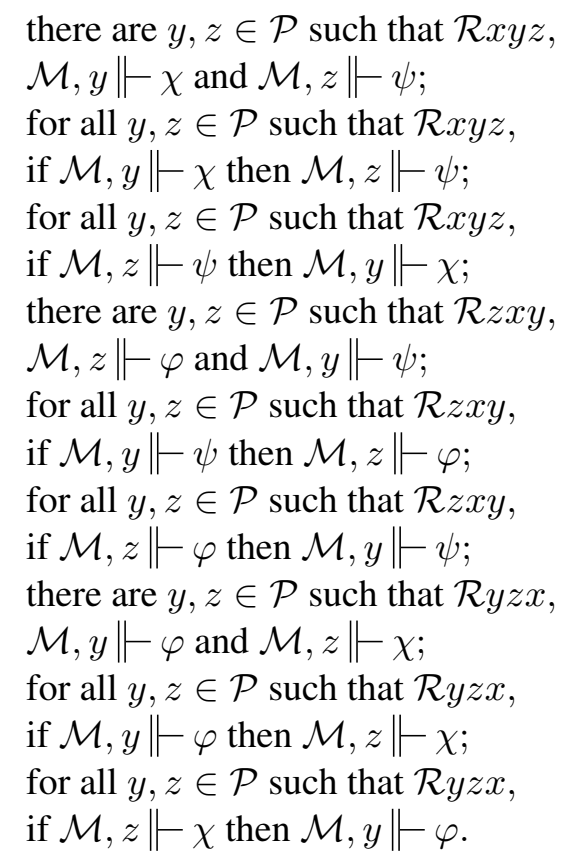 \\
\hline
\end{tabular}

Figure 7: Spelling out the Truth Conditions

necessarily satisfy $\psi$ ". The formula $\chi \supset_{1} \psi$ reads as " $\psi$ will hold after the occurrence of any events satisfying $\chi$ " and the formula $\varphi \subset_{3} \chi$ reads as " $\varphi$ held before the occurrence of any events satisfying $\chi$ ". The connectives $\otimes_{1}, \otimes_{2}, \otimes_{3}$ are of the form (b) and the connectives $\supset_{1}, \subset_{1}, \supset_{2}, \subset_{2}$ $, \supset_{3}, \subset_{3}$ are of the form (a) (see page 15 ). Note that the classical substructural connectives $\otimes, \supset$ and $\subset$ of the previous section correspond to our connectives $\otimes_{3}, \supset_{1}$ and $C_{2}$. So, our language $\mathcal{L}\left(\mathbb{P}, \operatorname{Con}_{\varphi}\right)$ extends the language $\mathcal{L}\left(\mathbb{P}\right.$, Sub $\left._{-}\right)$of substructural logics presented in Section 3.1 and the logic $\left(\mathcal{L}\left(\mathbb{P}, \operatorname{Con}_{\varphi}\right), \mathcal{E}, \Vdash\right)$ is therefore at least as expressive as $\left(\mathcal{L}\left(\mathbb{P}\right.\right.$, Sub $\left.\left._{-}\right), \mathcal{E}, \|\right)$. In fact, $\left(\mathcal{L}\left(\mathbb{P}, \operatorname{Con}_{\varphi}\right), \mathcal{E}, \Vdash\right)$ is strictly more expressive than $\left(\mathcal{L}\left(\mathbb{P}\right.\right.$, Sub$\left.\left._{-}\right), \mathcal{E}, \Vdash\right)$, as proved in [12].

\section{Proof calculi for Update Logic}

Extending Gentzen's original sequent calculi with modalities has turned out over the years to be difficult. Many of the interesting theoretical properties of sequent calculi are lost when one adds modalities (see for example Poggiolesi [80, Chapter 1] for more details). A number of methods have been proposed to overcome these difficulties: display calculi, labelled sequents, tree hypersequents (see Poggiolesi and Restall [81] for an accessible introduction to these different sorts of calculi). In this section, we provide a display calculus and a sequent calculus for our update logic. As we will see, this display calculus will be a generalization of the display calculus for modal logic introduced by Wansing [116] and the sequent calculus will be a generalization of 
the non-associative Lambek calculus NL [59, 60].

\subsection{Preliminary Definitions}

The general definitions of this section will be used in the rest of the article.

Definition 16 (Truth, validity, logical consequence). Let $\mathrm{L}=(\mathcal{L}, E, \models)$ be a logic. Let $\mathcal{M} \in E$, $\Gamma \subseteq \mathcal{L}$ and $\varphi \in \mathcal{L}$. We write $\mathcal{M} \models \Gamma$ when for all $\psi \in \Gamma$, we have $\mathcal{M} \models \psi$. Then, we say that

- $\varphi$ is true (satisfied) at $\mathcal{M}$ or $\mathcal{M}$ is a model of $\varphi$ when $\mathcal{M} \models \varphi$;

- $\varphi$ is a logical consequence of $\Gamma$, denoted $\Gamma \models\llcorner\varphi$, when for all $\mathcal{M} \in E$, if $\mathcal{M} \models \Gamma$ then $\mathcal{M} \models \varphi$;

- $\varphi$ is valid, denoted $\models\llcorner\varphi$, when for all models $\mathcal{M} \in E$, we have $\mathcal{M} \models \varphi$.

Definition 17 (Conservativity). Let $\mathrm{L}=(\mathcal{L}, E, \models)$ and $\mathrm{L}^{\prime}=\left(\mathcal{L}^{\prime}, E^{\prime}, \models^{\prime}\right)$ be two logics such that $\mathcal{L} \subseteq \mathcal{L}^{\prime}$. We say that $\mathrm{L}^{\prime}$ is a conservative extension of $\mathrm{L}$ when $\{\varphi \in \mathcal{L} \mid \models\llcorner\varphi\}=$ $\mathcal{L} \cap\left\{\varphi^{\prime} \in \mathcal{L}^{\prime} \mid \models_{\mathrm{L}^{\prime}}^{\prime} \varphi^{\prime}\right\}$.

Our definition of a proof system and of an inference rule is taken from Mendelson [67].

Definition 18 (Proof system and sequent calculus). Let $\mathrm{L}=(\mathcal{L}, E, \models)$ be a logic. A proof system $\mathcal{P}$ for $\mathcal{L}$ is a set of elements of $\mathcal{L}$ called axioms and a set of inference rules. Most often, one can effectively decide whether a given element of $\mathcal{L}$ is an axiom. To be more precise, an inference rule $R$ in $\mathcal{L}$ is a relation among elements of $\mathcal{L}$ such that there is a unique $l \in \mathbb{N}^{*}$ such that, for all $\varphi, \varphi_{1}, \ldots, \varphi_{l} \in \mathcal{L}$, one can effectively decide whether $\left(\varphi_{1}, \ldots, \varphi_{l}, \varphi\right) \in R$. The elements $\varphi_{1}, \ldots, \varphi_{l}$ are called the premises and $\varphi$ is called the conclusion and we say that $\varphi$ is a direct consequence of $\varphi_{1}, \ldots, \varphi_{l}$ by virtue of $R$. Let $\Gamma \subseteq \mathcal{L}$ and let $\varphi \in \mathcal{L}$. We say that $\varphi$ is provable (from $\Gamma$ ) in $\mathcal{P}$ or a theorem of $\mathcal{P}$, denoted $\vdash_{\mathcal{P}} \varphi\left(\right.$ resp. $\Gamma \vdash_{\mathcal{P}} \varphi$ ), when there is a proof of $\varphi$ (from $\Gamma$ ) in $\mathcal{P}$, that is, a finite sequence of formulas ending in $\varphi$ such that each of these formulas is:

1. either an instance of an axiom of $\mathcal{P}$ (or a formula of $\Gamma$ );

2. or the direct consequence of preceding formulas by virtue of an inference rule $R$.

If $\mathcal{S}$ is a set of $\mathcal{L}$-consecutions, this set $\mathcal{S}$ can be viewed as a logical language. Then, we call sequent calculus for $\mathcal{S}$ a proof system for $\mathcal{S}$.

Definition 19 (Soundness and completeness). Let $L=(\mathcal{L}, E, \models)$ be a logic. Let $\mathcal{P}$ be a proof system for $\mathcal{L}$. Then,

- $\mathcal{P}$ is sound for the logic $L$ when for all $\varphi \in \mathcal{L}$, if $\vdash_{\mathcal{P}} \varphi$, then $\models_{L} \varphi$.

- $\mathcal{P}$ is (strongly) complete for the logic $L$ when for all $\varphi \in \mathcal{L}$ (and all $\Gamma \subseteq \mathcal{L}$ ), if $\models_{\llcorner} \varphi$, then $\vdash_{\mathcal{P}} \varphi$ (resp. if $\Gamma \models_{\mathrm{L} \varphi} \varphi$, then $\Gamma \vdash_{\mathcal{P}} \varphi$ ).

Because a proof is a finite sequence of formulas, soundness for the logic $L$ coincides with 'strong' soundness for the logic $L$, i.e. for all $\varphi \in \mathcal{L}$ and all $\Gamma \subseteq \mathcal{L}$, if $\Gamma \vdash_{\mathcal{P}} \varphi$, then $\Gamma \models\llcorner\varphi$. 


\subsection{A Generalized Modal Display Calculus}

In this section, we introduce a display calculus for our update logic. It generalizes the modal display calculus of Wansing [116].

Definition 20 (Display calculus $\mathrm{UL}\left(\mathrm{C}_{\varphi}\right)$ ). Let $\mathrm{C}_{\varphi} \subseteq \operatorname{Con}_{\varphi}$. The display calculus for $\mathcal{C}\left(\boldsymbol{C}_{\varphi}\right)$, denoted $\operatorname{UL}\left(\mathrm{C}_{\varphi}\right)$, is the display calculus containing the rules of Figure 9 mentioning the logical connectives of $\mathrm{C}_{\varphi}$ and the rules of Figure 8 mentioning the structural connectives of $\operatorname{Struc}\left(\mathrm{C}_{\varphi}\right)$ (a double line means that the rule holds in both directions). When $\mathrm{C}_{\varphi}=\operatorname{Con}_{\varphi}$, the display calculus $\mathrm{UL}\left(\mathrm{C}_{\varphi}\right)$ is denoted UL. In these rules, $U$ and $V$ can be empty structures and in that case $U, X$ denotes $X$. Moreover, in rule $\otimes_{K}^{\mathrm{i}}$ (for $i \in\{1,2,3\}$ ), the consequents of the premises can also be empty and in that case the consequent of the conclusion is also empty. For better readability, the brackets for binary connectives are omitted.

A parameter in an inference rule is a structure (or formula) which is either held constant from premises to conclusion or which is introduced with no regard to its particular (formulas introduced by weakening are also parameters). A principal formulas in an inference rule is a non-parametric formula occurring in the conclusion. Congruent parameters in an inference rules are parameters that occur both in the premise(s) and the conclusion of that inference rule and that correspond to the same formula/structure. In our display calculus UL of Figures 8 and 9. principal formulas are represented by Greek formulas $\varphi, \psi$ and parameters are denoted by the Latin letters $X, Y, Z$. Congruent parameters are denoted by the same Latin letter (be it $X, Y$ or $Z)$.

Theorem 1 ([12]). Let $C_{\varphi} \subseteq \operatorname{Con}_{\varphi}$. The display calculus $\operatorname{UL}\left(C_{\varphi}\right)$ is sound and strongly complete for the logic $\left(\mathcal{C}\left(C_{\varphi}\right), \mathcal{E}, \Vdash\right)$.

Admissibility of the Cut Rule. Theorem 2 below shows that UL is a display calculus: each antecedent (consequent) part of a consecution can be 'displayed' as the sole antecedent (resp. consequent) of a structurally equivalent consecution.

Definition 21 (Antecedent and consequent part). Let $X$ be a $\mathcal{S}\left(\mathbb{P}, \operatorname{Con}_{\varphi}\right)$-structure and let $Y$ be a substructure of $X$. We say that $Y$ occurs positively in $X$ if it is in the scope of an even number of $*$. Otherwise, if $Y$ is in the scope of an odd number of $*$ in $X$, we say that $Y$ occurs negatively in $X$. If $X \vdash Y$ is a $\mathcal{S}\left(\mathbb{P}, \operatorname{Con}_{\varphi}\right)$-consecution, then $X$ is called the antecedent and $Y$ is called the consequent. Let $Z$ be a substructure of $X$ or $Y$. We say that $Z$ is an antecedent part of $X \vdash Y$ if $Z$ occurs positively in $X$ or negatively in $Y$. We say that $Z$ is a consequent part of $X \vdash Y$ if $Z$ occurs positively in $Y$ or negatively in $X$.

Theorem 2 (Display Theorem [12]). For each $\mathcal{S}\left(\mathbb{P}\right.$, Con $\left._{\varphi}\right)$-consecution $X \vdash Y$ and each antecedent part (respectively consequent part) $Z$ of $X \vdash Y$, if $X \vdash Y$ then there exists a $\mathcal{L}\left(\mathbb{P}, \operatorname{Con}_{\varphi}\right.$, Con $\left._{X}\right)$-structure $W$ such that $Z \vdash W$ (respectively $W \vdash Z$ ).

Theorem 3 (Strong cut elimination [12]). The display calculus UL is a proper display calculus. Hence, UL enjoys strong cut-elimination and therefore the cut rule is an admissible rule of UL. 


\section{Classical Rules:}

$\frac{U \vdash V}{U, X \vdash V} \mathrm{~K} \quad \frac{X, X \vdash U}{X \vdash U} \mathrm{Wl} \quad \frac{Y, X \vdash U}{X, Y \vdash U} \mathrm{Cl} \quad \frac{(X, Y), Z \vdash U}{X,(Y, Z) \vdash U} \mathrm{~B}^{c}$

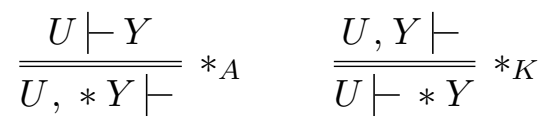

\section{Display Rules:}

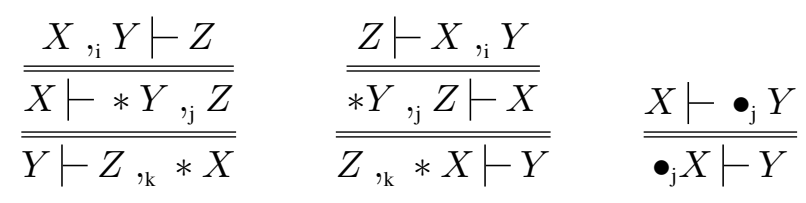

where $(i, j, k) \in\{(0,0,0),(1,2,3),(2,3,1),(3,1,2)\}$ and $j \in \mathbb{G}$

\section{Cut Rule:}

$\frac{U \vdash \varphi \quad \varphi \vdash V}{U \vdash V}$

Figure 8: Display Calculus UL: Structural Rules 
Axioms:

$\overline{p \vdash p}^{I d} \quad \overline{\perp \vdash} \perp_{A} \quad \overline{\vdash \top}^{\top} K$

Propositional Connectives:

$$
\begin{aligned}
& \frac{U \vdash * \varphi}{U \vdash \neg \varphi} \neg K \quad \frac{* \varphi \vdash U}{\neg \varphi \vdash U} \neg A \\
& \frac{U \vdash \varphi}{U \vdash \varphi \vee \psi} \vee_{K}^{1} \quad \frac{U \vdash \psi}{U \vdash \varphi \vee \psi} \vee_{K}^{2} \quad \frac{\varphi \vdash U \psi \vdash U}{\varphi \vee \psi \vdash U} \vee_{A} \\
& \frac{U \vdash \varphi \quad U \vdash \psi}{U \vdash \varphi \wedge \psi} \wedge_{K} \quad \frac{\varphi \vdash U}{\varphi \wedge \psi \vdash U} \wedge_{A}^{1} \quad \frac{\psi \vdash U}{\varphi \wedge \psi \vdash U} \wedge_{A}^{2}
\end{aligned}
$$

Modal Connectives:

$$
\begin{array}{ll}
\frac{\bullet_{\mathrm{j}} X \vdash \varphi}{X \vdash \square_{j} \varphi} \square_{K} & \frac{\varphi \vdash X}{\square_{j} \varphi \vdash \bullet_{\mathrm{j}} X} \square_{A} \\
\frac{X \vdash \varphi}{\bullet_{\mathrm{j}} X \vdash \diamond_{j}^{-} \varphi} \diamond_{K}^{-} & \frac{\varphi \vdash \bullet_{\mathrm{j}} X}{\diamond_{j}^{-} \varphi \vdash X} \diamond_{A}^{-}
\end{array}
$$

where $j \in \mathbb{G}$

Substructural Connectives:

$$
\begin{array}{ll}
\frac{X \vdash \varphi \quad Y \vdash \psi}{X,_{\mathrm{i}} Y \vdash \varphi \otimes_{\mathrm{i}} \psi} \otimes_{K}^{\mathrm{i}} & \frac{\varphi,_{\mathrm{i}} \psi \vdash U}{\varphi \otimes_{\mathrm{i}} \psi \vdash U} \otimes_{A}^{\mathrm{i}} \\
\frac{X,_{\mathrm{i}} \varphi \vdash \psi}{X \vdash \varphi \supset_{\mathrm{j}} \psi} \supset_{K}^{\mathrm{j}} & \frac{X \vdash \varphi \psi \vdash Y}{\varphi \supset_{\mathrm{j}} \psi \vdash * X,_{\mathrm{j}} Y} \supset_{A}^{\mathrm{j}} \\
\frac{\varphi,_{\mathrm{i}} X \vdash \psi}{X \vdash \psi \subset_{\mathrm{k}} \varphi} \subset_{K}^{\mathrm{k}} & \frac{\psi \vdash Y X \vdash \varphi}{\psi \subset_{\mathrm{k}} \varphi \vdash Y,_{\mathrm{k}} * X} \subset_{A}^{\mathrm{k}}
\end{array}
$$

where $(i, j, k) \in\{(1,2,3),(2,3,1),(3,1,2)\}$

Figure 9: Display Calculus UL: Logical Rules 


\begin{tabular}{|c|c|c|}
\hline$\frac{\Gamma[X] \vdash U}{\Gamma[(X, Y)] \vdash U} W_{L}$ & $\frac{\Gamma[(X, X)] \vdash U}{\Gamma[X] \vdash U} C_{L}$ & \\
\hline$\frac{U \vdash \Gamma[X]}{U \vdash \Gamma[(X, Y)]} W_{R}$ & $\frac{U \vdash \Gamma[(X, X)]}{U \vdash \Gamma[X]} C_{R}$ & \\
\hline$\frac{\Gamma[(Y, X)] \vdash U}{\Gamma[(X, Y)] \vdash U} P_{L}$ & $\frac{\Gamma[(X,(Y, Z))] \vdash U}{\Gamma[((X, Y), Z)] \vdash U} B_{L}$ & \\
\hline$\frac{U \vdash \Gamma[(Y, X)]}{U \vdash \Gamma[(X, Y)]} P_{R}$ & $\frac{U \vdash \Gamma[(X,(Y, Z))]}{U \vdash \Gamma[((X, Y), Z)]} B_{R}$ & \\
\hline$\frac{\Gamma\left[\left(\left(Z,_{2} Y\right), X\right)\right] \vdash}{\overline{\Gamma\left[\left(\left(Z,_{3} X\right), Y\right)\right] \vdash}},,_{2} /,_{3}$ & $\frac{\Gamma\left[\left(\left(X,{ }_{1} Y\right), Z\right)\right] \vdash}{\overline{\Gamma\left[\left(\left(Z,{ }_{2} Y\right), X\right)\right] \vdash}},{ }_{1} /,_{2}$ & $\frac{X \vdash \varphi \quad \Gamma[\varphi] \vdash Z}{\Gamma[X] \vdash Z}$ cut \\
\hline
\end{tabular}

Figure 10: Sequent Calculus UL*: Structural Rules

\subsection{A Generalized Lambek Calculus}

In this section, we introduce a sequent calculus for our update logic. It generalizes the nonassociative Lambek calculus [59, 60]. In fact, our sequent calculus is an intermediate between a display calculus and an LK-calculus: it has multiple structural connectives with indices (the commas and the bullets).

Definition 22 (Simple consecution). Let $\mathrm{C}_{\varphi} \subseteq \operatorname{Con}_{\varphi}$. A simple $\mathcal{S}\left(\mathbb{P}, C_{\varphi}\right)$-consecution is a $\mathcal{S}\left(\mathbb{P}, \mathrm{C}_{\varphi},\left\{{ }_{, 0},,_{1},{ }_{2}\right\}\right)$-consecution of the form $X \vdash Y, X \vdash$ or $\vdash Y$, where $X \in \mathcal{S}\left(\mathbb{P}, \mathrm{C}_{\varphi},\left\{{ }_{, 0},,_{1},,_{3}\right\}\right)$ and $Y \in \mathcal{S}\left(\mathbb{P}, \mathrm{C}_{\varphi},\left\{{ }_{0}\right\}\right)$. The set of all simple $\mathcal{S}\left(\mathbb{P}, \mathrm{C}_{\varphi}\right)$-consecutions is denoted $\mathcal{C}\left(\mathrm{C}_{\varphi}\right)^{*}$.

Let $\mathrm{C}_{\varphi} \subseteq \mathrm{Con}_{\varphi}$ and $\mathrm{C}_{X} \subseteq \mathrm{C}_{X}$. If $X$ is a $\mathcal{S}\left(\mathbb{P}, \mathrm{C}_{\varphi}, \mathrm{C}_{X}\right)$-structure, we abusively write $\varphi \in X$ when the formula $\varphi \in \mathcal{L}_{\mathrm{DEL}}$ is a substructure of $X$. The expression $\Gamma[X]$ denotes a $\mathcal{S}\left(\mathbb{P}, \mathrm{C}_{\varphi}, \mathrm{C}_{X}\right)$-structure containing as substructure the $\mathcal{S}\left(\mathbb{P}, \mathrm{C}_{\varphi}, \mathrm{C}_{X}\right)$-structure $X$, and $\Gamma[Z]$ denotes the $\mathcal{S}\left(\mathbb{P}, \mathrm{C}_{\varphi}, \mathrm{C}_{X}\right)$-structure $\Gamma[X]$ where $X$ is uniformly substituted by the structure $Z$.

Definition 23 (Sequent calculi $\mathrm{UL}^{*}\left(\mathrm{C}_{\varphi}\right)$ and $\left.\mathrm{UL}^{*}\right)$. Let $\mathrm{C}_{\varphi} \subseteq \mathrm{Con}_{\varphi}$. The sequent calculus for $\mathcal{C}\left(C_{\varphi}\right)^{*}$, denoted $\mathrm{UL}^{*}\left(\mathrm{C}_{\varphi}\right)$, is the sequent calculus of Figures 10 and 11 restricted to the logical rules for the connectives of $\mathrm{C}_{\varphi}$ (a double line means that the rule is invertible). When $\mathrm{C}_{\varphi}=\mathrm{Con}_{\varphi}$, the sequent calculus $\mathrm{UL}^{*}\left(\mathrm{C}_{\varphi}\right)$ is denoted $\mathrm{UL}^{*}$. We use the same terminology and notation as in Definition 20 .

If $\mathbf{C}_{\varphi}:=\left\{\otimes_{3}, \supset_{1}, \subset_{2}\right\}$ (as well as $\mathbf{C}_{\varphi}:=\left\{\otimes_{1}, \supset_{2}, \subset_{3}\right\}$ or $\mathbf{C}_{\varphi}:=\left\{\otimes_{2}, \supset_{3}, \subset_{1}\right\}$ ) then $\mathrm{UL}^{*}\left(\mathrm{C}_{\varphi}\right)$ is the non-associative Lambek calculus NL [59, 60]: our connective $\otimes_{3}$ corresponds to the concatenation - of the Lambek calculus, $\psi \supset_{1} \varphi$ and $\psi \subset_{2} \varphi$ correspond respectively to the connectives $\varphi / \psi$ and $\varphi \backslash \psi$ of the Lambek calculus. Moreover, if $\mathbf{C}_{\varphi}:=\left\{\square_{j}, \diamond_{j}^{-}, \otimes_{3}, \supset_{1}, \subset_{2} \mid\right.$ 
Axioms:

$\overline{p \vdash p}^{I d} \quad \overline{\perp \vdash}^{L \perp} \quad \overline{\vdash \top}^{\top} K$

Propositional Connectives:

$$
\begin{aligned}
& \frac{X, \varphi \vdash Y}{X \vdash \neg \varphi, Y} \neg R \quad \frac{X \vdash \varphi, Y}{X, \neg \varphi \vdash Y} \neg_{L} \\
& \frac{X \vdash \varphi}{X \vdash \varphi \vee \psi} \vee_{K}^{1} \quad \frac{X \vdash \psi}{X \vdash \varphi \vee \psi} \vee_{K}^{2} \quad \frac{\varphi \vdash X \quad \psi \vdash X}{\varphi \vee \psi \vdash X} \vee_{A} \\
& \frac{X \vdash \varphi \quad X \vdash \psi}{X \vdash \varphi \wedge \psi} \wedge_{K} \quad \frac{\varphi \vdash X}{\varphi \wedge \psi \vdash X} \wedge_{A}^{1} \quad \frac{\psi \vdash X}{\varphi \wedge \psi \vdash X} \wedge_{A}^{2}
\end{aligned}
$$

Modal Connectives:

$$
\begin{array}{ll}
\frac{\bullet_{\mathrm{j}} X \vdash \varphi}{X \vdash \square_{j} \varphi} \square_{K} & \frac{\Gamma[\varphi] \vdash X}{\Gamma\left[\bullet_{\mathrm{j}} \square_{j} \varphi\right] \vdash X} \square_{A} \\
\frac{X \vdash \varphi}{\bullet_{\mathrm{j}} X \vdash \diamond_{j}^{-} \varphi} \diamond_{K}^{-} & \frac{\Gamma\left[\bullet_{\mathrm{j}} \varphi\right] \vdash X}{\Gamma\left[\diamond_{j}^{-} \varphi\right] \vdash X} \diamond_{A}^{-}
\end{array}
$$

where $j \in \mathbb{G}$

Substructural Connectives:

$$
\begin{array}{ll}
\frac{X \vdash \varphi \quad Y \vdash \psi}{X,_{\mathrm{i}} Y \vdash \varphi \otimes_{\mathrm{i}} \psi} \otimes_{R}^{\mathrm{i}} & \frac{\Gamma\left[\varphi,,_{\mathrm{i}} \psi\right]-X}{\Gamma\left[\varphi \otimes_{\mathrm{i}} \psi\right] \vdash X} \otimes_{L}^{\mathrm{i}} \\
\frac{X,_{\mathrm{i}} \varphi \vdash \psi}{X \vdash \varphi \supset_{\mathrm{j}} \psi} \supset_{R}^{\mathrm{j}} & \frac{Y \vdash \psi \quad \Gamma[\varphi] \vdash X}{\Gamma\left[\left(\psi \supset_{\mathrm{j}} \varphi\right),_{\mathrm{i}} Y\right] \vdash X} \supset_{L}^{\mathrm{j}} \\
\frac{\varphi,_{\mathrm{i}} X \vdash \psi}{X \vdash \psi \subset_{\mathrm{k}} \varphi} \subset_{R}^{\mathrm{k}} & \frac{X \vdash \varphi \quad \Gamma[\psi] \vdash Y}{\Gamma\left[X,_{\mathrm{i}}\left(\psi \subset \subset_{\mathrm{k}} \varphi\right)\right] \vdash Y} \subset_{L}^{\mathrm{k}}
\end{array}
$$

where $(i, j, k) \in\{(1,2,3),(2,3,1),(3,1,2)\}$

Figure 11: Sequent Calculus UL*: Logical Rules 
$j \in \mathbb{G}\}$ then $\mathrm{UL}^{*}\left(\mathrm{C}_{\varphi}\right)$ is exactly the modal non-associative calculus $N L \diamond$ (see for example [69, Figure 5.10]). Hence, our sequent calculus $\mathrm{UL}^{*}$ is a genuine generalization of the modal non-associative Lambek calculus.

However, one should note that our update logic is not a multi-modal system (see for example [68, 69] for a definition of a multi-modal system), even if there is a similarity in the notations. The different index $i, j, k$ in the definition of a multi-modal system correspond in fact to different ternary relations $\mathcal{R}_{i}, \mathcal{R}_{j}, \mathcal{R}_{k}$.

Like the display calculus for update logic, the sequent calculus $\mathrm{UL}^{*}$ is also based on the cyclic permutations $\tau=(123)$ and $\rho=(132)$ of the set $\{1,2,3\}$ (see the companion article [12] for definitions), since $\{(1,2,3),(2,3,1),(3,1,2)\}=\{(i, \tau(i), \rho(i)) \mid i \in\{1,2,3\}\}$.

Related proof systems. The logic $\mathrm{UL}^{*}\left(\mathrm{C}_{\varphi}\right)$ with $\mathrm{C}_{\varphi}:=\left\{\wedge, \rightarrow, \otimes_{3}, \supset_{1}, \subset_{2}\right\}$ is also closely related to Barwise \& Al. [29, 30] logic of information flow. At the semantic level, this logic is also two-sorted since it contains both channels and sites. Channels correspond formally to our ternary relation and sites correspond to $\mathcal{L}^{\mathbb{P}}$-models. The different connectives introduced are counterparts of our substructural connectives, but they do not deal with propositional connectives. Moreover, instead of using in the sequents the two punctuation marks, and ${ }_{3}$ that we will use, Barwise \& Al. use two different kinds of sequents: $X \vdash Y$ and $X \mid \sim Y$. Despite these differences, the sequent calculus that they obtain for their logic of information flow is very closely related to our sequent calculus for update logic, if we restrict it to the connectives that they use.

Kurtonina [58] also introduces three fusion operators $\otimes_{1}, \otimes_{2}, \otimes_{3}$ with the same semantics as ours and provides a Hilbert style axiomatization of this language, but without modalities. However, this approach does not display the connection with the Lambek calculus and display logic as clearly as our proof systems do and it lacks all the usual interesting theoretical properties of sequent calculi (such as subformula properties, analyticity, modularity, etc. see [80, Chapter 1] for more details).

Theorem 4 (Soundness and completeness). The sequent calculus UL ${ }^{*}$ is sound and strongly complete for the logic $\left(\mathcal{C}\left(\boldsymbol{C}_{\varphi}\right)^{*}, \mathcal{E}, \|\right)$.

Proof. See Appendix.

\section{DEL as an Extension of Update Logic}

So far, we have not imposed any restrictions on our ternary relation. Therefore, there is no reason that it corresponds to the DEL product update of Definition 7 since this update was of a very specific kind: it was partial, bisimulation invariant and based on preconditions. We are going to show that DEL can be seen as an update logic if one adds specific inference rules.

Notation 4. In this section, we consider the following set Con $_{X}^{*}$ of structural connectives:

$$
\operatorname{Con}_{X}^{*}:=\left\{\bullet_{j},,_{i} \mid j \in \mathbb{G}, i \in\{0,1,2,3\}\right\}=\operatorname{Con}_{X}-\{*\} .
$$


To deal with preconditions of $\mathcal{L}^{\mathbb{A}}$-models, we introduce the following set of atomic events:

$$
\mathbb{A}:=\left\{p_{\pi} \mid \pi \in \mathcal{L}^{\mathbb{P}}\right\} .
$$

Note that $\mathbb{A}$ is countable, as is $\mathcal{L}^{\mathbb{P}}$. So, Con ${ }_{X}^{*}$ is the set of structural connectives $\operatorname{Con}_{X}$ without the Boolean structural negation $*$.

\subsection{The Logic DEL}

In this section, we redefine DEL within the substructural framework. This reformulation is completely in line with the definition of DEL as we recalled it in Section 2 .

Definition 24 (DEL substructural model). A DEL substructural model is a tuple $\mathcal{M}_{\mathrm{DEL}}=$ $\left(\mathcal{P}, \mathcal{R}_{1}, \ldots, \mathcal{R}_{m}, \mathcal{R}, \mathcal{I}\right)$ such that:

- $\mathcal{P}:=(P,=)$ is a point set such that $P \subseteq \mathcal{E}^{\mathbb{P}} \cup \mathcal{E}^{\mathbb{A}}$;

- $\mathcal{R}_{j} \subseteq \mathcal{P} \times \mathcal{P}$ is a positive two-place accessibility relation on $\mathcal{P}$ for each $j \in \mathbb{G}$ such that for all $x, y \in \mathcal{P}$, where $x=\left(\mathcal{M}_{x}, w_{x}\right)$ and $y=\left(\mathcal{M}_{y}, w_{y}\right)$ :

$$
x \in \mathcal{R}_{j}(y) \text { iff } \mathcal{M}_{x}=\mathcal{M}_{y} \text { and } w_{x} \in R_{j}\left(w_{y}\right)
$$

- $\mathcal{R}:=\left\{(x, y, z) \in P^{3} \mid x \otimes y \leftrightarrows z\right.$, with $x, z \in \mathcal{E}^{\mathbb{P}}$ and $\left.y \in \mathcal{E}^{\mathbb{A}}\right\}$ is a ternary accessibility relation on $\mathcal{P} \bigsqcup^{6}$

- $\mathcal{I}(x):=I(x)$, for all $x \in \mathcal{E}^{\mathbb{P}} \cup \mathcal{E}^{\mathbb{A}}$.

The unique DEL substructural model such that $P=\mathcal{E}^{\mathbb{P}} \cup \mathcal{E}^{\mathbb{A}}$ is called the full DEL substructural model. We abusively write $x \in \mathcal{M}$ for $x \in \mathcal{P}$ and $(\mathcal{M}, x)$ is called a pointed DEL substructural model. The class of pointed DEL substructural models is denoted $E_{\mathrm{DEL}}$.

A DEL substructural model is an update model where points are pointed $\mathcal{L}^{\mathbb{P}}$-models and pointed $\mathcal{L}^{\mathbb{A}}$-models. The ternary relation $\mathcal{R}$ is defined and motivated by the explanations of Section 3.2. Note that the accessibility relations $\mathcal{R}_{j}$ of $\mathcal{L}^{\mathbb{P}}$-models and $\mathcal{L}^{\mathbb{A}}$-models are seen in this definition as positive two-place accessibility relations $\mathcal{R}_{j}$.

Proposition 5. Any DEL substructural model is isomorphic to a substructural model.

Proof. It follows straightforwardly from the definitions.

Definition 25 (DEL language, structure and consecution).

\footnotetext{
${ }^{6} \mathrm{~A}$ bisimulation relation between pointed $\mathcal{L}^{\mathbb{P}}$-models is denoted $\leftrightarrows$. See Blackburn \& Al. [32] for more details.
} 
- The DEL language $\mathcal{L}_{D E L}$ is two-sorted and is defined by $\mathcal{L}_{\mathrm{DEL}}:=\mathcal{L}_{\mathrm{DEL}}^{\mathbb{P}} \cup \mathcal{L}_{\mathrm{DEL}}^{\mathbb{A}}$, where

$$
\begin{aligned}
& \mathcal{L}_{\mathrm{DEL}}^{\mathbb{P}}: \quad \varphi::=\begin{array}{c|c|c|c|c|c|}
\top & \perp & p & \neg \varphi & (\varphi \wedge \varphi) & (\varphi \vee \varphi) \\
\left(\chi \otimes_{1} \varphi\right) & \left(\varphi \otimes_{3} \chi\right) & \left(\chi \supset_{1} \varphi\right) & \mid\left(\varphi \supset_{3} \chi\right) & \mid\left(\chi \subset_{1} \varphi\right) & \mid\left(\varphi \subset_{3} \chi\right)
\end{array}
\end{aligned}
$$

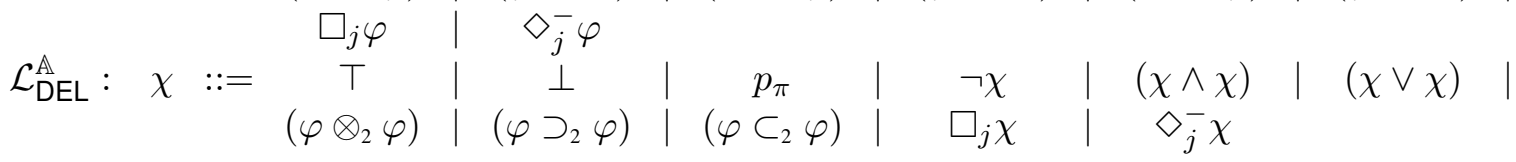

where $\pi$ ranges over $\mathcal{L}^{\mathbb{P}}$ and $j$ ranges over $\mathbb{G}$.

- The set $\mathcal{S}_{\mathrm{DEL}}$ of DEL structures is two-sorted and is defined by $\mathcal{S}_{\mathrm{DEL}}:=\mathcal{S}^{\mathbb{P}} \cup \mathcal{S}^{\mathbb{A}}$, where

$$
\begin{array}{lll|l|l|l|l|l}
\mathcal{S}^{\mathbb{P}}: X & :=\varphi & * X & \bullet_{\mathfrak{j}} X & \left(X,{ }_{0} X\right) & \left(Y,{ }_{1} X\right) & \left(X,{ }_{3} Y\right) \\
\mathcal{S}^{\mathbb{A}}: Y & :=\chi & * Y & \bullet_{j} Y & \left(Y,{ }_{0} Y\right) & \left(X,{ }_{2} X\right) &
\end{array}
$$

where $\varphi$ ranges over $\mathcal{L}_{\mathrm{DEL}}^{\mathbb{P}}, \chi$ ranges over $\mathcal{L}_{\mathrm{DEL}}^{\mathbb{A}}$ and $j$ ranges over $\mathbb{G}$.

- The set $\mathcal{C}_{\mathrm{DEL}}$ of $D E L$ consecutions is two-sorted, it is the set of simple consecutions of the form $X \vdash Y, X \vdash$ or $\vdash Y$ such that $X, Y \subseteq \mathcal{S}^{\mathbb{P}}$ or $X, Y \subseteq \mathcal{S}^{\mathbb{A}}$.

We define similarly the set of structures $\mathcal{S}_{\mathrm{DEL}}^{*}, \mathcal{S}^{\mathbb{P} *}, \mathcal{S}^{\mathbb{A} *}$ and the set of consecutions $\mathcal{C}_{\mathrm{DEL}}^{*}$ by omitting in the above definitions the structural connective $*$. Moreover, if $\mathrm{C}_{\varphi} \subseteq \mathrm{Con}_{\varphi}$, then $\mathcal{C}_{\mathrm{DEL}}\left(\mathrm{C}_{\varphi}\right)$ and $\mathcal{C}_{\mathrm{DEL}}^{*}\left(\mathrm{C}_{\varphi}\right)$ denote the consecutions of $\mathcal{C}_{\mathrm{DEL}}$ and $\mathcal{C}_{\mathrm{DEL}}^{*}$ restricted to the connectives of $\mathrm{C}_{\varphi}$.

Definition 26 (Logic DEL). We define the satisfaction relation $\Vdash \subseteq E_{\mathrm{DEL}} \times \mathcal{L}_{\mathrm{DEL}}$ as follows. The truth conditions are defined like in Definition 15, except for the case of the atomic events $p_{\pi} \in \mathbb{A}$. Let $(\mathcal{M}, x)$ be a pointed DEL substructural model. Then,

$$
\mathcal{M}, x \| p_{\pi} \quad \text { iff } \quad x \in \mathcal{E}^{\mathbb{A}} \text { and } I^{\mathbb{A}}(x)=\pi
$$

We extend the definition of the satisfaction relation to DEL structures and DEL consecutions like in Definition 15 . Hence, the triple $\left(\mathcal{L}_{\mathrm{DEL}}, E_{\mathrm{DEL}}, \|\right)$ is a logic, called the logic DEL.

\subsection{Characterizing the DEL Product Update}

The central object of our semantics is a ternary relation representing an update. Thanks to our logical language, we can now elicit a number of axioms and inference rules that define specific properties of this ternary relation. We are going to investigate which axioms and inference rules need to be added in order to recover our previous definition of the DEL product update.

\subsubsection{Correspondence Results for DEL}

Theorem 6 (DEL Correspondence Theorem). Let $A \subseteq\{$ Exclusivity), (Precondition, ,Epistemic Event $\}$, Ontic Ever (see Figure 12). Then, the sequent calculus $U L^{*}+A$ is sound and strongly complete for the logic $\left(\mathcal{L}_{D E L}, \mathcal{E}_{A}, \Vdash\right)$, where $\mathcal{E}_{A}$ is the class of substructural models satisfying the conditions of $A$. 
- $\overline{p_{\varphi}, p_{\psi} \vdash} \quad$ for all $\varphi \neq \psi$

$\forall(x, y, z) \in \mathcal{R},\left\{\pi \mid \mathcal{M}, y \Vdash p_{\pi}\right\}$ is a singleton

- $\frac{X, \pi \vdash \quad Y \vdash p_{\pi}}{X,_{3} Y \vdash} \quad$ for all $p_{\pi} \in Y$

(Precondition)

$\forall(x, y, z) \in \mathcal{R}, \forall \pi \in \mathcal{L}^{\mathbb{P}}$, if $\mathcal{M}, y \models p_{\pi}$ then $\mathcal{M}, x=\pi$

- $\frac{X \vdash p}{X,_{3} Y \vdash p} \quad \frac{X, p \vdash}{\left(X,{ }_{3} Y\right), p \vdash}$

(Epistemic Event)

$\forall(x, y, z) \in \mathcal{R}, \forall p \in \mathbb{P}, \mathcal{M}, x \models p$ iff $\mathcal{M}, z \models p$

- $\frac{X \vdash \varphi}{X,_{3} Y \vdash p} \frac{X, \varphi \vdash}{\left(X,{ }_{3} Y\right), p \vdash}$

(Ontic Event)

$\forall(x, y, z) \in \mathcal{R}, \forall p \in \mathbb{P}, \mathcal{M}, x \models \varphi$ iff $\mathcal{M}, z \models p$

- $\frac{\Phi \vdash \varphi}{\square_{j} \Phi \vdash \square_{j} \varphi}$

(Back-update)

$\forall(x, y, z) \in \mathcal{R}, \forall z^{\prime}\left(z^{\prime} \in \mathcal{R}_{j}(z) \rightarrow \exists x^{\prime} \in \mathcal{R}_{j}(x), \exists y^{\prime} \in \mathcal{R}_{j}(y),\left(x^{\prime}, y^{\prime}, z^{\prime}\right) \in \mathcal{R}\right)$

- $\frac{\varphi_{, 3} \chi \vdash \psi}{\diamond_{j}(\varphi \wedge \pi),{ }_{3} \diamond_{j}\left(\chi \wedge p_{\pi}\right) \vdash \diamond_{j} \psi}$

(Forth-update)

$\forall(x, y, z) \in \mathcal{R}, \forall \pi \in \mathcal{L}^{\mathbb{P}}, \forall x^{\prime} \in \mathcal{R}_{j}(x), \forall y^{\prime} \in \mathcal{R}_{j}(y)$,

$\left(\mathcal{M}, x^{\prime}\left\|^{A} \pi \wedge \mathcal{M}, y^{\prime}\right\|^{A} p_{\pi} \rightarrow \exists z^{\prime} \in \mathcal{R}_{j}(z),\left(x^{\prime}, y^{\prime}, z^{\prime}\right) \in \mathcal{L}_{\mathrm{DEL}}\right)$

where $p \in \mathbb{P}, \varphi, \psi \in \mathcal{L}_{\mathrm{DEL}}^{\mathbb{P}}, \chi \in \mathcal{L}_{\mathrm{DEL}}^{\mathbb{A}}, \pi \in \mathcal{L}^{\mathbb{P}}, X \in \mathcal{S}^{\mathbb{P} *}, Y \in \mathcal{S}^{\mathbb{A} *}, \Phi \in \mathcal{S}_{\left\{,_{0},,_{3}\right\}}\left(\square_{j} \Phi\right.$ is defined on page 32).

Figure 12: Inference Rules for DEL 
Proof. Soundness is proved without difficulty. So, we only prove completeness. In all cases, we are given a $\mathrm{UL}^{*}+\mathrm{R}$-consistent set $\Gamma$ (where $\mathrm{R}$ is the rule under consideration) and we have to find a substructural model $\mathcal{M}$ and $x \in \mathcal{M}$ such that (1) $\mathcal{M}, x \| \Gamma$ and (2) $\mathcal{M}$ satisfies the corresponding condition on models/frames of $R$. In all cases, we consider the canonical model for $\mathrm{UL}^{*}+\mathrm{R}$ denoted $\mathcal{M}^{c}=\left(\mathcal{P}^{c}, \mathcal{R}_{1}, \ldots, \mathcal{R}_{m}, \mathcal{R}, \mathcal{I}^{c}\right)$ as defined in the proof of Theorem 4 and we let $\Gamma^{+}$be any $U L^{*}+$ R-consistent maximal extension of $\Gamma$ obtained by the Lindenbaum Lemma of the companion article [12]. Then, by the truth Lemma of the companion article [12], $\mathcal{M}^{c}, \Gamma^{+} \Vdash \Gamma$, so step (1) is established. It remains to show for each case that $\mathcal{M}^{c}$ satisfies (2), that is, it satifies the corresponding condition on models/frames of $\mathrm{R}$.

- Rule (Back-update):

Let $\left(\Gamma, \Gamma^{\prime}, \Gamma^{\prime \prime}\right) \in \mathcal{R}$ and let $\Gamma_{1}^{\prime \prime} \in \mathcal{R}_{j}\left(\Gamma^{\prime \prime}\right)$. Let $\left(\varphi_{0}^{1}, \chi_{0}^{1}\right), \ldots,\left(\varphi_{n}^{1}, \chi_{n}^{1}\right), \ldots$ be a countable enumeration of $S:=S^{\varphi} \times S^{\chi}$, where $S^{\varphi}:=\left\{\varphi \in \mathcal{L}_{\mathrm{DEL}}^{\mathbb{P}} \mid \square_{j} \varphi \in \Gamma\right\}$ and $S^{\chi}:=\{\chi \in$ $\left.\mathcal{L}_{\mathrm{DEL}}^{\mathbb{A}} \mid \square_{j} \chi \in \Gamma^{\prime}\right\}$. We concatenate to this enumeration an arbitrary countable enumeration of $\mathcal{S}^{\mathbb{P} *} \times \mathcal{S}^{\mathbb{A} *}-S$. This yields a countable enumeration $\left(\varphi_{0}, \chi_{0}\right), \ldots,\left(\varphi_{n}, \chi_{n}\right), \ldots$ of $\mathcal{S}^{\mathbb{P} *} \times \mathcal{S}^{\mathbb{A} *}$. Then, we apply the 'pseudo'-Algorithm 1 of the companion article [12] with $\Gamma_{0}:=\emptyset$ and $\Gamma_{0}^{\prime}:=\emptyset$. This yields two sets $\Gamma_{1}$ and $\Gamma_{1}^{\prime}$. Because our enumeration starts with the formulas of $S$, we are sure to obtain that $S^{\varphi} \subseteq \Gamma_{1}$ and $S^{\chi} \subseteq \Gamma_{1}^{\prime}$. Indeed, because $\square_{j} \varphi \otimes_{3} \square_{j} \chi \in \Gamma^{\prime \prime}$ for all $\varphi \in S^{\varphi}$ and $\chi \in S^{\chi}$, we must have that $\square_{j}\left(\varphi \otimes_{3} \chi\right) \in \Gamma^{\prime \prime}$ by application of the Cut Lemma of the companion article [12] to theorem (Back-update"). Therefore, because $\Gamma_{1}^{\prime \prime} \in R_{j}\left(\Gamma^{\prime \prime}\right)$, we must have that $\varphi \otimes_{3} \chi \in \Gamma_{1}^{\prime \prime}$. This explains that during the execution of the 'pseudo'-Algorithm 1, the first conditional will always be satisfied for the pairs of formulas of $S$ since we start our enumeration with them. Therefore, we will have that $S^{\varphi} \subseteq \Gamma_{1}$ and $S^{\chi} \subseteq \Gamma_{1}^{\prime}$. So, by definition of $\mathcal{R}_{j}$ in $\mathcal{M}^{c}$, we have that $\Gamma_{1} \in R_{j}(\Gamma)$ and $\Gamma_{1}^{\prime} \in R_{j}\left(\Gamma^{\prime}\right)$. Moreover, by the same argument as the one given after the 'pseudo'-Algorithm 1, we must have that $\left(\Gamma_{1}, \Gamma_{1}^{\prime}, \Gamma_{1}^{\prime \prime}\right) \in \mathcal{R}$. This proves that $\mathcal{M}^{c}$ satisfies the condition (Back-update").

- Rule (Forth-update):

Let $\left(\Gamma, \Gamma^{\prime}, \Gamma_{f}\right) \in \mathcal{R}^{c}$ and let $\Gamma_{1} \in \mathcal{R}_{j}^{c}(\Gamma), \Gamma_{1}^{\prime} \in \mathcal{R}_{j}^{c}\left(\Gamma^{\prime}\right)$ such that $\mathcal{M}^{c}, \Gamma_{1} \|\{\pi \mid$ $\left.\mathcal{M}^{c}, \Gamma_{1}^{\prime} \Vdash p_{\pi}\right\}$. We are going to show that there is $\Gamma_{f}^{\prime} \in \mathcal{R}_{j}^{c}\left(\Gamma_{f}\right)$ such that $\left(\Gamma_{1}, \Gamma_{1}^{\prime}, \Gamma_{f}^{\prime}\right) \in$ $\mathcal{R}^{c}$. Let us consider the following set of structures: $S:=S_{1} \cup S_{2}$, where $S_{1}:=$ $\left\{\psi \mid \square_{j} \psi \in \Gamma_{f}\right\}$ and $S_{2}:=\left\{\varphi,_{3} \chi \mid \varphi \in \Gamma_{1}, \chi \in \Gamma_{1}^{\prime}\right\}$. We prove that $S$ is $\mathrm{UL}^{*}+$ (Forth-update consistent. Assume towards a contradiction that it is not. Then, there are $\psi_{1}, \ldots, \psi_{m} \in S_{1}$ and $\varphi_{1,{ }_{3}} \chi_{1}, \ldots, \varphi_{n},_{3} \chi_{n} \in S_{2}$ such that $\psi_{1}, \ldots, \psi_{m}, \varphi_{1},{ }_{3} \chi_{1}, \ldots, \varphi_{n},{ }_{3} \chi_{n} \vdash$. Then, $\varphi_{1}{ }_{3} \chi_{1}, \ldots, \varphi_{n},{ }_{3} \chi_{n} \vdash \neg \psi_{1}, \ldots, \neg \psi_{m}$, by rules $\neg R$ and $\vee_{R}$. Then, by application of the structural rules, it holds that $\varphi_{1} \wedge \ldots \wedge \varphi_{n} \wedge \pi_{{ }_{3}} \chi_{1} \wedge \ldots \wedge \chi_{n} \wedge p_{\pi} \vdash \neg \psi_{1}, \ldots, \neg \psi_{n}$. Therefore, for all $\pi \in \mathcal{L}_{\mathrm{DEL}}^{\mathbb{P}}, \varphi_{1} \wedge \ldots \wedge \varphi_{n} \wedge \pi,_{3} \chi_{1} \wedge \ldots \wedge \chi_{n} \wedge p_{\pi} \vdash \neg \psi_{1} \vee \ldots \vee \neg \psi_{n}$. So, for all $\pi \in \mathcal{L}^{\mathbb{P}}, \diamond_{j}\left(\varphi_{1} \wedge \ldots \wedge \varphi_{n} \wedge \pi\right),{ }_{3} \diamond_{j}\left(\chi_{1} \wedge \ldots \wedge \chi_{n} \wedge p_{\pi}\right) \vdash \diamond_{j}\left(\neg \psi_{1} \vee \ldots \vee \neg \psi_{n}\right)$ by application of rule Forth-update. Thus, for all $\pi \in \mathcal{L}^{\mathbb{P}}, \diamond_{j}\left(\varphi_{1} \wedge \ldots \wedge \varphi_{n} \wedge \pi\right) \otimes_{3}$ $\diamond_{j}\left(\chi_{1} \wedge \ldots \wedge \chi_{n} \wedge p_{\pi}\right) \vdash \diamond_{j}\left(\neg \psi_{1} \vee \ldots \vee \neg \psi_{n}\right)(*)$ by application of rule $\otimes_{L}^{3}$. Because $\Gamma_{1}^{\prime}$ is a maximal consistent subset, there is $p_{\pi} \in \Gamma_{1}^{\prime}$ such that $\pi \in \Gamma_{1}$ (it suffices to take $\pi=\top)$. Now, $\diamond_{j}\left(\varphi_{1} \wedge \ldots \wedge \varphi_{n} \wedge \pi\right) \in \Gamma$ and $\diamond_{j}\left(\chi_{1} \wedge \ldots \wedge \chi_{n} \wedge p_{\pi}\right) \in$ 
$\Gamma^{\prime}$. Hence, by definition of $\otimes_{3}$ in $\mathcal{M}^{c}$ and because $\left(\Gamma, \Gamma^{\prime}, \Gamma_{f}\right) \in \mathcal{R}^{c}$, we have that $\diamond_{j}\left(\varphi_{1} \wedge \ldots \wedge \varphi_{n} \wedge \pi\right) \otimes_{3} \diamond_{j}\left(\chi_{1} \wedge \ldots \wedge \chi_{n} \wedge p_{\pi}\right) \in \Gamma_{f}$. Therefore, by application of the Cut Lemma of the companion article [12] to $(*)$, we obtain that $\diamond_{j}\left(\neg \psi_{1} \vee \ldots \vee \neg \psi_{n}\right) \in \Gamma_{f}$, i.e. $\neg \square_{j}\left(\psi_{1} \wedge \ldots \wedge \psi_{n}\right) \in \Gamma_{f}$. However, by definition of $S_{1}, \square_{j} \psi_{1}, \ldots, \square_{j} \psi_{n} \in \Gamma_{f}$, and therefore $\square_{j}\left(\psi_{1} \wedge \ldots \wedge \psi_{n}\right) \in \Gamma_{f}$, which contradicts our last result. So, we reach a contradiction, therefore $S$ is $\mathrm{UL}^{*}+$ Forth-update $)$-consistent. By the Lindenbaum Lemma of the companion article [12], we can extend it to a maximal consistent subset $\Gamma_{f}^{\prime}$ of $\mathcal{L}_{\mathrm{DEL}}$. Finally, we show that $(1) \Gamma_{f}^{\prime} \in \mathcal{R}_{j}^{c}\left(\Gamma_{f}\right)$ and $(2)\left(\Gamma_{1}, \Gamma_{1}^{\prime}, \Gamma_{f}^{\prime}\right) \in \mathcal{R}^{c}$. The first item follows from the definition of $\mathcal{R}_{j}^{c}$ and the fact that $S_{1} \subseteq \Gamma_{f}^{\prime}$. The second item follows from the definition of $\mathcal{R}^{c}$ and the fact that $S_{2} \subseteq \Gamma_{f}^{\prime}$. This concludes the proof.

- Rule (Precondition):

Let $\left(\Gamma, \Gamma^{\prime}, \Gamma^{\prime \prime}\right) \in \mathcal{R}^{c}$ and let $p \in \mathbb{P}$. Assume that $\mathcal{M}^{c}, \Gamma^{\prime} \Vdash p_{\pi}$ and assume towards a contradiction that it is not the case that $\mathcal{M}^{c}, \Gamma \Vdash \pi$. Then, $\Gamma \cup\{\pi\}$ is not $U L^{*}$-consistent. So, there is $X \in \Gamma$ such that $X, \pi \vdash$. Therefore, $t_{2}(X), \pi \vdash$ and $t_{2}(X) \in \Gamma$ by Fact 2 of the companion article [12]. Moreover, $p_{\pi} \vdash p_{\pi}$. Therefore, by soundness of rule Precondition, we have that $t_{2}(X){ }_{3} \pi \vdash$. However, by definition of $\mathcal{R}^{c}$, we should have that $t_{2}(X), \pi \in \Gamma^{\prime \prime}$. This would entail that $\Gamma^{\prime \prime}$ is not $\mathrm{UL}^{*}$-consistent, which is impossible. Hence, we must have that $\mathcal{M}^{c}, \Gamma \Vdash \pi$.

- Rules (Epistemic Event) and (Ontic Event):

We first prove the completeness of the rule Epistemic Event . Let $\left(\Gamma, \Gamma^{\prime}, \Gamma_{f}\right) \in \mathcal{R}^{c}$, let $p \in \mathbb{P}$ and assume that $\mathcal{M}^{c}, \Gamma \Vdash p$. Assume towards a contradiction that it is not the case that $\mathcal{M}^{c}, \Gamma^{\prime \prime} \Vdash p(*)$. Then, $p \notin \Gamma^{\prime \prime}$ by the truth Lemma of [12]. However, $p \in \Gamma$ again by the truth Lemma of the companion article [12], because $\mathcal{M}^{c}, \Gamma \Vdash p$ by assumption. Moreover, $T \in \Gamma^{\prime}$, because $\vdash T$ and the Cut Lemma of the companion article [12]. Therefore, $p \otimes_{3} \top \in \Gamma^{\prime \prime}$ by definition of $\mathcal{R}^{c}$. Now, $p \vdash p$, so $p,{ }_{3} \top \vdash p$ by the rule (Epistemic Event). Hence, $p \otimes_{3} \top \vdash p$ and thus $p \in \Gamma^{\prime \prime}$ by the Cut Lemma of the companion article [12]. This contradicts $(*)$. Therefore, $\mathcal{M}^{c}, \Gamma^{\prime \prime} \Vdash p$. Reciprocally, we derive $\mathcal{M}^{c}, \Gamma \Vdash p$ from $\mathcal{M}^{c}, \Gamma^{\prime \prime} \Vdash p$ using the second inference rule of Epistemic Event).

The proof for the rule (Ontic Event) is completely similar to the rule Epistemic Event).

Rule (Epistemic Event $)$ illustrates the fact that we deal as in the standard framework of DEL with epistemic events, i.e. events which do not change atomic facts, unlike ontic events. Rule Ontic Event captures the case of ontic events, i.e. events which change the truth value of atomic facts (depending on a condition $\varphi$ ). The reading of the rule (Back-update $)$ is as follows: if in a situation satisfying $\varphi$ the occurrence of an event satisfying $\chi$ results in a final situation satisfying $\psi$, then in any situation where an agent knows that $\varphi$ holds, the occurrence of any event where he knows that $\chi$ holds will result in a final situation where he knows that $\psi$ holds. Somehow, this corresponds intuitively to a dynamic version of the rule of necessitation of epistemic logic [38]. As for rule (Forth-update), it turns out that the informal motivations for the definition of the DEL product update by Baltag \& Al. [20] are somehow formalized by this rule. Here is how 
the product update was informally motivated in this article (the notations in this quotation are replaced by our notations):

"The update product restricts the full Cartesian product $W \times W^{\mathbb{A}}$ to the smaller set $W \otimes W^{\mathbb{A}}$ in order to insure that states survive actions in the appropriate sense. [...] The components of our $\mathcal{L}^{\mathbb{A}}$-models are "simple actions", so the uncertainty regarding the action is assumed to be independent of the uncertainty regarding the current (input) state. This independence allows us to "multiply" these two uncertainties in order to compute the uncertainty regarding the output state: if whenever the input state is $w$, agent $j$ thinks the input might be some other state $v$, and if whenever the current action happening is $e$, agent $j$ thinks the current action might be some other action $f$, and if $v$ survives $f$, then whenever the output state $(w, e)$ is reached, agent $j$ thinks the alternative output state $(v, f)$ might have been reached." [20, p. 194]

Now, if one thinks of the formulas $\varphi, \chi$ and $\psi$ in the premise of rule (Forth-update as representing respectively the input state $v$, the action $f$ and the output state $(v, f)$, then the conclusion of this rule somehow formalizes these informal motivations.

Note that the rules (Forth-update) and Back-update) correspond to rules $\mathrm{R}_{5}$ and $\mathrm{R}_{4}$ of [8] respectively. Moreover, by combining the modal rules of $\mathrm{UL}^{*}$ and (Back-update), we can derive in $\mathrm{UL}^{*}+$ Back-update stronger inference rules, namely rules $\left(\mathrm{k}_{1}^{+}\right)$and $\left(\mathrm{k}_{2}^{+}\right)$below. These rules generalize the modal rule for sequent calculi of [40, p. 116].

$$
\frac{\Phi, \varphi \vdash \Psi}{\square_{j} \Phi, \diamond_{j} \varphi \vdash \diamond_{j} \Psi} \mathrm{k}_{1}^{+} \quad \frac{\Phi \vdash \Psi, \varphi}{\square_{j} \Phi \vdash \diamond_{j} \Psi, \square_{j} \varphi} \mathrm{k}_{2}^{+}
$$

where $\Phi \in \mathcal{S}\left(\mathbb{P}, \operatorname{Con}_{\varphi},\left\{{ }_{,_{0}},,_{3}\right\}\right)$ and $\Psi \in \mathcal{S}\left(\mathbb{P}, \operatorname{Con}_{\varphi},\left\{{ }_{0}\right\}\right)$, and $\square_{j} \Phi$ and $\diamond_{j} \Phi$ are defined inductively as follows:

$$
\square_{j} \Phi:=\left\{\begin{array}{ll}
\square_{j} \varphi & \text { if } \Phi=\varphi, \\
\left(\square_{j} Y,{ }_{0} \square_{j} Z\right) & \text { if } \Phi=\left(Y,,_{0} Z\right), \\
\left(\square_{j} Y,_{3} \square_{j} Z\right) & \text { if } \Phi=\left(Y,{ }_{3} Z\right),
\end{array} \diamond_{j} \Phi:= \begin{cases}\diamond_{j} \varphi & \text { if } \Phi=\varphi, \\
\left(\diamond_{j} Y,_{0} \diamond_{j} Z\right) & \text { if } \Phi=\left(Y,,_{0} Z\right) .\end{cases}\right.
$$

Note that our Epistemic Event, Ontic Event , Exclusivity, , Precondition and (Forth-update are model-complete and not frame-complete, unlike (Back-update):

Proposition 7 (Definability). The inference rule (Back-update) defines on the class of update frames the corresponding property spelled out in Figure 12

Proof. A first proof of this proposition is provided by Theorem 20 of the companion article [12], because the inference rule (Back-update) is of the form of Expression 17. Moreover, the corresponding first-order constraint established in Example 3 of the companion article [12] corresponds to the condition Back-update of Figure 12 .

Also, we provide a second direct proof of this result. We have to show that for all update frames $\mathcal{F}$, we have that $\mathcal{F}$ satisfies the condition Back-update of Figure 12 if, and only if, $\mathcal{F}$ 
validates the rule Back-update). The left to right direction is routine, so we only prove the right to left direction. First, observe that we can prove the following theorem in $\mathrm{UL}+$ (Back-update), using $\otimes_{R}^{3}$ and $\otimes_{L}^{3}$ :

$$
\square_{j} \varphi \otimes_{3} \square_{j} \alpha \vdash \square_{j}\left(\varphi \otimes_{3} \alpha\right) \quad \text { (Back-update”) }
$$

Now, we reason by contraposition. Assume that an update frame $\mathcal{F}=\left(\mathcal{P}, \mathcal{R}_{1}, \ldots, \mathcal{R}_{m}, \mathcal{R}\right)$ does not satisfy (Back-update) of Figure 12. Then, there is $\left(w, e, w_{f}\right) \in \mathcal{R}$, there is $v_{f} \in \mathcal{R}_{j}\left(w_{f}\right)$ such that for all $v \in \mathcal{R}_{j}(w)$, all $f \in \mathcal{R}_{j}(e),\left(v, f, v_{f}\right) \notin \mathcal{R}$. Then, we set an interpretation $\mathcal{I}$ on $\mathcal{F}$ such that, for a chosen $p \in \mathbb{P}$ and a chosen $\pi \in \mathcal{L}^{\mathbb{P}}, p \in \mathcal{I}(u)$ iff $u \in \mathcal{R}_{j}(w)$ and $p_{\pi} \in \mathcal{I}(u)$ iff $u \in \mathcal{R}_{j}(e)(* *)$. Then, $(\mathcal{F}, \mathcal{I}), w_{f} \Vdash \square_{j} p \otimes_{3} \square_{j} p_{\pi}$ because $\left(w, e, w_{f}\right) \in \mathcal{R}$. However, it holds that $(\mathcal{F}, \mathcal{I}), w_{f} \Vdash \diamond_{j} \neg\left(p \otimes_{3} p_{\pi}\right)$ because $v_{f} \in \mathcal{R}_{j}\left(w_{f}\right)$ and $(\mathcal{F}, \mathcal{I}), v_{f} \Vdash \neg\left(p \otimes_{3} p_{\pi}\right)$ by condition $(* *)$. Hence, $\mathcal{F}$ does not validate $\square_{j} \varphi \otimes_{3} \square_{j} \chi \vdash \square_{j}\left(\varphi \otimes_{3} \chi\right)$, that is, $\mathcal{F}$ does not validate (Back-update"). This contradicts the fact that (Back-update") is a theorem of $\mathrm{UL}+$ Back-update). Hence, we obtain our result.

\subsubsection{Characterization of the DEL Product Update}

The following theorem shows that the DEL product update is determined and characterized (modulo bisimulation) by the axioms and inference rules Epistemic Event, , Precondition, (Exclusivity), Forth-update, Back-update:

Theorem 8 (Product Update Characterization). Let $\mathcal{M}=\left(\mathcal{P}, \mathcal{R}_{1}, \ldots, \mathcal{R}_{m}, \mathcal{R}, \mathcal{I}\right)$ be a substructural model. Then,

$\mathcal{M}$ validates (Exclusivity), Precondition, Epistemic Event, (Forth-update, Back-update)

iff

$\mathcal{M}$ is isomorphic to a DEL substructural model.

Moreover, if $t$ is such an isomorphism, we have that for all $x, y, z \in \mathcal{P}$,

$$
\mathcal{R} x y z \text { iff } t(x) \otimes t(y) \leftrightarrow t(z) .
$$

Proof. The bottom to top direction is without difficulty. To prove the top to bottom direction, we use the correspondence results of Theorem 6. The isomorphism is such that if $x \in \mathcal{M}$, the pointed $\mathcal{L}^{\mathbb{P}}$-model (or $\mathcal{L}^{\mathbb{A}}$-model) $f(x)$ associated to $x$ is the $\mathcal{L}^{\mathbb{P}}$-model (or $\mathcal{L}^{\mathbb{A}}$-model) generated from the point $x$ in $\mathcal{M}$ by the accessibility relations $\left\{\mathcal{R}_{j} \mid j \in \mathbb{G}\right\}$. The preconditions for $\mathcal{L}^{\mathbb{A}}$-models are determined by the unique atomic events $p_{\pi}$ that hold at each point, because of the condition Exclusivity).

Then, we define the relation $Z \subseteq\{(\mathrm{t}(x) \otimes \mathrm{t}(y)) \times \mathrm{t}(z) \mid x, y, z \in \mathcal{M}\}$ as follows:

$$
(\mathrm{t}(x) \otimes \mathrm{t}(y)) Z \mathrm{t}(z) \quad \text { iff } \quad(x, y, z) \in \mathcal{R}
$$

One can easily show that it is a bisimulation relation. The proof for this last fact relies on the model/frame conditions (Precondition, , Epistemic Event, , Forth-update) and Back-update) of Figure 12 which correspond respectively to the (Atom), (Back) and (Forth) properties defining a bisimulation. 
As our denomination suggests, the conditions (Back-update and (Forth-update somehow encode respectively the back and forth clauses of bisimulation; the condition (Epistemic Event) somehow encodes the Atom clause of bisimulation. This result generalizes the correspondence result for public announcement logic [79] and solves an open problem raised by van Benthem [103]. Note that we also had conditions resembling the back and forth conditions of the definition of bisimulation for the specific case of public announcement [99].

\subsection{A Sequent Calculus for DEL}

Putting all our results together, we obtain a sequent calculus for DEL which is different from the calculus of [8, 9]. The advantages of having a sequent calculus for DEL are numerous (see [80, Chapter 1] for instance for more details and explanations). In particular, we do not need as in [8, 9] to resort to an external calculus to take into account the base epistemic or event logic: this base calculus is already present in the calculus at the same level as the other connectives of the language, and this base calculus can also be weakened or strengthened by appropriate structural rules.

Definition 27 (DEL sequent calculus). The DEL sequent calculus for $\mathcal{C}_{\mathrm{DEL}}^{*}$, denoted DEL ${ }^{*}$, is defined by DEL $\mathrm{UL}^{*}+\{$ Epistemic Event, Exclusivity $]$, Precondition, , Back-update, , Forth-update $\}$.

Theorem 9 (Soundness and strong completeness). The sequent calculus DEL ${ }^{*}$ is sound and strongly complete for the logic $\left(\mathcal{C}_{D E L}^{*}, E_{D E L}, \Vdash\right)$.

Proof. The soundness is routine. The completeness proof is similar to the completeness proof of Theorem 4 and ultimately relies on Theorem 8 to show that the ternary relation fulfills the condition of Definition 24

Remark 2. What we axiomatize with our proof calculi is in fact DEL without protocol, because of our very definition of DEL substructural models. It turns out that DEL with protocol has been studied in the literature [54, 105, 115, 111]. In principle, it would be possible to add specific inference/structural rules to our calculus to recover the setting of DEL with protocol, using the correspondence results of the companion article [12]. Nevertheless, an exact connection between the two approaches remains to be done.

\section{DEL Connectives are Substructural Connectives}

In this section, we will show that the DEL connectives introduced in [8, 9] correspond to the substructural connectives $\otimes_{3}, \supset_{1}, \subset_{2}, \otimes_{1}$ and $\otimes_{2}$. We will also relate the work of van Benthem on dynamic inference with the DEL-sequents of [8, 9, 15]. Finally, we will show that DEL is as expressive as epistemic logic if the number of atomic events is finite.

\subsection{Dynamic Inferences and DEL-sequents}


Dynamic Inferences In the so-called 'dynamic turn', van Benthem was interested in various dynamic styles of inference where propositions are procedures changing information states. These dynamic styles of inference differ greatly from the classical Tarskian's valid inferences because the latter are supposed to transmit and preserve truth. Among various dynamic styles of inference (such as the so-called test-test, update-update or update-test consequence [96, 97, 70]), he studied the concrete following one, which can be defined within the DEL framework:

Definition 28 (Dynamic inference, [98]). Let $\varphi_{1}, \ldots, \varphi_{n}, \varphi \in \mathcal{L}^{\mathbb{P}}$. We define the dynamic inference $\varphi_{1}, \ldots, \varphi_{n} \models \varphi$ as follows:

$$
\begin{aligned}
\varphi_{1}, \ldots, \varphi_{n} \models \varphi \quad \text { iff } \quad & \text { for all pointed } \mathcal{L}^{\mathbb{P}} \text {-model }(\mathcal{M}, w), \text { and public announcement } \\
& \mathcal{L}^{\mathbb{A}} \text {-models }\left(\mathcal{A}_{1}, e_{1}\right), \ldots,\left(\mathcal{A}_{n}, e_{n}\right) \text { of } \varphi_{1}, \ldots, \varphi_{n} \text { respectively, } \\
& (\mathcal{M}, w) \otimes\left(\mathcal{A}_{1}, e_{1}\right) \otimes \ldots \otimes\left(\mathcal{A}_{n}, e_{n}\right) \models \varphi
\end{aligned}
$$

where the public announcement $\mathcal{L}^{\mathbb{A}}$-model of $\psi$ is the pointed $\mathcal{L}^{\mathbb{A}}$-model of Figure 3 , with $r_{A}$ replaced by $\psi$.

Van Benthem noticed that various dynamic styles of inference obey structural rules of inference which are non-classical. For example, all the structural rules of classical logic of Figure 5 fail for dynamic inference, but the structural rules below characterize completely the dynamic inference [98] (below, $\vec{\psi}$ stands for $\varphi_{1}, \ldots, \varphi_{n}$ and $\vec{\psi}$ stands for $\psi_{1}, \ldots, \psi_{n}$, where $\left.\varphi_{1}, \ldots, \varphi_{n}, \psi_{1}, \ldots, \psi_{n} \in \mathcal{L}^{\mathbb{P}}\right)$ :

$$
\begin{aligned}
& \text { if } \vec{\varphi} \models \varphi \text { then } \psi, \vec{\varphi} \models \varphi \\
& \text { if } \vec{\varphi} \models \varphi \text { and } \vec{\varphi}, \varphi, \vec{\psi} \models \psi \text { then } \vec{\varphi}, \vec{\psi} \models \psi \\
& \text { if } \vec{\varphi} \models \varphi \text { and } \vec{\varphi}, \vec{\psi} \models \psi \text { then } \vec{\varphi}, \varphi, \vec{\psi} \models \psi
\end{aligned}
$$

(Left-Monotonicity)

(Cautious Monotonicity)

In the next section, we will explain why some substructural phenomena arise in these dynamic inferences. But before that, we need to show that dynamic inferences are in fact instances of a more general kind of inference called DEL-sequents.

DEL-sequents They were introduced in [8]. They are a particular sort of dynamic inference and are defined as follows:

Definition 29 (DEL-sequent, [8]). Let $\varphi, \psi \in \mathcal{L}^{\mathbb{P}}$ and $\chi \in \mathcal{L}^{\mathbb{A}}$. We define the logical consequence relation $\varphi, \chi \models \psi$ as follows:

$$
\varphi, \chi \models \psi \quad \text { iff } \quad \text { for all pointed } \mathcal{L}^{\mathbb{P}} \text {-model }(\mathcal{M}, w) \text {, all pointed } \mathcal{L}^{\mathbb{A}} \text {-model }(\mathcal{A}, e) \text { such that }
$$

$$
\mathcal{M}, w \models I^{\mathbb{A}}(e) \wedge \varphi \text { and } \mathcal{A}, e \models \chi \text {, it holds that }(\mathcal{M}, w) \otimes(\mathcal{A}, e) \models \psi \text {. }
$$

In [15], DEL-sequents are generalized to take into account sequences of events and not only 'one-shot' occurrence of events. Several generalized DEL-sequents are introduced in [15] but they are all reducible to the following one: 
Definition 30 (Generalized DEL-sequent, [15]). Let $\varphi_{0}, \ldots, \varphi_{n} \in \mathcal{L}^{\mathbb{P}}$, let $\chi_{1}, \ldots, \chi_{n} \in \mathcal{L}^{\mathbb{A}}$ and let $\psi \in \mathcal{L}^{\mathbb{P}}$. Then,

$$
\varphi_{0}, \chi_{1}, \varphi_{1}, \ldots, \chi_{n}, \varphi_{n} \models \psi
$$

iff

if for all pointed $\mathcal{L}^{\mathbb{P}}$-model $(\mathcal{M}, w)$, and $\mathcal{L}^{\mathbb{A}}$-models $\left(\mathcal{A}_{1}, e_{1}\right), \ldots,\left(\mathcal{A}_{n}, e_{n}\right)$ such that for all $i \in\{1, \ldots, n\}, \mathcal{A}_{i}, e_{i}=\chi_{i},(\mathcal{M}, w) \otimes\left(\mathcal{A}_{1}, e_{1}\right) \otimes \ldots \otimes\left(\mathcal{A}_{i}, e_{i}\right)$ is defined and makes $\varphi_{i}$ true, then it holds that $(\mathcal{M}, w) \otimes\left(\mathcal{A}_{1}, e_{1}\right) \otimes \ldots \otimes\left(\mathcal{A}_{n}, e_{n}\right) \models \psi$.

As one can easily notice, dynamic inferences can be translated into DEL-sequents if we resort to the common knowledge/belief operator $\square_{\mathbb{G}}^{*} \varphi$ (see for example Fagin \& Al. [38] for a definition and a detailed study of this operator):

Proposition 10. Let $\varphi_{0}, \varphi_{1}, \ldots, \varphi_{n}, \varphi \in \mathcal{L}^{\mathbb{P}}$. Then, the following holds:

$$
\varphi_{1}, \ldots, \varphi_{n} \models \varphi \quad \text { iff } \quad \top, p_{\varphi_{1}} \wedge \square_{\mathbb{G}}^{*} p_{\varphi_{1}}, \ldots, \top, p_{\varphi_{n}} \wedge \square_{\mathbb{G}}^{*} p_{\varphi_{n}}, \top \models \varphi \wedge \square_{\mathbb{G}}^{*} \varphi
$$

Thus, DEL-sequents are more expressive than dynamic inferences, and also more abstract because they 'operate' at a deeper level, a semantical one. It is this more general and abstract approach towards dynamic styles of inference that will allow us to relate more precisely and closely DEL with substructural logics, and explain to a certain extent why the substructural phenomena occurring in dynamic inferences and observed by van Benthem arise.

\subsection{DEL-sequents for Progression, Regression and Epistemic Planning}

Recently again, van Benthem [102] expressed some worries about interpreting the Lambek Calculus (the paradigmatic substructural logic) as a base logic of information flow while trying to connect the connectives $\otimes, \supset$ and $\subset$ of substructural logics to some sort of DEL connectives. Indeed, the DEL connectives usually rely on the regular algebra of sequential composition, choice and iteration which are of a quite different nature. Recently, some DEL connectives called progression, regression and epistemic planning were introduced [8, 9], the connective of regression being a natural generalization of the standard and original action modality $[\mathcal{A}, e] \varphi$ of DEL [21]. It turns out that these connectives can all be identified with connectives of our substructural language $\mathcal{L}_{\mathrm{DEL}}$. After briefly recalling their definitions and the motivations for introducing them, we give our correspondence results between the two kinds of connectives. The results of [8, 9] will allow us to obtain an interesting expressivity result for DEL (Corollary 2).

As spelled out in Section 2, the core idea of DEL is to split the task of representing the agents' beliefs into three parts. Consequently, within the logical framework of DEL, one can express uniformly epistemic statements about:

(i) what is true about an initial situation,

(ii) what is true about an event occurring in this situation,

(iii) what is true about the resulting situation after the event has occurred. 
From a logical point of view, this trichotomy gives rise to the following three questions (which were already raised by Kooi [55]). In these questions, $\varphi, \chi$ and $\psi$ are three epistemic formulas describing respectively (i), (ii) and (iii).

\section{- Question 1: Progression}

1. Given (i) and (ii), what can we infer about (iii): $\varphi, \chi \models \psi$ ?

2. How can we build a single formula $\varphi \otimes \chi$ which captures all the information which can be inferred about (iii) from $\varphi$ and $\chi$ ?

\section{- Question 2: Epistemic Planning}

1. Given (i) and (iii), what can we infer about (ii): $\varphi, \psi \models \chi$ ?

2. How can we build a single formula $\varphi \theta_{P} \psi$ which captures all the information which can be inferred about (ii) from $\varphi$ and $\psi$ ?

\section{- Question 3: Regression}

1. Given (ii) and (iii), what can we infer about (i): $\chi, \psi \models \varphi$ ?

2. How can we build a single formula $\chi \oslash \psi$ which captures all the information which can be inferred about (i) from $\chi$ and $\psi$ ?

Note that these three questions are of the form $(a)$ (we defined these types of questions informally on page 15 ). Providing formal tools that answer these questions leads to applications in artificial intelligence and theoretical computer science, and as it turns out, some of these questions have already been addressed in DEL and other logical formalisms (see [8] for more details).

- Question 1: Progression. Answering the first question leads to the development of tools that can be used by (artificial) agents to compute autonomously their representation of situations as events occur or to reason about the effects of these events. This question has been addressed in the situation calculus, where it is related to the notion of progression [84]. In the logics of programs, our DEL-sequent $\varphi, \chi \models \psi$ correspond to the partial correctness specifications $\{\varphi\} \pi\{\psi\}$ of Hoare's logic [53] which read as "after every successful execution of program $\pi$ starting from a state where precondition $\varphi$ holds, postcondition $\psi$ holds in the final state". Likewise, our formula $\varphi \otimes \chi$ corresponds to the strongest postcondition of Propositional Dynamic Logic [82]. That the product update of DEL is in fact the same as the strongest post-condition has been elaborated on and proved in an algebraic setting by Baltag \& Al. [18]. A sequent calculus is also provided in this algebraic setting.

- Question 2: Epistemic planning. Answering the second question also leads to applications in artificial intelligence in the area of epistemic planning: (artificial) agents often need to determine autonomously which actions they need to perform in order to achieve a given epistemic goal. This second question is also related to the notion of explanation and has been dealt with in the event calculus [92] for instance, where it is shown that planning problems can be handled via abduction (using logic programming). In computer science, 
this second question is also related to the synthesis problem raised by Church [35] in its full generality. He asked whether, given a desired relation between a set of inputs and a set of outputs, we can construct a function that produces the desired outputs from arbitrary inputs. This problem has been declined as the problem of program synthesis: given a specification, can we construct a program that is guaranteed to satisfy this specification? It was extensively studied in the 1980s and 1990s for temporal logic specifications. The synthesis problem is more challenging when the input is incomplete [57]. Open (reactive) environments can be a reason of incompleteness of the input, and epistemic logic is a natural formalism to resort to model such situations, as argued by Halpern and Moses [51]. For single-agent temporal epistemic logic, this synthesis problem has been solved by van der Meyden and Vardi [108].

- Question 3: Regression. Answering the third question is related to the notion of regression introduced in the situation calculus [84]. This technique is used to determine whether a statement holds after a sequence of events (called the projection problem) by reducing (regressing) this statement about the resulting situation to a statement about the initial situation. In DEL, regression corresponds to the classical reduction method used to prove completeness of an axiomatization: a formula with dynamic operator(s) is 'reduced' equivalently to a formula without dynamic operator by pushing the dynamic operator through the logical connectives, performing some kind of regression of the initial formula with dynamic operator. In [9], our inductive definition of the regression of $\psi$ by $\chi$, i.e. $\chi \oslash \psi$, is based on the reduction axioms of DEL [20]. Note that in Propositional Dynamic Logic, $\neg(\varphi \oslash \neg \psi)$ also corresponds to the weakest precondition.

Now, we provide the formal definitions of these connectives of progression, regression and epistemic planning.

Progression The connective of progression is denoted $\otimes$ in [8]. In [9, Definition 41], a constructive definition of this connective is provided using characteristic formulas (called "Kit Fine" formulas). Here, we provide an alternative and non-constructive definition of the progression of $\varphi$ by $\chi$, denoted $\varphi \otimes \chi$ :

Theorem 11. Let $\left(\mathcal{M}_{f}, w_{f}\right)$ be a pointed $\mathcal{L}^{\mathbb{P}}$-model and let $\varphi \in \mathcal{L}^{\mathbb{P}}$ and $\chi \in \mathcal{L}^{\mathbb{A}}$. Then,

$$
\mathcal{M}_{f}, w_{f} \models \varphi \otimes \chi \quad \text { iff } \quad \begin{aligned}
& \text { there is a pointed } \mathcal{L}^{\mathbb{P}} \text {-model }(\mathcal{M}, w) \text { and a pointed } \\
& \mathcal{L}^{\mathbb{A}} \text {-model }(\mathcal{A}, e) \text { such that }(\mathcal{M}, w) \otimes(\mathcal{A}, e) \leftrightarrows\left(\mathcal{M}_{f}, w_{f}\right), \\
& \\
& \mathcal{M}, w \models \varphi \text { and } \mathcal{A}, e \models \chi
\end{aligned}
$$

Proof. It follows from Lemmata 43 and 44 of [8].

Epistemic Planning The connective of epistemic planning is denoted $Q_{P}$ in [9]. It is defined relatively to a finite set $P$ of formulas/preconditions/atomic events. In [9, Definition 14-15], a constructive definition of this connective is provided using characteristic formulas (called "Kit Fine" formulas). As it turns out, an alternative and non-constructive definition of the epistemic planning from $\varphi$ to $\psi$, denoted $\varphi \theta_{P} \psi$, exists as well: 
Theorem 12 ([9]). Let $\varphi, \psi \in \mathcal{L}^{\mathbb{P}}$ and let $P$ be a finite subset of $\mathcal{L}^{\mathbb{P}}$. Then, for all P-complete $\mathcal{L}^{\mathbb{A}}$-model $(\mathcal{A}, e)$, it holds that

$$
\begin{array}{ll}
\mathcal{A}, e \models \varphi \otimes_{P} \psi \quad \text { iff } \quad \text { there is }(\mathcal{M}, w) \text { such that } \mathcal{M}, w \models \varphi, \\
\mathcal{M}, w \models I^{\mathbb{A}}(e) \text { and }(\mathcal{M}, w) \otimes(\mathcal{A}, e) \models \psi
\end{array}
$$

The dual of the connective $\varphi \theta_{P} \psi$ is defined by:

$$
\varphi[\Theta]_{P} \psi:=\neg\left(\varphi \Theta_{P} \neg \psi\right)
$$

Theorem 12 entails that $\varphi[\theta]_{P} \psi$ can be alternatively defined as follows: for all $P$-complete $\mathcal{L}^{\mathbb{A}}$-model $(\mathcal{A}, e)$, it holds that

$$
\begin{gathered}
\mathcal{A}, e \models \varphi[\theta]_{P} \psi \text { iff } \text { for all }(\mathcal{M}, w) \text { such that } \mathcal{M}, w \models \varphi \text {, if } \\
\qquad \mathcal{M}, w \models I^{\mathbb{A}}(e) \text { then }(\mathcal{M}, w) \otimes(\mathcal{A}, e) \models \psi
\end{gathered}
$$

Example 7. In the situation depicted in the $\mathcal{L}^{\mathbb{P}}$-model of Figure 1 , agent $\mathrm{B}$ does not know that agent $\mathrm{A}$ has the red card and does not know that agent $\mathrm{C}$ has the blue card: $\mathcal{M}, w \models\left(\diamond_{B} r_{A} \wedge\right.$ $\left.\diamond_{B} \neg r_{A}\right) \wedge\left(\diamond_{B} b_{C} \wedge \diamond_{B} \neg b_{C}\right)$. Our problem is therefore the following:

What sufficient and necessary property (i.e. 'minimal' property) an event should fulfill so that its occurence in the initial situation $(\mathcal{M}, w)$ results in a situation where agent $\mathrm{B}$ knows the true state of the world, i.e. agent $\mathrm{B}$ knows that agent $\mathrm{A}$ has the red card and that agent $\mathrm{C}$ has the blue card?

The answer to this question obviously depends on the kind of atomic events we consider. In this example, the events $P=\left\{p_{b_{C}}, p_{r_{A}}, p_{w_{B}}\right\}$ under consideration are the following. First, agent $\mathrm{C}$ shows her blue card $\left(p_{b_{C}}\right)$, second, agent $\mathrm{A}$ shows her red card $\left(p_{r_{A}}\right)$, and third, agent $\mathrm{B}$ herself shows her white card $\left(p_{w_{B}}\right)$. Answering this question amounts to compute the formula $(M, w) \otimes_{P} \square_{B}\left(r_{A} \wedge b_{C} \wedge w_{B}\right)$. Applying the algorithm of [9, Definition 15], we obtain that

$$
(\mathcal{M}, w) Q_{P} \square_{B}\left(r_{A} \wedge b_{C} \wedge w_{B}\right) \leftrightarrow \square_{B}\left(p_{b_{C}} \vee p_{r_{A}}\right) \text { is valid. }
$$

In other words, this result states that agent $B$ should believe either that agent $A$ shows her red card or that agent $C$ shows her blue card in order to know the true state of the world. Indeed, since there are only three different cards which are known by the agents and agent B already knows her card, if she learns the card of (at least) one of the other agents, she will also be able to infer the card of the third agent.

Regression The connective of regression is denoted $\oslash$ in [8]. In [9, Def. 41], a constructive definition of this connective is provided using characteristic formulas (called "Kit Fine" formulas) by adapting and translating the reduction axioms of [21]. As it turns out, an alternative and non-constructive definition of the regression of $\psi$ by $\chi$, denoted $\chi \oslash \psi$, exists as well:

Theorem 13 ([9]). Let $\chi \in \mathcal{L}^{\mathbb{A}}$ and $\psi \in \mathcal{L}^{\mathbb{P}}$. Then, for all $\mathcal{L}^{\mathbb{P}}$-model $(\mathcal{M}, w)$, it holds that

$$
\begin{gathered}
\mathcal{M}, w \models \chi \oslash \psi \quad \text { iff } \quad \begin{array}{l}
\text { there is }(\mathcal{A}, e) \text { such that } \mathcal{A}, e \models \chi, \\
\mathcal{M}, w \models I^{\mathbb{A}}(e) \text { and }(\mathcal{M}, w) \otimes(\mathcal{A}, e) \models \psi
\end{array}
\end{gathered}
$$


Note that we could define a dual connective of $\chi \oslash \psi$ as follows:

$$
\chi[\oslash] \psi=\neg(\chi \oslash \neg \psi)
$$

Then, the counterpart of Theorem 13 for this dual connective is as follows:

$$
\begin{aligned}
\mathcal{M}, w \models \chi[\oslash] \psi \quad \text { iff } \quad \text { for all }(\mathcal{A}, e) \text { such that } \mathcal{A}, e \models \chi & \text { if } \mathcal{M}, w \models I^{\mathbb{A}}(e) \text { then }(\mathcal{M}, w) \otimes(\mathcal{A}, e) \models \psi
\end{aligned}
$$

As shown in [9, Sec. 6], the connective $\chi[\oslash] \psi$ is a generalization of the original and more standard DEL connective $[\mathcal{A}, e] \varphi$ almost exclusively used in the DEL literature [21].

Correspondence between DEL and Substructural Connectives As one can easily notice, there is a strong similarity between the operations of progression, epistemic planning and regression and the operations of substructural logics, more precisely of the Lambek Calculus. In fact, there exists a rigorous mapping between them, as the following theorem shows:

Theorem 14. Let $\mathcal{M}_{D E L}$ be the full DEL substructural model. Let $P$ be a finite subset of $\mathcal{L}^{\mathbb{P}}$, let $x=(\mathcal{M}, w) \in \mathcal{E}^{\mathbb{P}}$ and let $y=(\mathcal{A}, e) \in \mathcal{E}_{P}^{\mathbb{A}}$ be a P-complete pointed event model. Let $\varphi, \psi \in \mathcal{L}^{\mathbb{P}}$ and let $\chi \in \mathcal{L}^{\mathbb{A}}$. Then,

$$
\begin{array}{lll}
\mathcal{M}_{D E L}, x \Vdash \chi \otimes_{1} \varphi & \text { iff } & \mathcal{M}, w \models \chi \oslash \varphi \\
\mathcal{M}_{D E L}, y \| \psi \otimes_{2} \varphi & \text { iff } & \mathcal{A}, e \models \varphi \otimes_{P} \psi \\
\mathcal{M}_{D E L}, x \Vdash \varphi \otimes_{3} \chi & \text { iff } & \mathcal{M}, w \models \varphi \otimes \chi
\end{array}
$$

Moreover, for all $\chi, \chi_{1}, \ldots, \chi_{n} \in \mathcal{L}^{\mathbb{A}}$, for all $\varphi, \psi, \varphi_{0}, \varphi_{1}, \ldots, \varphi_{n} \in \mathcal{L}^{\mathbb{P}}$, we have:

$$
\begin{array}{lll}
\varphi,,_{3} \chi \Vdash \psi & \text { iff } & \varphi, \chi \models \psi \\
\left(\left(\left(\varphi_{0},{ }_{3} \chi_{1}\right), \varphi_{1}\right),{ }_{3} \ldots,{ }_{3} \chi_{n}\right), \varphi_{n} \Vdash \psi & \text { iff } & \varphi_{0}, \chi_{1}, \varphi_{1}, \ldots, \chi_{n}, \varphi_{n} \models \psi
\end{array}
$$

Proof. It follows straightforwardly from Theorems 11, 12, 13 and the truth conditions of the connectives $\otimes_{3}, \supset_{1}, \subset_{2}$ and ${ }_{, 3}$.

Theorem 14 explains why some substructural phenomena arise in the dynamic inferences of Section 7.1. As observed by van Benthem, "it seemed that structural rules address mere symptoms of some underlying phenomenon" [103, p. 297]. We claim that these "symptoms" are caused at a deeper semantic level by the fact that an update, and in that case the DEL product update, can be represented by the ternary relation of substructural logics.

The key Theorem 42 of [8] relates DEL-sequents and the connective of progression: for all $\varphi, \psi \in \mathcal{L}^{\mathbb{P}}$ and $\chi \in \mathcal{L}^{\mathbb{A}}$, it holds that

$$
\varphi, \chi \models \psi \text { iff } \varphi \otimes \chi \models \psi .
$$

As it turns out, this theorem is also valid in any substructural logics. More generally, all the theorems of the non-associative Lambek calculus hold in our DEL setting if we use the translation given in Figure 13. In particular, we have the following results. 


\begin{tabular}{|c|c|c|}
\hline $\begin{array}{c}\text { Substructural } \\
\text { connectives }\end{array}$ & $\begin{array}{c}\text { Update Logic } \\
\text { connectives }\end{array}$ & $\begin{array}{c}\text { DEL } \\
\text { connectives }\end{array}$ \\
\hline$\otimes$ & $\otimes_{3}$ & $\otimes$ \\
$\supset$ & $\supset_{1}$ & {$[\oslash]$} \\
$\subset$ & $C_{2}$ & {$[\oslash]$} \\
& $\otimes_{1}$ & $\oslash$ \\
& $\otimes_{2}$ & $\oslash$ \\
\hline
\end{tabular}

Figure 13: Correspondence between DEL, Update Logic and Substructural Connectives

Corollary 1. Let $P$ be a finite subset of $\mathcal{L}^{\mathbb{P}}$. For all $\varphi, \psi \in \mathcal{L}^{\mathbb{P}}$ and $\chi \in \mathcal{L}^{\mathbb{A}}$, it holds that

$$
\begin{aligned}
& \varphi,{ }_{3} \chi \Vdash \psi \quad \text { iff } \varphi \Vdash \chi[\oslash] \psi \\
& \varphi \Vdash \chi[\oslash] \psi \quad \text { iff } \varphi \otimes \chi \Vdash \psi \\
& \varphi \otimes \chi \| \psi \quad \text { iff } \chi \|^{\mathcal{E}_{P}^{\mathrm{A}}} \varphi[\theta]_{P} \psi \\
& \varphi \Vdash \chi[\oslash] \psi \quad \text { iff } \chi \|^{\mathcal{E}_{P}^{\mathrm{A}}} \varphi[\theta]_{P} \psi
\end{aligned}
$$

The corollary below essentially tells us that if we assume that the set of atomic events is finite, then DEL is as expressive as epistemic logic. In fact, this result is in line with the expressiveness result of [21] for the original DEL framework.

Corollary 2 (Expressiveness of DEL). If $\mathbb{A}$ is finite, then $\left(\mathcal{L}_{D E L}^{\mathbb{P}},\left\{\mathcal{M}_{D E L}\right\}, \Vdash\right) \equiv\left(\mathcal{L}^{\mathbb{P}}, \mathcal{E}^{\mathbb{P}}, \models\right)$ and $\left(\mathcal{L}_{D E L}^{\mathbb{A}},\left\{\mathcal{M}_{D E L}\right\}, \Vdash\right) \equiv\left(\mathcal{L}^{\mathbb{A}}, \mathcal{E}^{\mathbb{A}}, \models\right)$. Thus, the logic DEL and epistemic logic have the same expressive power.

Proof. We only prove the corollary for $\mathcal{L}_{\mathrm{DEL}}^{\mathbb{P}}$, the proof for $\mathcal{L}_{\mathrm{DEL}}^{\mathbb{A}}$ being similar. First, note that since $\mathbb{A}$ is finite, any pointed event model of $\mathcal{M}_{\mathrm{DEL}}$ is in fact an $P$-complete pointed event model, where $P:=\left\{\pi \mid p_{\pi} \in \mathbb{A}\right\}$. Morever, Definition 41 (with Theorem 42) of [8], Definition 15 (with Theorem 7) and Definition 20 (with Theorem 13) of [9] show that the formulas $\chi \oslash \varphi$, $\varphi \otimes \chi$ of Theorem 14 are equivalent to formulas of the epistemic language $\mathcal{L}^{\mathbb{P}}$ and that the formula $\varphi \theta_{P} \psi$ is equivalent to a formula of the event language $\mathcal{L}^{\mathbb{A}}$. More generally, given a formula $\varphi \in \mathcal{L}_{\mathrm{DEL}}^{\mathbb{P}}$, this formula $\varphi$ can be translated equivalently into a formula containing only the connectives $\otimes_{1}, \otimes_{2}, \otimes_{3}, \square_{j}, \wedge$ and $\neg$ using the translation $\tau$ defined inductively as follows: for all $f \in\left\{\square_{j}, \neg\right\}$ and all $f^{\prime} \in\{\wedge, \vee\}$,

$$
\begin{aligned}
\tau(p) & :=p & \tau\left(\varphi \supset_{\mathrm{j}} \psi\right) & :=\neg\left(\tau(\varphi) \otimes_{\mathrm{i}} \neg \tau(\psi)\right) \\
\tau(f \varphi) & :=f \tau(\varphi) & \tau\left(\varphi \subset_{\mathrm{i}} \psi\right) & :=\neg\left(\neg \tau(\varphi) \otimes_{\mathrm{i}} \tau(\psi)\right) \\
\tau\left(\varphi f^{\prime} \psi\right) & :=\tau(\varphi) f^{\prime} \tau(\psi) & &
\end{aligned}
$$

From this prior translation $\tau$, we can show by induction on the number of substructural connectives $\otimes_{1}, \otimes_{2}, \otimes_{3}$ that our result holds. The case $n=0$ holds trivially. For the case $n+1$, it suffices to identify within the formula $\varphi$ a subformula of the form $\chi \otimes_{1} \psi, \varphi \otimes_{2} \psi$ or $\varphi \otimes_{3} \chi$ (that 
is, respectively of the form $\chi \oslash \psi, \varphi \otimes_{P} \psi$ or $\varphi \otimes \chi$ via Theorem 14). Then, we replace this subformula $\psi$ in $\varphi$ by its appropriate translation into $\mathcal{L}^{\mathbb{P}}$ or $\mathcal{L}^{\mathbb{A}}$ as they are defined in Definition 41 of [8] or Definition 15 and Definition 20 of [9]. This yields a formula $\varphi^{\prime} \in \mathcal{L}_{\mathrm{DEL}}^{\mathbb{P}}$ equivalent to $\varphi$ and which contains $n$ substructural connectives. We can then apply the induction hypothesis to $\varphi^{\prime}$, yielding a formula $\varphi^{\prime \prime} \in \mathcal{L}^{\mathbb{P}}$ equivalent to $\varphi^{\prime}$ and therefore also equivalent to $\varphi$.

\section{Conclusion}

In this article we have shown that DEL can be embedded within the framework of substructural logics in an intuitively meaningful way, in the sense that in this embedding the intuitions underlying the DEL framework provide a reasonable and meaningful interpretation of the ternary semantics of substructural logics. In addition to other non-classical logics such as linear logic, relevance logic, arrow logic, etc. this new embedding illustrates the richness and expressiveness of the substructural framework: it is defined in such a way that it can even capture in a meaningful way logics which are sometimes considered as isolated or 'exotic', like DEL. Therefore, our results are evidence in support of the claim that the relational semantics of the substructural framework can be considered as a unifying semantical framework for non-classical logics.

We elicited a number of axioms and inference rules that define specific properties of the DEL product update. This led us to define a sequent calculus for DEL which is based on the update logic introduced in the companion article [12]. In parallel, we also provided a sequent calculus for update logic and we observed that this sequent calculus is in fact a generalization of the non-associative Lambek calculus. Fritella Et Al. [42, 43] provide a display calculus for DEL. Their version of DEL is based on the original logic of Baltag \& Al. [21, 20], where modalities are associated to pointed $\mathcal{L}^{\mathbb{A}}$-models. Their display calculus is therefore very different. It is also multi-typed, where types can be agents, (functional) actions and formulas.

While the DEL product update was the focus of our attention in this article, it is nevertheless a particular kind of update and the ternary relation of substructural logics could actually be a representation of any sort of update or any sort of logical dynamics within and beyond the DEL paradigm. Within the DEL paradigm, Liu [61] mentions some of the alternatives to the DEL product update and Eijck \& Al. [114] recently introduced a new sort of product update (subsequently studied by Aceto \& Al. [1]). Numerous product update rules for belief revision have also been proposed in the DEL paradigm with more refined representations of uncertainty (by means of plausibility or probability measures for instance) [5, 109, 6, 23, 24, 99, 61, 106]. Outside the DEL paradigm, other kinds of model changing operations have recently been investigated in various kinds of dynamic logics [56, 13, 50, 3, 4, 39, 17, 16]. An interesting line of research would be to investigate whether and how these logics can be embedded into our update logic and considered as specific instances of it. In particular, the proof techniques developed in this article would have to be adapted to these other approaches. It may turn out that we find among the structural rules elicited in the substructural logics literature (see the companion article [12]) some rules which capture the types of updates defined implicitly in these dynamic logics.

More generally, the ternary relation could also represent the various revision, update and even causal connectives which have been studied in the logics of "commonsense reasoning" in artificial intelligence and philosophical logic, such as conditional logic [74], default and non- 
monotonic logics [62, 44], belief revision theory [45], etc. Formalizing commonsense reasoning within non-classical logics is not a new line of research (see for instance [48, 76, 71, 72, 2, 75]). The originality of our approach is based on our proposal to found it on the general framework of substructural logics and more particularly on its ternary relational semantics. Some connections between causal conditionals [49] and ternary semantics have in fact already started to emerge [33]. Connections with belief revision and conditional logic can also be found in [11].

Finally, one should note that only a few of the connectives introduced were needed to capture the logical dynamics present in standard DEL (mainly $\otimes_{3}, \supset_{1}, \subset_{2}$ ). However, from our dynamic reading of the ternary relation, it is possible to provide for the other connectives of $\left\{\otimes_{\mathrm{i}}, \supset_{\mathrm{i}}, \subset_{\mathrm{i}} \mid i \in\{1,2,3\}\right\}$ an intuitive and quite meaningful reading as well. This opens our investigation to a vast new realm of logical dynamics and our approach may provide new means to formalize and study more difficult and complex forms of commonsense reasoning, such as abductive or retractive reasoning.

Acknowledgements I thank Olivier Roy and Ole Hjortland for inviting me to the Munich Meeting on Sub-structural Epistemic Logic (January 2013), which was very inspiring. I also thank Marta Bilkova for subsequent discussions. I thank Johan van Benthem and Igor Sedlar for comments on an earlier version of this article. I thank an anonymous referee for helpful and extensive comments. I thank Sean Sedwards for checking the English of this article. Finally, I thank the editors of the special issue and Jane Spurr for their patience.

\section{References}

[1] Luca Aceto, Anna Ingólfsdóttir, Cristian Prisacariu, and Joshua Sack. Compositional reasoning for multi-modal logics. In Sergei N. Artëmov and Anil Nerode, editors, LFCS, volume 7734 of Lecture Notes in Computer Science, pages 1-15. Springer, 2013.

[2] Régis Alenda, Nicola Olivetti, and Gian Luca Pozzato. Nested sequent calculi for conditional logics. In del Cerro et al. [36], pages 14-27.

[3] Carlos Areces, Raul Fervari, and Guillaume Hoffmann. Swap logic. Logic Journal of IGPL, 22(2):309-332, 2014.

[4] Carlos Areces, Raul Fervari, and Guillaume Hoffmann. Relation-changing modal operators. Logic Journal of the IGPL, 23(4):601-627, 2015.

[5] Guillaume Aucher. A combined system for update logic and belief revision. In Mike Barley and Nikola K. Kasabov, editors, PRIMA, volume 3371 of Lecture Notes in Computer Science, pages 1-17. Springer, 2004.

[6] Guillaume Aucher. Interpreting an action from what we perceive and what we expect. Journal of Applied Non-Classical Logics, 17(1):9-38, 2007.

[7] Guillaume Aucher. Perspectives on belief and change. PhD thesis, University of Otago University of Toulouse, 2008. 
[8] Guillaume Aucher. DEL-sequents for progression. Journal of Applied Non-Classical Logics, 21(3-4):289-321, 2011.

[9] Guillaume Aucher. DEL-sequents for regression and epistemic planning. Journal of Applied Non-Classical Logics, 22(4):337-367, 2012.

[10] Guillaume Aucher. DEL as a substructural logic. In Alexandru Baltag and Sonja Smets, editors, Outstanding Contributions: Johan F. A. K. van Benthem on Logical and Informational Dynamics, Trends in Logic. Springer, 2014.

[11] Guillaume Aucher. When conditional logic and belief revision meet substructural logics. In Richard Booth, Giovanni Casini, Szymon Klarman, Gilles Richard, and Ivan José Varzinczak, editors, Proceedings of the International Workshop on Defeasible and Ampliative Reasoning, DARe 2015, co-located with the 24th International Joint Conference on Artificial Intelligence (IJCAI 2015), Buenos Aires, Argentina, July 27, 2015., volume 1423 of CEUR Workshop Proceedings. CEUR-WS.org, 2015.

[12] Guillaume Aucher. Displaying updates in logic. Journal of Logic and Computation, Special Issue on Substructural Logics and Information Dynamics(doi:10.1093/logcom/exw001), 2016.

[13] Guillaume Aucher, Philippe Balbiani, Luis Fariñas Del Cerro, and Andreas Herzig. Global and local graph modifiers. In Methods for Modalities 5 (M4M-5), Cachan, France, 2007. ENTCS, Elsevier.

[14] Guillaume Aucher and Andreas Herzig. Exploring the power of converse events. Dynamic Formal Epistemology, pages 51-74, 2011.

[15] Guillaume Aucher, Bastien Maubert, and François Schwarzentruber. Generalized DELsequents. In del Cerro et al. [36], pages 54-66.

[16] Philippe Balbiani, Rachid Echahed, and Andreas Herzig. A dynamic logic for termgraph rewriting. In Hartmut Ehrig, Arend Rensink, Grzegorz Rozenberg, and Andy Schürr, editors, ICGT, volume 6372 of Lecture Notes in Computer Science, pages 59-74. Springer, 2010.

[17] Philippe Balbiani, Andreas Herzig, and Nicolas Troquard. Dynamic logic of propositional assignments: A well-behaved variant of PDL. In LICS, pages 143-152. IEEE Computer Society, 2013.

[18] Alexandru Baltag, Bob Coecke, and Mehrnoosh Sadrzadeh. Algebra and sequent calculus for epistemic actions. Electronic Notes in Theoretical Computer Science, 126:27-52, 2005.

[19] Alexandru Baltag, Bob Coecke, and Mehrnoosh Sadrzadeh. Epistemic actions as resources. Journal of Logic and Computation, 17(3):555-585, 2007. 
[20] Alexandru Baltag and Lawrence S. Moss. Logic for epistemic programs. Synthese, 139(2):165-224, 2004.

[21] Alexandru Baltag, Lawrence S. Moss, and Slawomir Solecki. The logic of public announcements and common knowledge and private suspicions. In Itzhak Gilboa, editor, TARK, pages 43-56. Morgan Kaufmann, 1998.

[22] Alexandru Baltag, Lawrence S. Moss, and Slawomir Solecki. The logic of public announcements, common knowledge and private suspicions. Technical report, Indiana University, 1999.

[23] Alexandru Baltag and Sonja Smets. Conditional doxastic models: A qualitative approach to dynamic belief revision. Electronic Notes in Theoretical Computer Science, 165:5-21, 2006.

[24] Alexandru Baltag and Sonja Smets. Probabilistic dynamic belief revision. Synthese, 165(2):179-202, 2008.

[25] Alexandru Baltag and Sonja Smets. Texts in Logic and Games, volume 4, chapter The Logic of Conditional Doxastic Actions, pages 9-31. Amsterdam University Press, 2008.

[26] Alexandru Baltag and Sonja Smets. Texts in Logic and Games, volume 3, chapter A Qualitative Theory of Dynamic Interactive Belief Revision, pages 9-58. Amsterdam University Press, 2008.

[27] John Barwise and John Perry. Situations and Attitudes. Cambridge, Massachusetts. MIT Press, 1983.

[28] Jon Barwise. Constraints, channels, and the flow of information. Situation theory and its applications, 3:3-27, 1993.

[29] Jon Barwise, Dov Gabbay, and Chrysafis Hartonas. Information flow and the lambek calculus. In Logic, Language and Computation: The 1994 Moraga Proceedings, ed. by Jerry Seligman and Dag Westerstahl. Forthcoming from CSLI, pages 49-64, 1994.

[30] Jon Barwise, Dov Gabbay, and Chrysafis Hartonas. On the logic of information flow. Logic Journal of IGPL, 3(1):7-49, 1995.

[31] Jc Beall, Ross Brady, J. Michael Dunn, AP Hazen, Edwin Mares, Robert K Meyer, Graham Priest, Greg Restall, David Ripley, John Slaney, et al. On the ternary relation and conditionality. Journal of philosophical logic, 41(3):595-612, 2012.

[32] Patrick Blackburn, Maarten de Rijke, and Yde Venema. Modal Logic, volume 53 of Cambridge Tracts in Computer Science. Cambridge University Press, 2001.

[33] Alexander Bochman. Dynamic causal calculus. In KR, 2014.

[34] John P Burgess. Quick completeness proofs for some logics of conditionals. Notre Dame Journal of Formal Logic, 22(1):76-84, 1981. 
[35] Alonzo Church. Application of recursive arithmetic to the problem of circuit synthesis. In Summaries of the Summer Institute of Symbolic Logic, volume 1, pages 3-50, Cornell University, 1957.

[36] Luis Fariñas del Cerro, Andreas Herzig, and Jérôme Mengin, editors. Logics in Artificial Intelligence - 13th European Conference, JELIA 2012, Toulouse, France, September 2628, 2012. Proceedings, volume 7519 of Lecture Notes in Computer Science. Springer, 2012.

[37] J. Michael Dunn and Greg Restall. Relevance logic. Handbook of philosophical logic, 6:1-128, 2002.

[38] Ronald Fagin, Joseph Halpern, Yoram Moses, and Moshe Vardi. Reasoning about knowledge. MIT Press, 1995.

[39] Raul Fervari. Relation-Changing Modal Logics. PhD thesis, Universidad Nacional de Córdoba, 2014.

[40] Melvin Fitting. Modal proof theory. In F. Wolter J. van Benthem, P. Blackburn, editor, Handbook of Modal Logic, pages 85-138. Elsevier, 2007.

[41] Tim French, Wiebe van der Hoek, Petar Iliev, and Barteld Kooi. On the succinctness of some modal logics. Artificial Intelligence, 197(0):56 - 85, 2013.

[42] Sabine Frittella, Giuseppe Greco, Alexander Kurz, Alessandra Palmigiano, and Vlasta Sikimić. Multi-type display calculus for dynamic epistemic logic. Journal of Logic and Computation, 2014.

[43] Sabine Frittella, Giuseppe Greco, Alexander Kurz, Alessandra Palmigiano, and Vlasta Sikimić. A proof-theoretic semantic analysis of dynamic epistemic logic. Journal of Logic and Computation, 2014.

[44] Dov M Gabbay, Christopher John Hogger, John Alan Robinson, J Siekmann, and Donald Nute, editors. Handbook of logic in artificial intelligence and logic programming, volume Nonmonotonic reasoning and uncertain reasoning (Volume 3). Clarendon Press, 1998.

[45] Peter Gärdenfors. Knowledge in Flux (Modeling the Dynamics of Epistemic States). Bradford/MIT Press, Cambridge, Massachusetts, 1988.

[46] Peter Gärdenfors. Belief revision and nonmonotonic logic: Two sides of the same coin? In Logics in AI, pages 52-54. Springer, 1991.

[47] Gerhard Gentzen. Untersuchungen über das logische schließen. i. Mathematische zeitschrift, 39(1):176-210, 1935.

[48] Laura Giordano, Valentina Gliozzi, Nicola Olivetti, Gian Luca Pozzato, and Camilla B Schwind. Non-classical logics for knowledge representation and reasoning. Intelligenza Artificiale, 5(1):127-131, 2011. 
[49] Laura Giordano and Camilla Schwind. Conditional logic of actions and causation. Artificial Intelligence, 157(1):239-279, 2004.

[50] Patrick Girard, Jeremy Seligman, and Fenrong Liu. General dynamic dynamic logic. In Thomas Bolander, Torben Braüner, Silvio Ghilardi, and Lawrence S. Moss, editors, Advances in Modal Logic, pages 239-260. College Publications, 2012.

[51] J. Halpern and Y. Moses. Knowledge and common knowledge in a distributed environment. Journal of the ACM, 37(3):549-587, 1990.

[52] Jaakko Hintikka. Knowledge and Belief, An Introduction to the Logic of the Two Notions. Cornell University Press, Ithaca and London, 1962.

[53] C.A.R. Hoare. An axiomatic basis for computer programming. Communications of the ACM, 12(10):567-580, 1969.

[54] Tomohiro Hoshi. Epistemic dynamics and protocol information. PhD thesis, Stanford University, 2009.

[55] Barteld Kooi. Expressivity and completeness for public update logics via reduction axioms. Journal of Applied Non-Classical Logics, 17(2):231-253, 2007.

[56] Barteld Kooi and Bryan Renne. Arrow update logic. The Review of Symbolic Logic, 4(04):536-559, 2011.

[57] Orna Kupferman and Moshe Y. Vardi. Church's problem revisited. Bulletin of Symbolic Logic, 5(2):245-263, 1999.

[58] Natasha Kurtonina. Frames and Labels. A Modal Analysis of Categorial Deduction. PhD thesis, Ph. D. Thesis, Onderzoeksinstituut voor Taal en Spraak, University of Utrecht \& Institute for Logic, Language and Computation, University of Amsterdam, 1995.

[59] Joachim Lambek. The mathematics of sentence structure. American mathematical monthly, 65:154-170, 1958.

[60] Joachim Lambek. On the calculus of syntactic types. In R. Jakobson, editor, Structure of language and its mathematical aspects, pages 166-178. American Mathematical Society, Providence, 1961.

[61] Fenrong Liu. Changing for the Better: Preference Dynamics and Agent Diversity. $\mathrm{PhD}$ thesis, ILLC, University of Amsterdam, 2008.

[62] David Makinson. Bridges from classical to nonmonotonic logic. King's College, 2005.

[63] David Makinson and Peter Gärdenfors. Relations between the logic of theory change and nonmonotonic logic. In André Fuhrmann and Michael Morreau, editors, The Logic of Theory Change, volume 465 of Lecture Notes in Computer Science, pages 185-205. Springer, 1989. 
[64] Edwin D Mares. Relevant logic and the theory of information. Synthese, 109(3):345-360, 1996.

[65] Edwin D. Mares and Robert K. Meyer. The Blackwell guide to philosophical logic, chapter Relevant Logics. Wiley-Blackwell, 2001.

[66] Maricarmen Martinez and Sebastian Sequoiah-Grayson. Logic and information. In Edward N. Zalta, editor, The Stanford Encyclopedia of Philosophy. Summer 2014 edition, 2014.

[67] Elliott Mendelson. Introduction to mathematical logic. CRC press, 1997.

[68] Michael Moortgat. Categorial type logics. In Johan van Benthem and Alice ter Meulen, editors, Handbook of logic and language, chapter 2, pages 95 - 180. Elsevier, 2010.

[69] Richard Moot and Christian Retoré. A logic for categorial grammars: Lambek's syntactic calculus. In The Logic of Categorial Grammars, volume 6850 of Lecture Notes in Computer Science, pages 23-63. Springer Berlin Heidelberg, 2012.

[70] Reinhard Muskens, Johan van Benthem, and Albert Visser. Handbook of logic and language, chapter Dynamics, pages 607-670. Elsevier, 2011.

[71] Sara Negri and Nicola Olivetti. A sequent calculus for preferential conditional logic based on neighbourhood semantics. In Nivelle [73], pages 115-134.

[72] Sara Negri and Giorgio Sbardolini. Proof analysis for Lewis counterfactuals. The Review of Symbolic Logic, FirstView:1-32, 122015.

[73] Hans De Nivelle, editor. Automated Reasoning with Analytic Tableaux and Related Methods - 24th International Conference, TABLEAUX 2015, Wroctaw, Poland, September 2124, 2015. Proceedings, volume 9323 of Lecture Notes in Computer Science. Springer, 2015.

[74] Donald Nute and Charles B Cross. Conditional logic. In Dov Gabbay and F. Guenthner, editors, Handbook of philosophical logic, volume 4, pages 1-98. Kluwer Academic Pub, 2001.

[75] Nicola Olivetti and Gian Luca Pozzato. A standard internal calculus for lewis' counterfactual logics. In Nivelle [73], pages 270-286.

[76] Nicola Olivetti, Gian Luca Pozzato, and Camilla Schwind. A sequent calculus and a theorem prover for standard conditional logics. ACM Trans. Comput. Log., 8(4), 2007.

[77] Hiroakira Ono. Proof-theoretic methods in nonclassical logic -an introduction. In Masako Takahashi, Mitsuhiro Okada, and Mariangiola Dezani-Ciancaglini, editors, Theories of Types and Proofs, volume Volume 2 of MSJ Memoirs, pages 207-254. The Mathematical Society of Japan, Tokyo, Japan, 1998. 
[78] John Perry and David Israel. What is information? Information, Language, and Cognition, 1, 1990.

[79] Jan Plaza. Logics of public communcations. In M. L. Emrich, M. Z. Pfeifer, M. Hadzikadic, and Z. W. Ras, editors, Proceedings of the 4th International Symposium on Methodologies for Intelligent Systems, pages 201-216, 1989.

[80] Francesca Poggiolesi. Gentzen calculi for modal propositional logic, volume 32. Springer, 2010.

[81] Francesca Poggiolesi and Greg Restall. Interpreting and applying proof theories for modal logic. In New Waves in Philosophical Logic. Palgrave Macmillan, 2012.

[82] Vaughan Pratt. Semantical considerations on floyd-hoare logic. In Proceedings of the 17th IEEE Symposium on the Foundations of Computer Science, pages 109-121, 1976.

[83] Frank P. Ramsey. General propositions and causality. In H.A. Mellor, editor, Philosophical Papers. Cambridge University Press, Cambridge, 1929.

[84] Raymond Reiter. Knowledge in Action: Logical Foundations for Specifying and Implementing Dynamical Systems. MIT Press, 2001.

[85] Greg Restall. Information flow and relevant logics. In Logic, Language and Computation: The 1994 Moraga Proceedings. CSLI, pages 463-477. csli Publications, 1996.

[86] Greg Restall. An Introduction to Substructural Logics. Routledge, 2000.

[87] Greg Restall. Relevant and substructural logics. Handbook of the History of Logic, 7:289398, 2006.

[88] Richard Routley and Robert K Meyer. The semantics of entailment-ii. Journal of Philosophical Logic, 1(1):53-73, 1972.

[89] Richard Routley and Robert K Meyer. The semantics of entailment-iii. Journal of philosophical logic, 1(2):192-208, 1972.

[90] Richard Routley and Robert K Meyer. The semantics of entailment. Studies in Logic and the Foundations of Mathematics, 68:199-243, 1973.

[91] Richard Routley, Val Plumwood, and Robert K Meyer. Relevant logics and their rivals. Ridgeview Publishing Company, 1982.

[92] Murray Shanahan. Solving the Frame Problem. MIT press, Cambridge, Massachusetts, 1997.

[93] Alasdair Urquhart. Completeness of weak implication. Theoria, 37(3):274-282, 1971.

[94] Alasdair Urquhart. A general theory of implication. Journal of Symbolic Logic, 37(443):270, 1972. 
[95] Alasdair Urquhart. Semantics for relevant logics. Journal of Symbolic Logic, pages 159169, 1972.

[96] Johan van Benthem. General dynamics. Theoretical Linguistics, 17(1-3):159-202, 1991.

[97] Johan van Benthem. Exploring logical dynamics. CSLI publications Stanford, 1996.

[98] Johan van Benthem. Meaning: the Dynamic Turn, chapter Structural Properties of Dynamic Reasoning, pages 15-31. Elsevier, Amsterdam, 2003.

[99] Johan van Benthem. Dynamic logic for belief revision. Journal of Applied Non-Classical Logics, 17(2):129-155, 2007.

[100] Johan van Benthem. Inference in action. Publications de l'Institut MathématiqueNouvelle Série, 82(96):3-16, 2007.

[101] Johan van Benthem. Logical dynamics meets logical pluralism? The Australasian Journal of Logic, 6:182-209, 2008.

[102] Johan van Benthem. Modal logic for open minds. CSLI publications, 2010.

[103] Johan van Benthem. Logical Dynamics of Information and Interaction. Cambridge University Press, 2011.

[104] Johan van Benthem. McCarthy variations in a modal key. Artificial intelligence, 175(1):428-439, 2011.

[105] Johan van Benthem, Jelle Gerbrandy, Tomohiro Hoshi, and Eric Pacuit. Merging frameworks for interaction. Journal of Philosophical Logic, 38(5):491-526, 2009.

[106] Johan van Benthem, Jelle Gerbrandy, and Barteld Kooi. Dynamic update with probability. Studia Logica, 93(1):67-96, 2009.

[107] Johan van Benthem and Barteld Kooi. Reduction axioms for epistemic actions. In R. Schmidt, I. Pratt-Hartmann, M. Reynolds, and H. Wansing, editors, AiML-2004: Advances in Modal Logic, number UMCS-04-9-1 in Technical Report Series, pages 197211, University of Manchester, 2004.

[108] Ron van der Meyden and Moshe Y. Vardi. Synthesis from knowledge-based specifications (extended abstract). In Davide Sangiorgi and Robert de Simone, editors, CONCUR, volume 1466 of Lecture Notes in Computer Science, pages 34-49. Springer, 1998.

[109] Hans van Ditmarsch. Prolegomena to dynamic logic for belief revision. Synthese, 147:229-275, 2005.

[110] Hans van Ditmarsch, Wiebe van der Hoek, and Barteld Kooi. Dynamic Epistemic Logic, volume 337 of Synthese library. Springer, 2007. 
[111] Hans P. van Ditmarsch, Sujata Ghosh, Rineke Verbrugge, and Yanjing Wang. Hidden protocols: Modifying our expectations in an evolving world. Artif. Intell., 208:18-40, 2014.

[112] Hans P. van Ditmarsch, Andreas Herzig, and Tiago De Lima. From situation calculus to dynamic epistemic logic. Journal of Logic and Computation, 21(2):179-204, 2009.

[113] Jan van Eijck. Reducing dynamic epistemic logic to PDL by program transformation. Technical Report SEN-E0423, CWI, 2004.

[114] Jan van Eijck, Floor Sietsma, and Yanjing Wang. Composing models. Journal of Applied Non-Classical Logics, 21(3-4):397-425, 2011.

[115] Yanjing Wang. Epistemic Modelling and Protocol Dynamics. PhD thesis, Universiteit van Amsterdam, 2010.

[116] Heinrich Wansing. Sequent calculi for normal modal propositional logics. Journal of Logic and Computation, 4(2):125-142, 1994.

\section{A Completeness Proof of UL*}

The proof follows the same lines as the completeness proof of UL, so we only sketch it. We mention the parts which are different and provide the missing proofs.

Definition 31 ((maximal) $U L^{*}\left(C_{\varphi}\right)$-consistent set of $\mathcal{S}^{\mathbb{P} *}$ and $\left.\mathcal{S}^{\mathbb{A} *}\right)$. Let $\mathrm{C}_{\varphi} \subseteq \operatorname{Con}_{\varphi}$.

- An $U L^{*}\left(C_{\varphi}\right)$-consistent set of $\mathcal{S}^{\mathbb{P} *}$ is a set $\Gamma$ of $\mathcal{L}\left(\mathbb{P}, C_{\varphi}, \mathbb{P}\right)$-structures of $\mathcal{S}^{\mathbb{P} *}$ such that there are no $X_{1}, \ldots, X_{n} \in \Gamma$ such that $X_{1}, \ldots, X_{n} \vdash$.

- A maximal $U L^{*}\left(C_{\varphi}\right)$-consistent set of $\mathcal{S}^{\mathbb{P} *}$ is a $\operatorname{UL}^{*}\left(\mathrm{C}_{\varphi}\right)$-consistent set $\Gamma$ of $\mathcal{S}^{\mathbb{P} *}$ such that there is no $X \in \mathcal{S}^{\mathbb{P} *}$ such that $X \notin \Gamma$ and $\Gamma \cup\{X\}$ is $\mathrm{UL}^{*}\left(\mathrm{C}_{\varphi}\right)$-consistent.

Similar definitions hold for the $\mathcal{L}_{\mathrm{DEL}}\left(\mathrm{C}_{\varphi}\right)$-structures of $\mathcal{S}^{\mathbb{A} *}$.

Then, like in [12], we can prove the same preliminary results and the same Cut Lemma and Lindenbaum Lemma. For the sake of completeness, we recall them below:

Lemma 15 (Cut Lemma). Let $\Gamma$ be a maximal UL-consistent set of $\mathcal{S}^{\mathbb{P} *}$ or $\mathcal{S}^{\mathbb{A} *}$. For all $X_{1}, \ldots, X_{k} \in \Gamma$, all $\varphi \in \mathcal{L}_{U L^{*}\left(C_{\varphi}\right)}^{D}$, if $X_{1}, \ldots, X_{k} \vdash \varphi$ then $\varphi \in \Gamma$.

Lemma 16. Any UL-consistent set over $\mathcal{S}^{\mathbb{P} *}\left(\mathcal{S}^{\mathbb{A} *}\right)$ can be extended into a maximal UL-consistent set over $\mathcal{S}^{\mathbb{P} *}\left(\right.$ resp. $\left.\mathcal{S}^{\mathbb{A} *}\right)$.

The definition of the canonical update model associated to $\mathrm{UL}^{*}$ is however different:

Definition 32 (Canonical update model). The canonical update model associated to $U L^{*}$ is the tuple $\mathcal{M}^{c}:=\left(\mathcal{P}^{c}, \mathcal{R}_{\square_{1}}^{c}, \ldots, \mathcal{R}_{\square_{m}}^{c}, \mathcal{R}_{\diamond_{1}}^{c}, \ldots, \mathcal{R}_{\diamond_{m}}^{c}, \mathcal{R}_{\subset_{1}}^{c}, \mathcal{R}_{\subset_{2}}^{c}, \mathcal{R}_{\triangle_{3}}^{c}, \mathcal{R}_{\supset_{1}}^{c}, \mathcal{R}_{\supset_{2}}^{c}, \mathcal{R}_{\supset_{3}}^{c}, \mathcal{R}_{\otimes_{1}}^{c}, \mathcal{R}_{\otimes_{2}}^{c}, \mathcal{R}_{\otimes_{3}}^{c}, \mathcal{I}^{c}\right)$ defined as follows: 
- $\mathcal{P}^{c}:=\left(P^{c},=\right)$ where $P^{c}$ is the disjoint union of the set $\mathcal{E}^{\mathbb{P}}$ of all maximal UL-consistent sets of $\mathcal{S}^{\mathbb{P} *}$ and the set $\mathcal{E}^{\mathbb{A}}$ of all maximal UL-consistent sets of $\mathcal{S}^{\mathbb{A} *}$;

- for all $\Gamma, \Gamma^{\prime}, \Gamma^{\prime \prime} \in \mathcal{E}^{\mathbb{P}}$, all $\Gamma^{\prime} \in \mathcal{E}^{\mathbb{A}}$, all $j=1, \ldots, m$,

$\begin{array}{lll}\left(\Gamma, \Gamma^{\prime}\right) \in \mathcal{R}_{\square_{j}}^{c} & \text { iff } & \text { for all } \square_{j} \varphi \in \Gamma, \text { we have that } \varphi \in \Gamma^{\prime} \\ \left(\Gamma, \Gamma^{\prime}\right) \in \mathcal{R}_{\diamond_{j}^{-}}^{c} & \text { iff } & \text { for all } \varphi \in \Gamma^{\prime}, \text { we have that } \diamond_{j}^{-} \varphi \in \Gamma \\ & & \\ \left(\Gamma, \Gamma^{\prime}, \Gamma^{\prime \prime}\right) \in \mathcal{R}_{\supset_{1}}^{c} & \text { iff } & \text { for all } \chi \supset_{1} \varphi \in \Gamma, \text { if } \chi \in \Gamma^{\prime} \text { then } \varphi \in \Gamma^{\prime \prime} \\ \left(\Gamma, \Gamma^{\prime}, \Gamma^{\prime \prime}\right) \in \mathcal{R}_{\supset_{2}}^{c} & \text { iff } & \text { for all } \psi \supset_{2} \varphi \in \Gamma^{\prime}, \text { if } \psi \in \Gamma^{\prime \prime} \text { then } \varphi \in \Gamma \\ \left(\Gamma, \Gamma^{\prime}, \Gamma^{\prime \prime}\right) \in \mathcal{R}_{\supset_{3}}^{c} & \text { iff } & \text { for all } \varphi \supset_{3} \chi \in \Gamma^{\prime \prime}, \text { if } \varphi \in \Gamma \text { then } \chi \in \Gamma^{\prime} \\ \left(\Gamma, \Gamma^{\prime}, \Gamma^{\prime \prime}\right) \in \mathcal{R}_{\subset_{1}}^{c} & \text { iff } \quad \text { for all } \chi \subset_{1} \varphi \in \Gamma^{\prime \prime}, \text { if } \varphi \in \Gamma^{\prime \prime} \text { then } \chi \in \Gamma^{\prime} \\ \left(\Gamma, \Gamma^{\prime}, \Gamma^{\prime \prime}\right) \in \mathcal{R}_{\subset_{2}}^{c} & \text { iff } \quad \text { for all } \psi \subset_{2} \varphi \in \Gamma^{\prime}, \text { if } \varphi \in \Gamma \text { then } \psi \in \Gamma^{\prime \prime} \\ \left(\Gamma, \Gamma^{\prime}, \Gamma^{\prime \prime}\right) \in \mathcal{R}_{\subset_{3}}^{c} & \text { iff } \quad \text { for all } \varphi \subset_{3} \chi \in \Gamma^{\prime \prime}, \text { if } \chi \in \Gamma^{\prime} \text { then } \varphi \in \Gamma \\ \left(\Gamma, \Gamma^{\prime}, \Gamma^{\prime \prime}\right) \in \mathcal{R}_{\otimes_{1}}^{c} & \text { iff } \quad \text { for all } \psi \in \Gamma^{\prime \prime} \text { and all } \chi \in \Gamma^{\prime}, \chi \otimes_{1} \psi \in \Gamma \\ \left(\Gamma, \Gamma^{\prime}, \Gamma^{\prime \prime}\right) \in \mathcal{R}_{\otimes_{2}}^{c} & \text { iff } \quad \text { for all } \psi \in \Gamma^{\prime \prime} \text { and all } \varphi \in \Gamma, \psi \otimes_{2} \varphi \in \Gamma^{\prime} \\ \left(\Gamma, \Gamma^{\prime}, \Gamma^{\prime \prime}\right) \in \mathcal{R}_{\otimes_{3}}^{c} & \text { iff } \quad \text { for all } \varphi \in \Gamma \text { and all } \chi \in \Gamma^{\prime}, \varphi \otimes_{3} \chi \in \Gamma^{\prime \prime}\end{array}$

- for all $p \in \mathbb{P}$, all $p_{\pi} \in \mathbb{A}$,

$$
\begin{array}{lll}
p \in \mathcal{I}^{c}(\Gamma) & \text { iff } & p \in \Gamma \\
p_{\pi} \in \mathcal{I}^{c}\left(\Gamma^{\prime}\right) & \text { iff } & p_{\pi} \in \Gamma^{\prime}
\end{array}
$$

Lemma 17. The following hold:

- $\mathcal{R}_{\square_{j}}^{c}=\mathcal{R}_{\diamond_{j}}^{c}$ for all $j \in\{1, \ldots, m\}$;

- $\mathcal{R}_{\subset_{1}}^{c}=\mathcal{R}_{\subset_{2}}^{c}=\mathcal{R}_{\subset_{3}}^{c}=\mathcal{R}_{\supset_{1}}^{c}=\mathcal{R}_{\supset_{2}}^{c}=\mathcal{R}_{\supset_{3}}^{c}=\mathcal{R}_{\otimes_{1}}^{c}=\mathcal{R}_{\otimes_{2}}^{c}=\mathcal{R}_{\otimes_{3}}^{c}$.

Proof. We prove the first item, that is $\mathcal{R}_{\square_{j}}^{c}=\mathcal{R}_{\diamond_{j}}^{c}$. We first show that $\mathcal{R}_{\square_{j}}^{c} \subseteq \mathcal{R}_{\diamond_{j}}^{c}$. Let $\left(\Gamma, \Gamma^{\prime}\right) \in \mathcal{R}_{\diamond_{j}}^{c}$ and let $\square_{j} \varphi \in \Gamma$. We mush show that $\varphi \in \Gamma^{\prime}$. Assume that $\varphi \notin \Gamma^{\prime}$. Then, because $\Gamma^{\prime}$ is a maximal UL-consistent set, we have that $\neg \varphi \in \Gamma^{\prime}$. Then, by definition of $\mathcal{R}_{\diamond_{j}}^{c}$, we have that $\diamond_{j} \neg \varphi \in \Gamma(*)$. However, one can prove that $\square_{j} \varphi \vdash \neg \diamond_{j} \neg \varphi$. Therefore, because $\square_{j} \varphi \in \Gamma$, we also have that $\neg \diamond_{j} \neg \varphi \in \Gamma(* *)$. Putting $(*)$ and (**) altogether, we obtain that $\Gamma$ is not UL-consistent, which is impossible. The proof of the other inclusion $\mathcal{R}_{\diamond_{j}}^{c} \subseteq \mathcal{R}_{\square_{j}}^{c}$ is similar and relies on the fact that $\diamond_{j} \varphi \vdash \neg \square_{j} \neg \varphi$.

Now, we prove the second item. The proofs that $\mathcal{R}_{\supset_{1}}^{c}=\mathcal{R}_{\subset_{2}}^{c}=\mathcal{R}_{\otimes_{3}}^{c}$, that $\mathcal{R}_{\subset_{3}}^{c}=\mathcal{R}_{\supset_{2}}^{c}=$ $\mathcal{R}_{\otimes_{1}}^{c}$ and that $\mathcal{R}_{\supset_{3}}^{c}=\mathcal{R}_{\subset_{1}}^{c}=\mathcal{R}_{\otimes_{2}}^{c}$ are similar to the proof of Lemma 11.25 of [86]. In fact, we only prove $\mathcal{R}_{\otimes_{3}}^{c}=\mathcal{R}_{\otimes_{1}}^{c}$, the proof that $\mathcal{R}_{\otimes_{1}}^{c}=\mathcal{R}_{\otimes_{2}}^{c}$ is similar. First, we prove that $\mathcal{R}_{\otimes_{3}}^{c} \subseteq$ $\mathcal{R}_{\otimes_{1}}^{c}$. Let $\left(\Gamma, \Gamma^{\prime}, \Gamma^{\prime \prime}\right) \in \mathcal{R}_{\otimes_{3}}^{c}$, we are going to show that $\left(\Gamma, \Gamma^{\prime}, \Gamma^{\prime \prime}\right) \in \mathcal{R}_{\otimes_{1}}^{c}$. Let $\psi \in \Gamma^{\prime \prime}$ and $\chi \in \Gamma^{\prime}$. We are going to prove that $\chi \otimes_{1} \psi \in \Gamma$. Assume towards a contradiction that $\chi \otimes_{1} \psi \notin \Gamma$. Then, $\neg\left(\chi \otimes_{1} \psi\right) \in \Gamma$, because $\Gamma$ is a maximal consistent set. However, one can prove using,$_{2} /,_{3}$ that $\neg\left(\chi \otimes_{1} \psi\right) \otimes_{3} \chi \vdash \neg \psi$. Now, because $\left(\Gamma, \Gamma^{\prime}, \Gamma^{\prime \prime}\right) \in \mathcal{R}_{\otimes_{3}}^{c}$ we have that 
$\neg\left(\chi \otimes_{1} \psi\right) \otimes_{3} \chi \in \Gamma^{\prime}$. Therefore, $\neg \psi \in \Gamma^{\prime \prime}$. This contradicts the fact that $\psi \in \Gamma^{\prime \prime}$. Thus, $\chi \otimes_{1} \psi \in \Gamma$ and $\left(\Gamma, \Gamma^{\prime}, \Gamma^{\prime \prime}\right) \in \mathcal{R}_{\otimes_{1}}^{c}$. Hence, $\mathcal{R}_{\otimes_{3}}^{c} \subseteq \mathcal{R}_{\otimes_{1}}^{c}$. Reciprocally, let $\left(\Gamma, \Gamma^{\prime}, \Gamma^{\prime \prime}\right) \in \mathcal{R}_{\otimes_{1}}^{c}$. We are going to show that $\left(\Gamma, \Gamma^{\prime}, \Gamma^{\prime \prime}\right) \in \mathcal{R}_{\otimes_{3}}^{c}$. Let $\varphi \in \Gamma$ and $\chi \in \Gamma^{\prime}$, we are going to show that $\varphi \otimes_{3} \chi \in \Gamma^{\prime \prime}$. Assume towards a contradiction that $\varphi \otimes_{3} \chi \notin \Gamma^{\prime \prime}$. Then, $\neg\left(\varphi \otimes_{3} \chi\right) \in \Gamma^{\prime \prime}$. Now, because $\left(\Gamma, \Gamma^{\prime}, \Gamma^{\prime \prime}\right) \in \mathcal{R}_{\otimes_{1}}^{c}$ and $\chi \in \Gamma^{\prime}$, we have that $\chi \otimes_{1} \neg\left(\varphi \otimes_{3} \chi\right) \in \Gamma$ by definition of $\mathcal{R}_{\otimes_{1}}^{c}$. Therefore, $\neg \varphi \in \Gamma$ because one can prove using ,,$_{2} /,{ }_{3}$ that $\chi \otimes_{1} \neg\left(\varphi \otimes_{3} \chi\right) \vdash \neg \varphi$. This is impossible by assumption. Thus, $\varphi \otimes_{3} \chi \in \Gamma^{\prime \prime}$ and $\left(\Gamma, \Gamma^{\prime}, \Gamma^{\prime \prime}\right) \in \mathcal{R}_{\otimes_{3}}^{c}$. Hence, $\mathcal{R}_{\otimes_{1}}^{c} \subseteq \mathcal{R}_{\otimes_{3}}^{c}$. So, finally, $\mathcal{R}_{\otimes_{1}}^{c}=\mathcal{R}_{\otimes_{3}}^{c}$.

Hence, from now on, we will use the following two notations: $\mathcal{R}_{j}^{c}:=\mathcal{R}_{\square_{j}}^{c}=\mathcal{R}_{\diamond_{j}}^{c}$ for all $j \in\{1, \ldots, m\}$ and $\mathcal{R}^{c}:=\mathcal{R}_{\supset_{1}}^{c}=\mathcal{R}_{\subset_{2}}^{c}=\mathcal{R}_{\subset_{3}}^{c}=\mathcal{R}_{\supset_{2}}^{c}=\mathcal{R}_{\supset_{3}}^{c}=\mathcal{R}_{\subset_{1}}^{c^{j}}=\mathcal{R}_{\otimes_{1}}^{c}=$ $\mathcal{R}_{\otimes_{2}}^{c}=\mathcal{R}_{\otimes_{3}}^{c}$. With these notations, the canonical model associated to UL is the update model $\mathcal{M}^{c}=\left(\mathcal{P}^{c}, \mathcal{R}_{1}^{c}, \ldots, \mathcal{R}_{m}^{c}, \mathcal{R}^{c}, \mathcal{I}^{c}\right)$.

Lemma 18 (Truth lemma). For all $\mathcal{L}_{D E L}$-structure $X$, for all maximal consistent sets $\Gamma$, we have that

$$
\mathcal{M}^{c}, \Gamma \|^{A} X \quad \text { iff } \quad X \in \Gamma .
$$

Proof. The proof is similar to the proof of Truth Lemma of [12]. We only deal with the cases which are not dealt with in the Truth Lemma of [12] or that are different. That is, we only prove it for the cases $\neg, \supset_{1}$ and $\subset_{2}$, the cases $\varphi \subset_{3} \chi, \psi \supset_{2} \varphi$ and $\varphi \supset_{3} \chi, \chi \subset_{1} \psi$ are dealt with similarly and the proofs can be easily adapted. For the case $\subset_{2}$, we will use the following fact:

$$
\psi \subset_{\mathrm{i}} \varphi_{1}, \ldots, \psi \subset_{\mathrm{i}} \varphi_{n} \vdash \psi \subset_{\mathrm{i}}\left(\varphi_{1} \vee \ldots \vee \varphi_{n}\right)
$$

We prove Expression (19] as follows. By iterated application of $\vee_{A}$ to $\varphi_{1} \vdash \varphi_{1}, \ldots, \varphi_{n-1} \vdash \varphi_{n-1}$ and $\varphi_{n} \vdash \varphi_{n}$, we obtain that $\varphi_{1} \vee \ldots \vee \varphi_{n} \vdash \varphi_{1}, \ldots, \varphi_{n}$. Then, by application of $\subset_{A}^{\mathrm{i}}$ and $\subset_{K}^{\mathrm{i}}$ to $\varphi_{1} \vee \ldots \vee \varphi_{n} \vdash \varphi_{1}, \ldots, \varphi_{n}$ and $\psi \vdash \psi$, we have that $\left(\varphi_{1} \vee \ldots \vee \varphi_{n}\right){ }_{,_{3}}\left(\psi \subset_{2} \varphi_{1}, \ldots, \psi \subset_{2}\right.$ $\left.\varphi_{n}\right) \vdash \psi$. Finally, by $\subset_{K}^{2}$, we obtain that $\psi \subset_{\mathrm{i}} \varphi_{1}, \ldots, \psi \subset_{\mathrm{i}} \varphi_{n} \vdash \psi \subset_{\mathrm{i}}\left(\varphi_{1} \vee \ldots \vee \varphi_{n}\right)$.

- $\neg \psi$ :

Assume that $\neg \psi \in \Gamma$. If $\psi \in \Gamma$, then because $\psi, \neg \psi \vdash$ and $\psi, \neg \psi \in \Gamma, \Gamma$ would not be $\mathrm{UL}^{*}$-consistent. Therefore, it is not the case that $\psi \in \Gamma$, that is, by Induction Hypothesis, it is not the case that $\mathcal{M}^{c}, \Gamma \|^{A} \psi$. That is, $\mathcal{M}^{c}, \Gamma \|^{A} \neg \psi$. For the other direction, if $\mathcal{M}^{c}, \Gamma \|^{A} \neg \psi$, then it is not the case that $\mathcal{M}^{c}, \Gamma \|^{A} \psi$. So, by Induction Hypothesis, $\psi \notin \Gamma$. If $\neg \psi \notin \Gamma$ then we both have that $\psi \notin \Gamma$ and $\neg \psi \notin \Gamma$. Then, $\Gamma \cup\{\psi\}$ and $\Gamma \cup\{\neg \psi\}$ are not $\mathrm{UL}^{*}$-consistent because $\Gamma$ is a maximal $\mathrm{UL}^{*}$-consistent set. So, there are $\varphi_{1}^{\prime}, \ldots, \varphi_{n}^{\prime} \in$ $\Gamma, \psi_{1}^{\prime}, \ldots, \psi_{m}^{\prime} \in \Gamma$ such that $\varphi_{1}^{\prime}, \ldots, \varphi_{n}^{\prime}, \psi \vdash$ and $\psi_{1}^{\prime}, \ldots, \psi_{m}^{\prime}, \neg \psi \vdash$. Thus, by the Weakening rule $\mathrm{K}$, we have that $\varphi_{1}^{\prime}, \ldots, \varphi_{n}^{\prime}, \psi_{1}^{\prime}, \ldots, \psi_{m}^{\prime}, \psi \vdash$ and $\varphi_{1}^{\prime}, \ldots, \varphi_{n}^{\prime}, \psi_{1}^{\prime}, \ldots, \psi_{m}^{\prime}, \neg \psi \vdash$. Therefore, we have that $\varphi_{1}^{\prime}, \ldots, \varphi_{n}^{\prime}, \psi_{1}^{\prime}, \ldots, \psi_{m}^{\prime}, \psi \vee \neg \psi \vdash$ by rule $\vee_{L}$. But, we have that $\varphi_{1}^{\prime}, \ldots, \varphi_{n}^{\prime}, \psi_{1}^{\prime}, \ldots, \psi_{m}^{\prime} \in \Gamma$ and $\psi \vee \neg \psi \in \Gamma$, by application of the Cut Lemma (since $\vdash \psi \vee \neg \psi$ ). So, $\Gamma$ is not $\mathrm{UL}^{*}$-consistent, which is impossible. So, $\neg \psi \in \Gamma$, which proves the other direction. 
- $\chi \supset_{1} \psi$ :

Assume that $\chi \supset_{1} \psi \in \Gamma$. Then, for all $\Gamma^{\prime}, \Gamma^{\prime \prime}$ such that $\left(\Gamma, \Gamma^{\prime}, \Gamma^{\prime \prime}\right) \in \mathcal{R}^{c}$, if $\chi \in \Gamma^{\prime}$ then $\psi \in \Gamma^{\prime \prime}$. That is, for all $\Gamma^{\prime}, \Gamma^{\prime \prime}$ such that $\left(\Gamma, \Gamma^{\prime}, \Gamma^{\prime \prime}\right) \in \mathcal{R}^{c}$, if $\mathcal{M}^{c}, \Gamma^{\prime} \Vdash \chi$ then $\mathcal{M}^{c}, \Gamma^{\prime \prime} \Vdash \psi$ by Induction Hypothesis. That is, $\mathcal{M}^{c}, \Gamma \| \chi \supset_{1} \psi$.

Assume that $\mathcal{M}^{c}, \Gamma \Vdash \chi \supset_{1} \psi$ and assume towards a contradiction that $\chi \supset_{1} \psi \notin \Gamma$.

1. Assume that $S:=\left\{\varphi \mid \chi \supset_{1} \varphi \in \Gamma\right\} \cup\{\neg \psi\}$ is not $\cup L^{*}$-consistent. Then, there are $\varphi_{1}, \ldots, \varphi_{n} \in S$ such that $\neg \psi, \varphi_{1}, \ldots, \varphi_{n} \vdash$. Hence, $\varphi_{1}, \ldots, \varphi_{n} \vdash \neg \neg \psi$ by the rules $\neg_{R}$ and $\neg_{L}$. So, $\chi \supset_{1} \varphi_{1}, \ldots, \chi \supset_{1} \varphi_{n} \vdash \chi \supset_{1} \neg \neg \psi$ by application of $\supset_{L}$ and then $\supset_{R}$ to $\varphi_{1}, \ldots, \varphi_{n} \vdash \neg \neg \psi$ and $\chi \vdash \chi$. Then, by application of Lemma 15, we have that $\chi \supset_{1} \neg \neg \psi \in \Gamma$. Moreover, because we also have that $\chi \supset_{1} \neg \neg \psi \vdash \chi \supset_{1} \psi$, it holds that $\chi \supset_{1} \psi \in \Gamma$. This is impossible. Therefore, $S$ is $\mathrm{UL}^{*}$-consistent and by Lemma 16 it can be extended into a maximal consistent set called $\Gamma^{\prime \prime}$.

2. Now, let $S_{\chi}:=\{\chi\} \cup\left\{\neg \rho \mid\right.$ there is $\left.\psi \notin \Gamma^{\prime \prime}, \rho \supset_{1} \psi \in \Gamma\right\}$. Assume that $S_{\chi}$ is not $\mathrm{UL}^{*}$-consistent. Then, there are $\neg \rho_{1}, \ldots, \neg \rho_{n} \in S_{\chi}$ such that $\chi, \neg \rho_{1}, \ldots, \neg \rho_{n} \vdash$. So, $\chi \vdash \neg \neg \rho_{1}, \ldots, \neg \neg \rho_{n}$ by $\neg R$. Therefore, we have that $\neg \neg \rho_{1} \supset_{1} \bigvee_{i} \psi_{i}, \ldots, \neg \neg \rho_{n} \supset_{1}$ $\bigvee_{i} \psi_{i} \vdash \chi \supset_{1} \bigvee_{i} \psi_{i}(*)$ by application of rules $\supset_{L}^{1}$ and $\supset_{K}^{1}$. Moreover, we can easily prove that for all $i \in\{1, \ldots, n\}, \rho_{i} \supset_{1} \psi_{i} \vdash \neg \neg \rho_{i} \supset_{1} \bigvee_{i} \psi_{i}$ by rules $\supset_{L}^{1}$ and $\supset_{K}^{1}$. Therefore, by application of Lemma 15 , we have that $\neg \neg \rho_{i} \supset_{1} \bigvee \psi_{i} \in \Gamma$, because by definition of $S_{\chi}$ we have that $\rho_{i} \supset_{1} \psi_{i} \in \Gamma$ for all $i \in\{1, \ldots, n\}$. Then, again by application of Lemma 15 to $(*)$, we have that $\chi \supset_{1} \bigvee_{i} \psi_{i} \in \Gamma$. Hence, $\bigvee_{i} \psi_{i} \in S$ by definition of $S$. So, $\bigvee_{i} \psi_{i} \in \Gamma^{\prime \prime}$ by definition of $\Gamma^{\prime \prime}$. However, we can easily prove that for all $i$, it holds that $\bigvee_{i} \psi_{i}, \neg \psi_{1}, \ldots, \neg \psi_{n} \vdash$. This entails that $\Gamma^{\prime \prime}$ is not $\mathrm{UL}^{*}$-consistent, because for all $i, \neg \psi_{i} \in \Gamma^{\prime \prime}$ and $\bigvee_{i} \psi_{i} \in \Gamma^{\prime \prime}$. We have reached a contradiction. Therefore our initial assumption was wrong and $S_{\chi}$ must be $\mathrm{UL}^{*}$-consistent. So, it can be extended into a maximal $U L^{*}$-consistent set called $\Gamma^{\prime}$.

Finally, we need to check that $\left(\Gamma, \Gamma^{\prime}, \Gamma^{\prime \prime}\right) \in \mathcal{R}^{c}$. Let $\rho \supset_{1} \varphi \in \Gamma$ and assume that $\rho \in \Gamma^{\prime}$ but $\varphi \notin \Gamma^{\prime \prime}$. Then, by definition of $\Gamma^{\prime}, \neg \rho \in \Gamma^{\prime}$, which contradicts the fact that $\rho \in \Gamma^{\prime}$. So, $\varphi \in \Gamma^{\prime \prime}$. Hence, there are $\left(\Gamma, \Gamma^{\prime}, \Gamma^{\prime \prime}\right) \in \mathcal{R}^{c}$ such that $\chi \in \Gamma^{\prime}$ and $\neg \psi \in \Gamma^{\prime \prime}$. So, there are $\left(\Gamma, \Gamma^{\prime}, \Gamma^{\prime \prime}\right) \in \mathcal{R}^{c}$ such that $\mathcal{M}^{c}, \Gamma^{\prime} \Vdash \chi$ and $\mathcal{M}^{c}, \Gamma^{\prime \prime} \Vdash \neg \psi$ by Induction Hypothesis. Therefore, it is not the case that $\mathcal{M}^{c}, \Gamma \Vdash \chi \supset_{1} \psi$, which contradicts our assumption. We have reached a contradiction, so, finally, $\chi \supset_{1} \psi \in \Gamma$.

- $\varphi \subset_{2} \psi$ :

Assume that $\psi \subset_{2} \varphi \in \Gamma^{\prime}$. Then, for all $\Gamma, \Gamma^{\prime \prime} \in \mathcal{M}^{c}$ such that $\left(\Gamma, \Gamma^{\prime}, \Gamma^{\prime \prime}\right) \in \mathcal{R}^{c}$, if $\psi \in \Gamma$ 
then $\varphi \in \Gamma^{\prime \prime}$. Then, for all $\Gamma, \Gamma^{\prime \prime} \in \mathcal{M}^{c}$ such that $\left(\Gamma, \Gamma^{\prime}, \Gamma^{\prime \prime}\right) \in \mathcal{R}^{c}$, if $\mathcal{M}^{c}, \Gamma \| \psi$ then $\mathcal{M}^{c}, \Gamma^{\prime \prime} \Vdash \varphi$. That is, $\mathcal{M}^{c}, \Gamma^{\prime} \| \psi \subset_{2} \varphi$.

Assume that $\mathcal{M}^{c}, \Gamma^{\prime} \| \psi \subset_{2} \varphi$. Assume towards a contradiction that $\psi \subset_{2} \varphi \notin \Gamma^{\prime}$.

1. Let $S_{f}:=\{\neg \varphi\} \cup\left\{\varphi \mid \psi \subset_{2} \varphi \in \Gamma^{\prime}\right\}$ and assume that $S_{f}$ is not $\mathrm{UL}^{*}$-consistent. Then, there are $\varphi_{1}, \ldots, \varphi_{n} \in S_{f}$ such that $\neg \varphi, \varphi_{1}, \ldots, \varphi_{n} \vdash$. So, $\varphi_{1}, \ldots, \varphi_{n} \vdash \neg \neg \varphi$ by the rules $\neg_{R}$ and $\neg_{L}$. Then, by application of $\subset_{L}$ and then $\subset_{R}$ to $\varphi_{1}, \ldots, \varphi_{n} \vdash \neg \neg \varphi$ and $\psi \vdash \psi$, we have that $\psi \subset_{2} \varphi_{1}, \ldots, \psi \subset_{2} \varphi_{n} \vdash \psi \subset_{2} \neg \neg \varphi$. But $\psi \subset_{2} \varphi_{1}, \ldots, \psi \subset_{2}$ $\varphi_{n} \in \Gamma^{\prime}$. So $\psi \subset_{2} \neg \neg \varphi \in \Gamma^{\prime}$ by application of Lemma 15 . Now, because we can easily prove that $\psi \subset_{2} \neg \neg \varphi \vdash \psi \subset_{2} \varphi$, we have that $\psi \subset_{2} \varphi \in \Gamma^{\prime}$, again by application of Lemma 15. This is impossible by assumption. Therefore, $S_{f}$ is $\mathrm{UL}^{*}$-consistent. Then, $S_{f}$ can be extended into a maximal $U L^{*}$-consistent set $\Gamma^{\prime \prime} \in \mathcal{M}^{c}$ by Lemma 16.

2. Now, let $S:=\{\psi\} \cup\left\{\neg \varphi \mid\right.$ there is $\varphi \notin \Gamma^{\prime \prime}$ such that $\left.\psi \subset_{2} \varphi \in \Gamma^{\prime}\right\}$. Assume that $S$ is not $\mathrm{UL}^{*}$-consistent. Then, there are $\neg \varphi_{1}, \ldots, \neg \varphi_{n} \in S$ such that $\psi, \neg \varphi_{1}, \ldots, \neg \varphi_{n} \vdash$. Then, $\psi \vdash \neg \neg \varphi_{1}, \ldots, \neg \neg \varphi_{n}$ by the rule $\neg R$. So, $\psi \vdash \neg \neg \varphi_{1} \vee \ldots \vee \neg \neg \varphi_{n}$ by iterated application of rule $\vee_{R}$ and $C_{R}$. Let $\varphi_{1}, \ldots, \varphi_{n}$ be the formulas of $\Gamma^{\prime \prime}$ associated to $\varphi_{1}, \ldots, \varphi_{n}$ through $S$. Then, because $\bigvee_{i} \varphi_{i} \vdash \bigvee_{i} \varphi_{i}$, we have that $\psi{ }_{,_{3}}\left(\neg \neg \varphi_{1} \vee \ldots \vee \neg \neg \varphi_{n}\right) \subset_{2}$ $\bigvee_{i} \varphi_{i} \vdash \bigvee_{i} \varphi_{i}$ by rule $\subset_{A}^{2}$. Therefore, $\left(\neg \neg \varphi_{1} \vee \ldots \vee \neg \neg \varphi_{n}\right) \subset_{2} \bigvee_{i} \varphi_{i} \vdash \psi \subset_{2} \bigvee_{i} \varphi_{i}(*)$ by rule $\subset_{K}^{2}$. Moreover, by Expression (19), it holds that $\neg \neg \varphi_{1} \subset_{2} \bigvee_{i} \varphi_{i}, \ldots, \neg \neg \varphi_{n} \subset_{2}$ $\bigvee_{i} \varphi_{i} \vdash\left(\neg \neg \varphi_{1} \vee \ldots \vee \neg \neg \varphi_{n}\right) \subset_{2} \bigvee_{i} \varphi_{i}(* *)$. However, we have that $\neg \neg \varphi_{i} \subset_{2} \bigvee_{i} \varphi_{i} \in$ $\stackrel{\imath}{\Gamma^{\prime}}$ for all $i$. Therefore, by application of Lemma 15 to $(* *)$, we have that $\left(\neg \neg \varphi_{1} \vee \ldots \vee \neg \neg \varphi_{n}\right) \subset_{2}$ $\bigvee_{i} \varphi_{i} \in \Gamma^{\prime}$. Then, by application of Lemma 15 to $(*)$, we have that $\psi \subset_{2} \bigvee_{i} \varphi_{i} \in \Gamma^{\prime}$. Thus, by definition of $S_{f}$, we have that $\bigvee_{i} \varphi_{i} \in S_{f}$. So, $\bigvee_{i} \varphi_{i} \in \Gamma^{\prime \prime}$. However, $\varphi_{i} \notin \Gamma^{\prime \prime}$ for all $i$. Therefore, $\bigwedge_{i} \neg \varphi_{i} \in \Gamma^{\prime \prime}$, contradicting $\bigvee_{i} \varphi_{i} \in \Gamma^{\prime \prime}$ (like in the previous case). Hence, we reach a contradiction. Therefore, $S$ is $\mathrm{UL}^{*}$-consistent. So, it can be extended to a maximal $\mathrm{UL}^{*}$-consistent set $\Gamma \in \mathcal{M}^{c}$ by Lemma 16 .

Now, we prove that we have $\left(\Gamma, \Gamma^{\prime}, \Gamma^{\prime \prime}\right) \in \mathcal{R}^{c}$. Let $\psi \subset_{2} \varphi \in \Gamma^{\prime}$ and assume towards a contradiction that $\varphi \in \Gamma$ but $\psi \notin \Gamma^{\prime \prime}$. Then, $\neg \varphi \in S$ by definition of $S$, so $\neg \varphi \in \Gamma$. This is impossible because $\varphi \in \Gamma$ and $\Gamma$ is a maximal $\mathrm{UL}^{*}$-consistent set. So, if $\varphi \in \Gamma$ then $\psi \in \Gamma^{\prime \prime}$. So, for all $\psi \subset_{2} \varphi \in \Gamma^{\prime}$, if $\varphi \in \Gamma$ then $\psi \in \Gamma^{\prime \prime}$. Hence, $\left(\Gamma, \Gamma^{\prime}, \Gamma^{\prime \prime}\right) \in \mathcal{R}^{c}$.

Moreover, $\varphi \in \Gamma$ and $\neg \psi \in \Gamma^{\prime \prime}$, so by Induction Hypothesis, $\mathcal{M}^{c}, \Gamma \Vdash \psi$ and not $\mathcal{M}^{c}, \Gamma^{\prime \prime} \Vdash \varphi$. Therefore, we do not have that $\mathcal{M}^{c}, \Gamma^{\prime} \Vdash \psi \subset_{2} \varphi$, which is impossible by assumption. So, finally, $\psi \subset_{2} \varphi \in \Gamma^{\prime}$.

- $\square_{j} \varphi$ and $\diamond_{j}^{-} \varphi$ :

The proof follows the same line as in [12], except that the structural negation $*$ has to be replaced by the logical negation $\neg$, similarly to $\otimes_{3}, \supset_{1}$ and $\subset_{2}$. 
Finally, we prove Theorem 4 .

Proof of Theorem 4 The proof of soundness is without difficulty, so we only prove strong completeness, i.e., we prove that for all sets $\Gamma \subseteq \mathcal{C}\left(\mathrm{C}_{\varphi}\right)^{*}$ and all $S:=X \vdash Y \in \mathcal{C}\left(\mathrm{C}_{\varphi}\right)^{*}$, if $\Gamma \vdash S$ holds then $\Gamma \vdash S$ is provable in $\mathrm{UL}^{*}$. We reason by contraposition. Assume towards a contradiction that it is not the case that $\Gamma \vdash S$. Then, it is not the case that there is a proof of $S$ in $\mathrm{UL}^{*}$ from $\Gamma$. Thus, it is not the case that $X, * Y \vdash$ can be proved in $\mathrm{UL}^{*}+\Gamma$. So, it is not the case that $\tau_{1}(X, * Y) \vdash$ can be proved in $\mathrm{UL}^{*}+\Gamma$, by the expressiveness results of [12]. That is, $E:=\left\{\tau_{1}(X, * Y)\right\}$ is $\mathrm{UL}^{*}+\Gamma$-consistent. So, by Lemma 16 (where $\mathrm{UL}^{*}$-consistency is replaced by $\mathrm{UL}^{*}+\Gamma$-consistency), it can be extended into a maximal $\mathrm{UL}^{*}+\Gamma$-consistent set $\Gamma^{\prime}$. Now, $\Gamma^{\prime}$ is also $U L^{*}$-consistent, so it is a possible world of $\mathcal{M}^{c}$ such that $E \cup \Gamma \subseteq \Gamma^{\prime}$. So, by Lemma 18 , for all $Z \in \Gamma^{\prime}$, we have that $\mathcal{M}^{c}, \Gamma^{\prime} \|^{A} Z$. Then, there is a pointed substructural model $\left(\mathcal{M}^{c}, \Gamma^{\prime}\right)$ such that $\mathcal{M}^{c}, \Gamma^{\prime} \|^{A} \Gamma$ and $\mathcal{M}^{c}, \Gamma^{\prime} \| \underline{A} \tau_{1}(X, * Y)$, i.e., such that $\mathcal{M}^{c}, \Gamma^{\prime} \|^{A} \Gamma$ and $\mathcal{M}^{c}, \Gamma^{\prime} \|^{A} X, * Y$ by the expressiveness results of [12]. That is, it is not the case that $\Gamma \Vdash S$, which contradicts our assumption. Thus, $\Gamma \vdash S$. 